\title{
The Non-Linear Power Spectrum of the Lyman Alpha Forest
}

\author{
Andreu Arinyo-i-Prats ${ }^{a}$, Jordi Miralda-Escudée ${ }^{b, a}$, Matteo Viel ${ }^{c, d}$ \\ and Renyue Cen ${ }^{e}$
}

\author{
${ }^{a}$ Institut de Ciències del Cosmos, Universitat de Barcelona, IEEC-UB, Barcelona 08028, \\ Catalonia. \\ ${ }^{b}$ Institució Catalana de Recerca i Estudis Avançats, Barcelona, Catalonia. \\ ${ }^{c}$ INAF, Astronomical Observatory of Trieste, 34131 Trieste, Italy \\ ${ }^{d}$ INFN, Sezione di Trieste, 34100 Trieste, Italy \\ ${ }^{e}$ Princeton University Observatory, Princeton, NJ 08544, USA \\ E-mail: andreuaprats@gmail.com , miralda@icc.ub.edu,viel@oats.inaf.it, \\ cen@astro.princeton.edu
}

\begin{abstract}
The Lyman alpha forest power spectrum has been measured on large scales by the BOSS survey in SDSS-III at $z \sim 2.3$, has been shown to agree well with linear theory predictions, and has provided the first measurement of Baryon Acoustic Oscillations at this redshift. However, the power at small scales, affected by non-linearities, has not been well examined so far. We present results from a variety of hydrodynamic simulations to predict the redshift space non-linear power spectrum of the Ly $\alpha$ transmission for several models, testing the dependence on resolution and box size. A new fitting formula is introduced to facilitate the comparison of our simulation results with observations and other simulations. The non-linear power spectrum has a generic shape determined by a transition scale from linear to non-linear anisotropy, and a Jeans scale below which the power drops rapidly. In addition, we predict the two linear bias factors of the Ly $\alpha$ forest and provide a better physical interpretation of their values and redshift evolution. The dependence of these bias factors and the non-linear power on the amplitude and slope of the primordial fluctuations power spectrum, the temperature-density relation of the intergalactic medium, and the mean Ly $\alpha$ transmission, as well as the redshift evolution, is investigated and discussed in detail. A preliminary comparison to the observations shows that the predicted redshift distortion parameter is in good agreement with the recent determination of Blomqvist et al., but the density bias factor is lower than observed. We make all our results publicly available in the form of tables of the non-linear power spectrum that is directly obtained from all our simulations, and parameters of our fitting formula.
\end{abstract}

Keywords: Cosmology, intergalactic medium, cosmological simulations, quasars: absorption spectra 


\section{Contents}

1 Introduction $\quad 1$

2 The Bias Factors of the Lyman Alpha Forest 4

3 Method of Analysis of the Simulations $\quad 7$

$\begin{array}{lll}3.1 & \text { Simulation characteristics } & 7\end{array}$

3.1.1 SPH simulations 8

3.1.2 Eulerian simulation 9

3.2 Extracting the Ly $\alpha$ power spectrum from the simulations 10

3.3 Power spectrum estimation, Fourier space binning, and errorbars 12

3.4 Parameterized fitting function for the $\operatorname{Ly} \alpha$ power spectrum 14

4 Results: the L120 Simulation $\quad \mathbf{1 5}$

$\begin{array}{lll}4.1 & \text { Results for the linear bias factors } & 18\end{array}$

4.2 The non-linear part of the power spectrum 21

5 Convergence Tests: Resolution, Box Size and Numerical Method 22

5.1 Resolution 23

5.2 Simulation Variance and Box Size 27

5.3 Comparing Lagrangian and Eulerian simulations 31

6 Dependence of the Ly $\alpha$ Power Spectrum on the Physical Model 34

6.1 Power spectrum amplitude 35

$\begin{array}{lll}6.2 & \text { Power spectrum slope } & 37\end{array}$

6.3 Mean transmission fraction and redshift evolution 39

6.4 Temperature-Density relation 41

7 Discussion $\quad 45$

$\begin{array}{lll}7.1 & \text { Comparison to observations } & 48\end{array}$

8 Conclusions $\quad 49$

Appendix A Dependence of the Bias Factors on the Minimum Error param-

eter $\quad 53$

Appendix B Tables $\quad 55$

\section{Introduction}

The Ly $\alpha$ forest is the main observational probe for studying the structure and evolution of the intergalactic medium (IGM). The transmitted Ly $\alpha$ flux observed in the spectrum of a distant source (typically a quasar) provides us with a one-dimensional map of absorption along the line of sight (LOS), with the observed wavelength corresponding to the redshift of the intervening neutral hydrogen causing the Ly $\alpha$ scattering (e.g. [43, 49]). 
The way in which the flux transmission fraction is related to the underlying density field, temperature and peculiar velocity gradient of the gas on small scales is non-linear, and can be modelled in detail only from hydrodynamic cosmological simulations $[12,16,27,35$, $39,44,55,58,60]$. However, in the limit of large scales, the transmission fraction averaged over a large region depends linearly on the mean overdensity and peculiar velocity gradient in the region, and the power spectrum of the transmission is simply proportional to the power spectrum of mass fluctuations, with the standard redshift distortions that were predicted initially for galaxy surveys $[25,29]$.

This simple linear treatment of the Ly $\alpha$ forest applicable on large scales has led to its use as a cosmological tool to measure the power spectrum, first from few tens of quasar spectra [where only the projected one-dimensional power as a function of the parallel Fourier component is measured; see 16, 17, 39, 41, 58] and then in full redshift space, where the correlation in the transmission among parallel lines of sight is used [52]. As proposed in [38], and implemented in the Baryon Oscillation Spectroscopic Survey [BOSS; see 18] of the Sloan Digital Sky Survey-III [SDSS-III, see 20], the Ly $\alpha$ power spectrum is proving to be a powerful tool to measure the general large-scale matter power spectrum, and in particular to measure the baryon acoustic oscillation scale, at a relatively high redshift which has not so far been probed by other observables. This measurement provides geometrical constraints on the expansion rate and the angular diameter distance as a function of redshift $[8,19,22,53]$.

In contrast to the large scales, the Ly $\alpha$ power spectrum at small scales is affected by a variety of non-linear physical processes governing the evolution of the IGM. These physical processes are highly complex, and they may include several phenomena related to the formation of stars and quasars in galaxies that can perturb the IGM. Some examples are reionization and the inhomogeneous heating caused by it, and the hydrodynamic effects from galactic winds and quasar jets. There is, however, a more simple assumption that can be made for the evolution of the IGM: that the ionization of the IGM is caused only by a nearly uniform radiation background, which produces a nearly uniform heating, and that shock waves arise only from the gravitational collapse of structure formation and not from the ejection of any gas from galaxies due to supernovae-driven winds or quasars. Even though it is known that quasar jets and galaxy winds are present in the universe and they have some impact on the IGM, the volume they affect may in practice be very small $[40,56,59]$, and it is useful to test first the most simple assumption for the evolution of the IGM against the observations. This simple model should be mostly described by only five parameters, which determine the statistical properties of the $\operatorname{Ly} \alpha$ forest:

- The mean transmission fraction, $\bar{F}(z)$, which depends on the intensity of the cosmic ionizing background and is directly measured in the observations (except for uncertainties related to continuum fitting).

- The density-temperature relation, usually parameterized by the two parameters $T_{0}$ and $\gamma$ in the power-law relation $T=T_{0}(1+\delta)^{\gamma}$, where $\delta=\rho / \bar{\rho}-1$ is the gas overdensity. When the IGM is heated in photoionization equilibrium and cools adiabatically due to Hubble expansion, one expects this power-law relation to hold with $\gamma \simeq 0.6$, but the relation may be altered by the heating due to HeII reionization [28, 42, 45].

- The mass power spectrum of primordial perturbations near the Jeans scale of the IGM, $\lambda_{J}=2 \pi / k_{J}$, which we can parameterize also with two parameters as a power-law with free amplitude and index, $P(k)=A_{\alpha}\left(k / k_{J}\right)^{n_{\alpha}}$. The characteristic Jeans scale at the 
mean density is related to the IGM temperature, although in detail it depends also on the entire thermal history [23], and therefore may be considered as a sixth parameter.

Even though the large-scale properties of the Ly $\alpha$ forest are simply understood from linear theory, there is a strong interest in understanding the small-scale, non-linear properties as well. There are several motivations for this: first, we need to test if our understanding of the IGM in terms of a simple uniform photoionization as mentioned above is essentially correct, or if there are important modifications due to a strong impact of galactic winds and jets [e.g., 32], or large inhomogeneities due to HeII reionization [15, 42]. Second, the Ly $\alpha$ forest linear power spectrum depends on two bias factors, with values that can be measured and can be predicted from an understanding of the small-scale physics. Finally, the Ly $\alpha$ power spectrum transmission power spectrum is being measured to increasingly high accuracy, both in projection [from single lines of sight, see 41, 46], and in its full shape in redshift space [3], and a detailed comparison of the observations with predictions from numerical simulations of the fully non-linear power spectrum may offer us new clues to essential questions in cosmology, such as the impact of neutrino masses on the growth of structure [47], or limits on models of warm dark matter [57], or other possible variations on the nature of the dark matter. There is therefore a need to obtain reliable theoretical predictions for the non-linear power spectrum of the Ly $\alpha$ transmission fraction as a function of redshift from numerical simulations of a large array of cosmological models, in terms of the most important Ly $\alpha$ forest parameters mentioned above.

The goal for the theory of the non-linear Ly $\alpha$ forest is comparable to that of numerical simulations of the hot, X-ray emitting gas in clusters of galaxies. Detailed determinations of the gas density and temperature distributions from X-ray observations and the Sunyaev-Zeldovich effect, together with the mass distribution from gravitational lensing and the kinematic distribution of galaxies, have spurred advances in the theoretical modelling of clusters, the comparison of numerical codes for cosmological simulations, and tests of the convergence of the results. At present, the abundance of clusters of galaxies can be used to infer the normalization of the mass power spectrum, but this determination depends on the uncertain relation between the observable properties from X-rays, gravitational lensing and the Sunyaev-Zeldovich temperature decrement to the cluster mass. This relation needs to be predicted from numerical simulations, and the theoretical modelling affects the comparison with the power spectrum normalization derived from CMB observations [e.g., 26]. Similarly, the Ly $\alpha$ forest is sensitive to the amplitude of the power spectrum and several other cosmological parameters and physical properties of the IGM, but constraints on these quantities can only be inferred once we have a reliable understanding and modelling of non-linear effects on the observed properties of the $\operatorname{Ly} \alpha$ forest.

The aim of this work is to study several cosmological simulations of the Ly $\alpha$ forest for a variety of models, to analyze the non-linear power spectrum of the Ly $\alpha$ transmission that they predict, and to test the conditions that the simulations must satisfy, in terms of resolution and simulation volume, to reach convergence of the results. This problem was first addressed in the pioneering paper of [36] (hereafter M03), and here we attempt to continue this study by examining a large number of hydrodynamic simulations, characterizing the power spectrum with a new, simpler fitting formula with several non-linear parameters, and studying the dependence of the linear bias factors on the IGM properties. We start in $\S 2$ by reviewing the definition of the linear bias factors of the Ly $\alpha$ forest and the derivation of the linear power spectrum, and we introduce alternative bias factors for the Ly $\alpha$ effective optical depth. The simulations and our technique for measuring and fitting the power spectrum are 
explained in $\S 3$. The results for the non-linear power spectrum in one specific model, for which we have run our largest simulation, are presented in $\S 4$. Several tests of convergence with the box size and resolution of the simulations are performed in $\S 5$, and the results for the power spectrum fits for a variety of different physical models are presented in $\S 6$. Finally, the results are discussed in $\S 7$ and conclusions are given in $\S 8$.

\section{The Bias Factors of the Lyman Alpha Forest}

Before proceeding to describe our analysis of hydrodynamic simulations, we review here the standard definitions of the power spectrum and bias factors of the Ly $\alpha$ forest. We also introduce a new definition for the optical depth bias factors, already discussed in the context of metal lines in [21], which is useful to better interpret their values and compare results at different redshifts and for different types of absorption systems.

The transmission fraction $F$ in the Ly $\alpha$ forest is the ratio of the observed flux $f$ that is transmitted from the source to the continuum flux, $f_{c}$, when there is no intervening absorption. Usually a model of the source continuum spectrum is used to calculate $F=f / f_{c}$ from the observed flux. The fluctuation in the transmission is defined as

$$
\delta_{F}=\frac{F}{\bar{F}(z)}-1
$$

where $\bar{F}(z)$ is the mean value of the transmission, evaluated as a function of redshift. The power spectrum $P_{F}(k, \mu)$ is that of $\delta_{F}$ in redshift space, with $\mu$ being the cosine of the angle of the Fourier wavevector of modulus $k$ from the LOS to the observed source. On small scales, the value of $\delta_{F}$ at a certain point depends on the complex non-linear evolution of the IGM. However, the average of $\delta_{F}$ over a large enough scale is related to the mass density fluctuations according to a linear expression of the form $\delta_{F}=b_{F \delta} \delta+b_{F \eta} \eta$. Here, $\delta$ is the mass density fluctuation, and

$$
\eta=-\frac{1}{a H} \frac{\partial v_{p}}{\partial x_{p}}
$$

is the dimensionless gradient of the peculiar velocity $v_{p}$ along the LOS, both averaged over the same large region that is used to average $\delta_{F} ; x_{p}$ is the comoving LOS coordinate, $H$ is the Hubble constant, and $a$ the scale factor. This is the most general linear expression for any tracer of large-scale structure. The reason is that the only scalar quantities that can be constructed from the deformation tensor, $\phi_{, i j}$ (equal to the second derivatives of the gravitational potential), and the LOS unit vector, $n_{i}$, are the trace of $\phi$, which is proportional to $\delta$, and $n_{i} n_{j} \phi_{, i j}$, which is proportional to $\eta$. The bias factors are simply defined as the partial derivatives of $\delta_{F}$ with respect to $\delta$ and $\eta$, after the large-scale averaging is done:

$$
b_{F \delta}=\frac{\partial \delta_{F}}{\partial \delta}, \quad b_{F \eta}=\frac{\partial \delta_{F}}{\partial \eta},
$$

where each partial derivative is understood to be done by holding the other variable $(\delta$ or $\eta)$ constant.

The bias factors in equation (2.3) are not defined in the same way as for point objects like galaxies. In general, denser regions or regions where the Hubble expansion rate has slowed down have stronger absorption, and therefore lower transmission $F$, so the values of $b_{F \delta}$ and $b_{F \eta}$ are negative. Moreover, when $\bar{F}$ is close to unity (which occurs at low redshift), the absolute values of the bias factors are very small simply because they express the absorption 
fluctuation compared to the total transmitted fraction, when the mean absorption is very small. A physical bias factor should reflect the fluctuation of a quantity that is zero when the mass density is zero, whereas the transmitted fraction is obviously one when the gas density is zero. The quantity that reflects the relative amount by which the Ly $\alpha$ absorption fluctuates when the mass or the peculiar velocity gradient fluctuate is obtained from the fluctuation of an effective optical depth, $\tau_{e}=-\log F$ (where $F$ has been averaged over a large, linear scale before taking the logarithm), which has a relative fluctuation $\delta_{\tau}=\delta_{F} / \log \bar{F}=-\delta_{F} / \bar{\tau}_{e}$. The bias factors for this effective optical depth fluctuation are:

$$
b_{\tau \delta}=\frac{\partial \delta_{\tau}}{\partial \delta}=\frac{b_{F \delta}}{\log \bar{F}}, \quad b_{\tau \eta}=\frac{\partial \delta_{\tau}}{\partial \eta}=\frac{b_{F \eta}}{\log \bar{F}} .
$$

The usefulness of these new definitions is made apparent by considering various simple models for the Ly $\alpha$ forest. Let us first imagine that the Ly $\alpha$ absorption systems are clouds of gas distributed in space with a density bias factor $b_{c}$; in other words, wherever the mass density fluctuates by $\delta$, the number density of gas clouds fluctuates by $b_{c} \delta$, but their individual absorption line profiles do not vary. If the internal dynamics of these clouds are not aligned in a correlated way with the LOS depending on the value of $\eta$, the effective optical depth they produce in the spectrum fluctuates as $\delta_{\tau}=b_{c} \delta+\eta$, because a peculiar velocity gradient is simply squeezing the absorption lines of the clouds in the spectrum by the factor $1-\eta$, without altering the absorption line profiles. Hence, for these population of clouds we have $b_{\tau \delta}=b_{c}$ and $b_{\tau \eta}=1$ : the density bias factor reflects the true physical bias of the population of clouds, and the peculiar velocity gradient bias factor is equal to one, just like for any population of objects that is selected isotropically, i.e., independently of the LOS direction.

Next, consider the case where the IGM absorption is optically thin everywhere. The optical depth is proportional to the density of hydrogen atoms, which on large scales must again behave like a biased population of objects with some bias factor $b_{a}$, and $b_{\tau \eta}$ must again be unity because the column density of hydrogen atoms in a given interval of the Ly $\alpha$ spectrum is proportional to the integrated optical depth, which cannot be modified by any shifts due to peculiar velocities.

Therefore, if we consider that the true $\operatorname{Ly} \alpha$ forest is a combination of optically thin absorption by the IGM, plus a population of clouds with optically thick absorption lines that follow a linear bias factor but do not change their internal properties as a function of $\delta$ and do not have correlated orientations with the principal axes of the large-scale deformation tensor, we conclude that $b_{\tau \eta}=1$, and $b_{\tau \delta}$ reflects a true, physical bias factor that results from a weighted average of the optically thin, intergalactic neutral hydrogen bias $b_{a}$, and the gas clouds bias $b_{c}$.

This model is of course not exactly correct, because there is an intermediate density range of gas that is not optically thin and is not in a population of clouds that have lost any alignment of their internal dynamics with the surrounding large-scale structure. Nevertheless we can expect it to provide a first approximation to the reality of the Ly $\alpha$ forest, and in this way the value of the two bias factors can have a physical interpretation. Note also that these optical depth bias factors can be defined for any other set of Lyman series lines and metal lines, simply by using the appropriate value of $\bar{F}$ in equation (3.1), as proposed in [21], and then the bias factors of any hydrogen or metal absorption lines associated with a certain population of clouds or galaxies are equal to the true bias factors of these objects.

The linear power spectrum is derived as in [29], from the simple fact that in the linear regime, $\eta=f(\Omega) \mu^{2} \delta$ in Fourier space, where $f(\Omega)=d \log G / d \log a$, and $G(a)$ is the growth 
factor. For the linear power spectrum of the transmission fluctuation $\delta_{F}$, we have

$$
P_{F}(k, \mu)=b_{F \delta}^{2}\left(1+\beta \mu^{2}\right)^{2} P_{L}(k)+N_{0},
$$

where the redshift distortion parameter is

$$
\beta=\frac{b_{F \eta} f(\Omega)}{b_{F \delta}},
$$

and $P_{L}$ is the matter density fluctuation power spectrum. We have included the intrinsic shot noise term $N_{0}$, which is a shot noise that should remain even when $P_{F}$ is measured from an arbitrarily dense set of absorption sightlines due to the random nature of the formation of absorption line systems as the evolution of the IGM becomes non-linear [37], although this is believed to be very small and we shall ignore it in this paper. This linear power spectrum is of course valid only in the limit of large scales, and the models we shall use to fit our simulations include a non-linear multiplicative term, as described below in equation (3.3. The linear power spectrum of the effective optical depth $\delta_{\tau}$ is the same, except for the normalization, which changes by replacing $b_{F \delta}$ by $b_{\tau \delta}$. Note that $\beta$ is the same for the transmission or effective optical depth power spectra, because the ratio of the two bias factors remains unaltered.

Finally, we mention also the radiation bias factor $b_{F \Gamma}$, defined as the variation of $\delta_{F}$ when the photoionization rate of hydrogen $\Gamma$, determined by the intensity of the ionizing background, varies. If $\delta_{\Gamma}$ is the relative fluctuation of this photoionization rate, then the total transmission fluctuation averaged on a large scale is $\delta_{F}=b_{F \delta} \delta+b_{F \eta} \eta+b_{F \Gamma} \delta_{\Gamma}$. The modification of the power spectrum $P_{F}$ due to fluctuations in the ionizing radiation intensity caused by sources that are tracers of the mass density fluctuations was discussed in [24, 48]. We ignore here the possible additional effect from HeII reionization of a large-scale modification of the temperature-density relation. Under the assumption that the intensity of the ionizing background does not appreciably affect the temperature and hydrodynamic evolution of the IGM, and changes the optical depth at every spectral pixel in inverse proportion to the photoionization rate, then the variation of the transmission fluctuation with $\delta_{\Gamma}$ can be computed in terms of the probability distribution of the transmission fraction $P(F)$, as

$$
\delta_{F}=\frac{1}{\bar{F}} \int_{0}^{1} d F P(F) \exp \left(\frac{\log F}{1+\delta_{\Gamma}}\right)-1=-\frac{\int_{0}^{1} d F P(F) F \log F}{\bar{F}} \delta_{\Gamma}=b_{F \Gamma} \delta_{\Gamma} .
$$

Just as before, we define the optical depth radiation bias factor as

$$
b_{\tau \Gamma}=\frac{b_{F \Gamma}}{\log \bar{F}}=-\frac{\int_{0}^{1} d F P(F) F \log F}{\bar{F} \log \bar{F}} .
$$

An alternative simple model for computing the peculiar velocity gradient bias factor is to assume that all the fluctuations determining the Ly $\alpha$ forest absorption spectrum can be treated in the linear regime. Using this assumption, [51] showed that $b_{F \eta}$ should be given by the same expression for $b_{F \Gamma}$ in equation (2.8). The reason is easy to understand: for linear fluctuations, the optical depth at any spectral pixel is simply multiplied by the factor $(1-\eta)^{-1} \simeq 1+\eta$ under the effect of a peculiar velocity gradient, so the same derivation shows that $b_{F \eta}=b_{F \Gamma}$. For the case of radiation fluctuations, however, the derivation of equation (2.8) does not need to assume that the Ly $\alpha$ forest fluctuations are linear even on small scales, 
so the prediction for the radiation bias factor is much more reliable. We will show in $\S 4$ that in fact, $b_{F \eta}$ is quite different from $b_{F \Gamma}$ because of the non-linearities that affect the change of small scale Ly $\alpha$ forest fluctuations under a variation of the large-scale peculiar velocity gradient.

\section{Method of Analysis of the Simulations}

Our goal in this paper is to use cosmological hydrodynamic simulations of the IGM to predict the three-dimensional power spectrum of the Lyman alpha forest in redshift space, $P_{F}(k, \mu ; z)$, where $k$ and $\mu$ are the modulus and the cosine of the angle from the LOS of the Fourier mode vector, and $z$ is the redshift. The simulations used are described in $\S 3.1$. The method of analysis is inspired in that of M03 and is based on the following steps: (1) starting from a grid of cells containing the hydrodynamic quantities of gas density, ionized fraction, temperature and velocity at a certain redshift output of a simulation, the corresponding spectra of Ly $\alpha$ transmission are computed for the entire grid, using one of the simulation axes as the assumed LOS direction, and the three-dimensional Fast Fourier Transform of this transmission field is obtained, as described in $\S 3.2 ;(2)$ the mean value of $P_{F}(k, \mu)$ is computed in bins of $(k, \mu)$, and errorbars are assigned which take into account the variance due to the finite simulation volume (§3.3); (3) a parameterized fitting function for $P_{F}(k, \mu)$ is chosen to obtain best-fit values of the parameters for several simulations (§3.4).

\subsection{Simulation characteristics}

Two types of hydrodynamic simulations will be used in this chapter. Most of the simulations rely on the Tree-PM (Particle Mesh) Smoothed Particle Hydrodynamics (SPH) GADGETII code [54], and the bulk of our analysis will be performed on the outputs of these Lagrangian simulations. One simulation that is based on a fixed-grid Eulerian code is also used, to allow for a first comparison of the results for the two types of hydrodynamic numerical methods. This simulation is described in [11].

Table 3.1 shows a list of all the simulations that will be used in this work, including variations in the spatial grid size and the spectral pixel size for the analysis of the Ly $\alpha$ forest. The first two columns give the comoving box size, $L$, and the number of dark matter particles in the SPH simulations (the number of dark matter and gas particles in the SPH simulations being the same). The third one gives the number of cells, $N_{c}$, in the uniform grid that is constructed to compute the density, temperature and velocity in real space. Note that the simulation labelled Euler does not use particles, and the cells used to run the simulation are $N_{c}^{3}$ and are directly used as this spatial grid. The Ly $\alpha$ spectra are computed for each of the three axes of the simulation playing the role of the LOS, with the number of pixels in each spectrum from each row of $N_{c}$ cells of length $L$ given in the fourth column; generally there are as many pixels in the spectra as cells in the spatial grid, except in the analysis labelled P1024 where the number of pixels is doubled. The other columns give values of physical parameters used in the simulations: the variable $\sigma_{8}$ parameterizing the present amplitude of linear perturbations on a sphere of $8 h^{-1} \mathrm{Mpc}$, the mean temperature at the mean density $T_{0}$, and the power-law index that fits the density-temperature relation at low densities, which is described below in more detail. In general, models have variations of different parameters around the values of the fiducial model in the first row of Table 3.1, and they are labelled with names that refer to the parameter that is being varied. 


\begin{tabular}{|c|cccc|ccc|}
\hline Name & Box size & Particles & $N_{c}$ & Pixels & $\sigma_{8}$ & $\gamma$ & $\log \left(T_{0}\right)$ \\
\hline \hline Fiducial & $60 \mathrm{Mpc} / \mathrm{h}$ & $512^{3}$ & $512^{3}$ & 512 & 0.8778 & 1.6 & 4.3 \\
P1024 & $60 \mathrm{Mpc} / \mathrm{h}$ & $512^{3}$ & $512^{3}$ & 1024 & 0.8778 & 1.6 & 4.3 \\
C256 & $60 \mathrm{Mpc} / \mathrm{h}$ & $256^{3}$ & $256^{3}$ & 256 & 0.8778 & 1.6 & 4.3 \\
R384 & $60 \mathrm{Mpc} / \mathrm{h}$ & $384^{3}$ & $512^{3}$ & 512 & 0.8778 & 1.6 & 4.3 \\
R384C & $60 \mathrm{Mpc} / \mathrm{h}$ & $384^{3}$ & $256^{3}$ & 256 & 0.8778 & 1.6 & 4.3 \\
R640 & $60 \mathrm{Mpc} / \mathrm{h}$ & $640^{3}$ & $512^{3}$ & 512 & 0.8778 & 1.6 & 4.3 \\
R640C & $60 \mathrm{Mpc} / \mathrm{h}$ & $640^{3}$ & $256^{3}$ & 256 & 0.8778 & 1.6 & 4.3 \\
L80 & $80 \mathrm{Mpc} / \mathrm{h}$ & $512^{3}$ & $512^{3}$ & 512 & 0.8778 & 1.6 & 4.3 \\
L120 & $120 \mathrm{Mpc} / \mathrm{h}$ & $768^{3}$ & $512^{3}$ & 512 & 0.8778 & 1.6 & 4.3 \\
\hline Euler & $50 \mathrm{Mpc} / \mathrm{h}$ & - & $2048^{3}$ & 2048 & 0.82 & 1.54 & 4.03 \\
Lagrange & $50 \mathrm{Mpc} / \mathrm{h}$ & $512^{3}$ & $512^{3}$ & 512 & 0.82 & 1.58 & 4.10 \\
Planck & $60 \mathrm{Mpc} / \mathrm{h}$ & $512^{3}$ & $512^{3}$ & 512 & 0.8338 & 1.4 & 4.2 \\
\hline G1.3 & $60 \mathrm{Mpc} / \mathrm{h}$ & $512^{3}$ & $512^{3}$ & 512 & 0.8778 & 1.3 & 4.3 \\
G1.0 & $60 \mathrm{Mpc} / \mathrm{h}$ & $512^{3}$ & $512^{3}$ & 512 & 0.8778 & 1.0 & 4.3 \\
G1T4 & $60 \mathrm{Mpc} / \mathrm{h}$ & $512^{3}$ & $512^{3}$ & 512 & 0.8778 & 1.0 & 4.0 \\
S0.76 & $60 \mathrm{Mpc} / \mathrm{h}$ & $512^{3}$ & $512^{3}$ & 512 & 0.7581 & 1.6 & 4.3 \\
S0.64 & $60 \mathrm{Mpc} / \mathrm{h}$ & $512^{3}$ & $512^{3}$ & 512 & 0.6396 & 1.6 & 4.3 \\
\hline
\end{tabular}

Table 1. List of simulations and analysis variations used in this paper. The first column lists the name we give to the simulation/analysis, the second the simulation box size, and the third indicates the number of particles used in the simulation (both for dark matter and gas, except for the simulation named Euler, which uses a fixed Eulerian grid instead of gas particles). The fourth column gives the number of cells, $N_{c}$, used to represent the hydrodynamic variables in the spatial grid that is computed to obtain the Ly $\alpha$ forest spectra, and the fifth column is the number of pixels on the line of sight direction used to compute the Ly $\alpha$ spectra. The last three columns give the power spectrum amplitude and the $\left(T_{0}, \gamma\right)$ parameters of the temperature-density relation in the simulation, where $T_{0}$ is expressed in kelvin.

\subsubsection{SPH simulations}

All simulations in Table 3.1 except for the one denoted as Euler were run using the publicly available Tree-Particle Mesh Smoothed Particle Hydrodynamics (SPH) GADGET-II code [54].

The fiducial simulation uses a box of 60 comoving $h^{-1} \mathrm{Mpc}$ and $2 \times 512^{3}$ particles (for the total of gas and dark matter). Other simulations are run with larger boxes of 80 and 120 $h^{-1} \mathrm{Mpc}$ (L80 and L120) to test the effect of the missing large-scale power, or with different resolution to check the convergence as the particle masses are reduced. The cosmological model is flat $\Lambda \mathrm{CDM}$ with the following parameters, using standard notation: $\Omega_{0 \mathrm{~m}}=0.3$, $\Lambda=0.7, \Omega_{0 \mathrm{~b}}=0.05, H_{0}=70 \mathrm{~km} \mathrm{~s}^{-1} \mathrm{Mpc}^{-1}, n_{\mathrm{s}}=1$ and $\sigma_{8}=0.8778$. The initial conditions are generated using the software $\mathrm{CAMB}^{1}$ and the Zel'dovich approximation at the initial redshift of $z=49$. The particle mesh grid used to calculate the long range forces is chosen to have the same number of cells as the number of gas particles, $512^{3}$, while the gravitational softening is $4 \mathrm{kpc} / h$ in comoving units for the $60 h^{-1} \mathrm{Mpc}$ box and scales proportionally to the initial particle separation for the other simulations. The hydrodynamical processes are followed according to the prescription of [30]. Star formation is also included in the model with a simplified prescription that allows to instantaneously convert into a star particle any

\footnotetext{
${ }^{1}$ http://camb.info/readme.html
} 
gas particle of overdensity larger than 1000 and temperature colder than $10^{5} \mathrm{~K}$. This has been demonstrated to have negligible impact on the Ly $\alpha$ forest transmission power spectrum [58].

Most of the SPH simulations in Table 3.1 are based on the same cosmological model as the fiducial one. The exceptions are the simulations S0.76 and S0.64, where the amplitude of the initial power spectrum is varied, and the Planck and Lagrange simulations. The Planck simulation uses a model that is consistent with the most recent CMB measurements from the Planck mission (REFERENCE), with the following parameters: $\Omega_{0 \mathrm{~m}}=0.3175, \Lambda=0.6825$, $\Omega_{0 \mathrm{~b}}=0.049, H_{0}=67.11 \mathrm{~km} \mathrm{~s}^{-1} \mathrm{Mpc}^{-1}, n_{\mathrm{s}}=0.9624$ and $\sigma_{8}=0.8338$. The Lagrange simulation is run for the same cosmological model as the Euler simulation that is run with the Eulerian code, described below.

Our main goal in analyzing these simulations is to understand how the non-linear power spectrum varies several physical parameters, such as the amplitude $\sigma_{8}$ or the densitytemperature relation. These comparison are limited by the intrinsic random variations in the measured power due to the sample variance in simulations of limited box size. To reduce this intrinsic sample variance, we have chosen to generate the initial conditions of all the GADGET-II simulations by setting the amplitude of every Fourier mode to the exact rms amplitude predicted by the power spectrum, instead of generating it following the Rayleigh distribution. The mode phases are still generated randomly. Only for the fiducial simulation, we have generated several realizations with different random seeds, including some cases where the Rayleigh distribution for the amplitudes is included, which will be analyzed in $\S 5.2$ to check for any effect that this can have on our results. This implies that the power spectrum of the initial conditions for our simulations is exactly equal to the value predicted by the cosmological model at each Fourier mode, without any variations due to sample variance. The Ly $\alpha$ transmission power spectrum that is obtained, however, has random variations caused by non-linear Fourier mode couplings.

The impact of different thermal histories on the Ly $\alpha$ forest is explored by modifying the Ultra Violet (UV) background photo-heating rate in the simulations, as in [6]. A power-law temperature-density relation, $T=T_{0}(1+\delta)^{\gamma-1}$, arises in the low density IGM as a natural consequence of the interplay between photo-heating and adiabatic cooling [28]. Two different values for the temperature at mean density, $T_{0}$, and three different values for the power-law index of the temperature-density relation, $\gamma$, are considered to examine the impact of the temperature-density relation on the $\operatorname{Ly} \alpha$ power spectrum; the most recent observational constraints [4] favor values for these parameters close to those of our fiducial model. The different thermal histories are constructed by modifying the fiducial simulation He II photoheating rate according to $\epsilon_{H e I I}=\alpha \times \epsilon_{f i d, H e I I}^{\nu}$, changing the parameters $\alpha$ and $\nu$ [6]. The fiducial thermal history appears to be in overall good agreement with recent determinations based on line profile fitting [5]. The thermal histories for the SPH runs are built to guarantee that the $\rho-T$ relation is approximately constant with redshift in the range $z=2.2-3$, so the quoted values of $\gamma$ and $T_{0}$ approximate reasonably well the density-temperature relation in the whole redshift range investigated here.

\subsubsection{Eulerian simulation}

In addition to the SPH simulations based on a Lagrangian approach, a simulation based on an Eulerian code is used, [described in $9,10,13,14]$. The simulation used here is on a 50 $\mathrm{Mpc} / \mathrm{h}$ box with $2048^{3}$ cells, and uses the cosmological model with parameters $\Omega_{0 \mathrm{~m}}=0.28$, $\Omega_{0 \mathrm{~b}}=0.04, \Lambda=0.72, H_{0}=70 \mathrm{~km} \mathrm{~s}^{-1} \mathrm{Mpc}^{-1}, n_{s}=0.96$, and $\sigma_{8}=0.82$. This simulation has 
been run with standard initial conditions, with the amplitudes of every Fourier mode following the Rayleigh distribution. The $\rho-T$ relation is determined by following the photoionization heating that is derived from a model of the ionizing background that is computed as the simulation is run. In the density range that is important for the Ly $\alpha$ forest over the redshift range $2.2<z<3$ that we analyze in this paper, this $\rho-T$ relation is well approximated by the values of $T_{0}$ and $\gamma$ in Table 3.1.

To compare this simulation with an equivalent one run with the SPH method, we have run the simulation designated "Lagrange" in Table 3.1. The Lagrange simulation is run for the same cosmological model and box size as the Euler one, and using HeII heating parameters to approximately mimick the $\rho$-T relation in the Euler one. This is further discussed in $\S 5.3$.

\subsection{Extracting the $\operatorname{Ly} \alpha$ power spectrum from the simulations}

We now start discussing the full procedure for processing the simulation outputs to obtain an estimate of the Ly $\alpha$ transmission power spectrum, and for analyzing fits to this power spectrum. This procedure is summarized in the diagram in figure 1, and discussed in detail in the rest of this section.

For the GADGET-II simulations, the SPH formalism for computing the hydrodynamic variables of gas density, temperature and velocity on a Cartesian grid, and then extracting mock Ly $\alpha$ spectra, is followed as described in the Appendix A4 of [55]. For the Euler simulation, the Cartesian grid that the simulation is run on is used directly to obtain the Ly $\alpha$ spectra, as in [44]. Each simulation grid in real space is used to generate three distinct boxes of Ly $\alpha$ spectra, taking each of the three axes as the LOS. For each of the three axes, the spectra for the entire simulated box are computed, resulting in $N_{c}^{2} \operatorname{Ly} \alpha$ spectra.

Apart from the parameters of each simulation, an additional parameter is necessary to compute the $\operatorname{Ly} \alpha$ spectra: the intensity of the ionizing background, which can be altered to adjust the mean transmission $\bar{F}(z)$ to a certain value. The mean transmission fraction is fixed to the value given by the expression

$$
\bar{F}(z)=\exp \left[-0.0023(1+z)^{3.65}\right],
$$

which was found to adequately fit the observational data of high-resolution spectra by [31], after subtracting the estimated metal contribution. We note that more recent determinations give comparable values of $\bar{F}$, but there are substantial uncertainties in this determination $[1,4]$.

The computed Ly $\alpha$ spectra are modified to adjust this value of the mean transmission by using the approximation that the optical depth varies at each pixel as the inverse of the intensity of the ionizing background, and that the gas temperature is not affected by this background intensity. This assumes that collisional ionization can be neglected and that the atomic fraction is much smaller than unity, which is generally an excellent approximation (except in high density regions where the optical depth is very large in any case, and therefore does not affect the computed Ly $\alpha$ spectra). The use of this approximation avoids having to recompute the Ly $\alpha$ spectra every time that the mean transmission is adjusted to the required value in the expression above. The assumption that the temperature and hydrodynamic evolution of the IGM is not affected by the intensity of the ionizing background is not as accurate because cooling by line excitation is neglected, but in any case, here we are interested in examining the dependence of the predictions for $P_{F}$ on an assumed, fixed $\rho$-T relation, and separately on $\bar{F}$. 


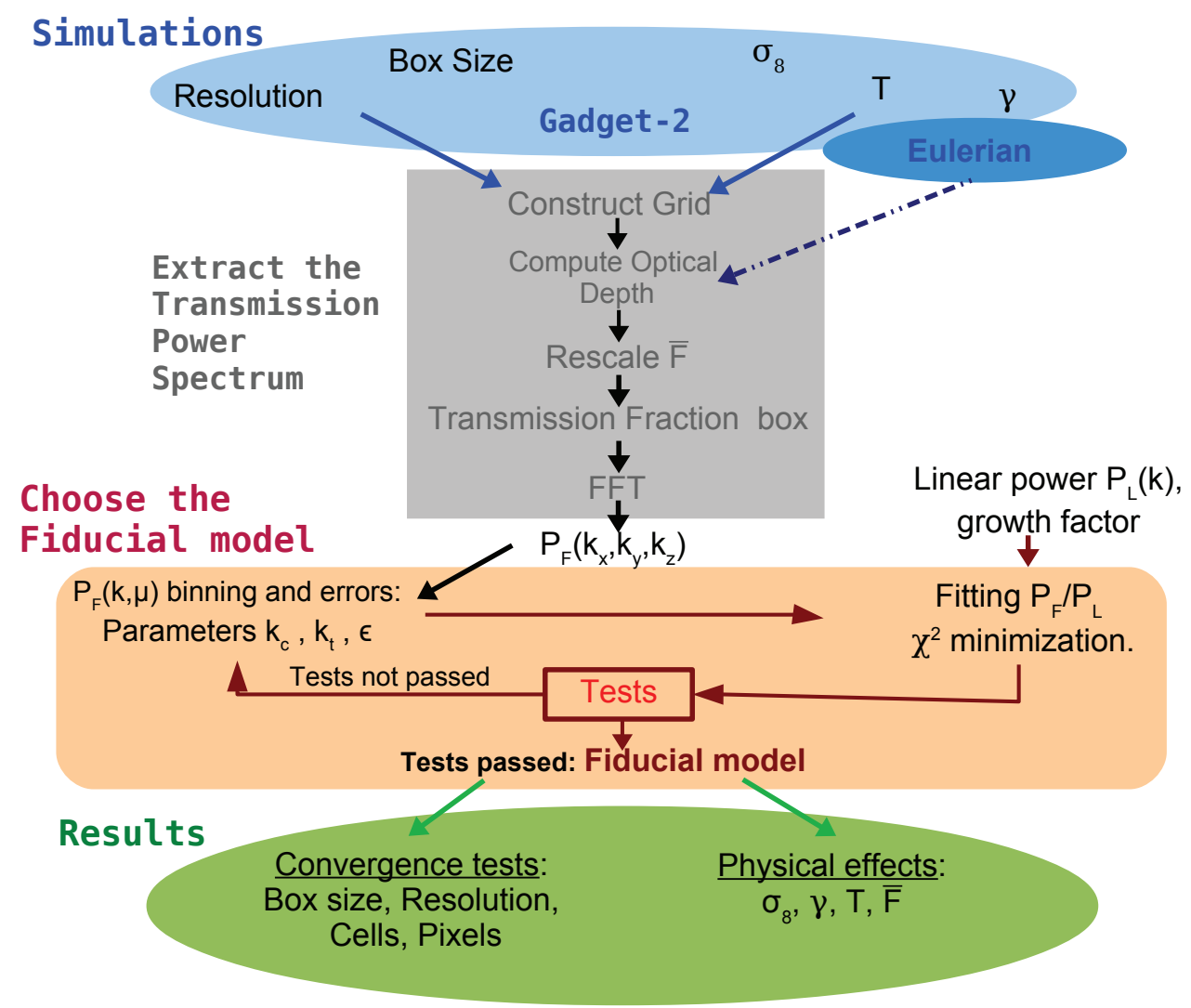

Figure 1. Flow chart of the analysis method of the power spectrum from simulations followed in this work. A set of GADGET-II simulations are used with different resolution and box sizes, and different physical properties: mean transmission $\bar{F}$, power spectrum amplitude $\sigma_{8}$, mean temperature $T_{0}$, and temperature-density relation slope $\gamma$. The output of the simulations at various redshifts is represented in a real space grid (the Eulerian simulation output directly provides this grid), and then the Ly $\alpha$ optical depth is computed in redshift space. These optical depths are rescaled to keep the mean transmission fraction fixed, and then the Fast Fourier Transform is performed and the square moduli provide estimates for the power spectrum $P_{F}\left(k_{x}, k_{y}, k_{z}\right)$. The power spectrum is then averaged in bins of $(k, \mu)$, errors are estimated, and fits to our proposed equation (3.6) are done. At first, tests of convergence with parameters related to the binning of Fourier modes and the errors are carried out. After these tests are performed, a fiducial model is chosen and further convergence tests are done for the simulation box size, resolution, and grid cells. Finally, the dependence of the results on physical effects is examined.

We will generally present results at the redshifts $z=2.2,2.4,2.6,2.8$, and 3 , where equation 3.1 implies rescaling the Ly $\alpha$ spectra to mean transmission values of $\bar{F}=0.8517$, $0.8185,0.7813,0.7404$, and 0.6960 , respectively. We shall use these values except for a few cases discussed in section 6.3 , where we examine the variation of $P_{F}$ under changes in $\bar{F}$.

A Fast Fourier Transform is applied to the entire box of Ly $\alpha$ spectra, for each of the three cases taking each axis as the LOS. Usually, the grid is cubic with $N_{c}^{3}$ cells, except in the P1024 model in Table 3.1 where the number of pixels is $2 N_{c}$. For the latter case, we first average the value of $F=\exp (-\tau)$ in every two pixels to obtain a cubic grid, and then compute the Fourier transform. The routine FFT from the scipy package in Python is used 
for this computation ${ }^{2}$. This results in $N_{c}^{3} / 2$ independent Fourier modes for each of the three axes chosen as the LOS, each one with a modulus and a phase. The moduli are used to obtain the estimate of the Ly $\alpha$ power spectrum $P_{F}(k, \mu ; z)$.

\subsection{Power spectrum estimation, Fourier space binning, and errorbars}

We discuss in this subsection the procedure for evaluating the power spectrum in Fourier space bins, using the Fourier modes of the Ly $\alpha$ transmission field of the simulations. The wavenumbers that are available in a simulation of box size $L$ go from the minimum value, $k_{1} \equiv$ $2 \pi / L$, to the maximum value equal to the Nyquist frequency, $k_{1} N_{c} / 2$. Several parameters are involved in our choice of binning in Fourier space and the computation of error bars of $P_{F}$ used to obtain fits, which we enumerate here:

1. The cutoff scale $k_{c}$. This is the maximum scale at which we consider that $P_{F}$ can be reliably predicted from a simulation and measured from the observations. We use only modes with $k<k_{c}$ to fit the results of $P_{F}$, where $k_{c}$ is less than the Nyquist value. For our fiducial simulation, the comoving cell size is $L / N_{c}=0.12 h^{-1} \mathrm{Mpc}$, corresponding to a velocity width $\sim 12 \mathrm{~km} \mathrm{~s}^{-1}$ and a Nyquist value $\pi N_{c}(1+z) /(H L) \sim 0.25 \mathrm{~s} \mathrm{~km}^{-1}$. Most of the modes in a simulation have wavenumbers near the Nyquist value, which are affected by the absorption tails of high column density systems arising from highly non-linear collapsed structures that may not be correctly modelled in the simulations. Moreover, in practice the observed power at this large $k$ is highly sensitive to the presence of narrow metal lines, which are not included in the simulations. Following M03, we consider that any comparison of theoretical and observed power spectra is not reliable for wavenumbers above $k(1+z) / H \sim 0.1 \mathrm{~s} / \mathrm{km}$, which is approximately a fixed comoving scale in our examined redshift range We therefore choose, for our fiducial simulation, $k_{c}=100 k_{1}=10.47 \mathrm{~h} / \mathrm{Mpc}$, and for all simulations we keep the physical value $k_{c}=10.47 \mathrm{~h} / \mathrm{Mpc}$ fixed. All the modes with $k>k_{c}$ are discarded. This leaves, for the fiducial model, $4 \times 10^{6}$ independent Fourier modes with $k<k_{c}$ to be used in our analysis.

2. The transition scale $k_{t}$. For effectively computing a $\chi^{2}$ function to fit the estimated power spectrum from a simulation to an analytic model, having more than $10^{6}$ values from independent Fourier modes is still a very large number, and the vast majority of these Fourier modes are at high $k$. To reduce this number, we define bins in the $(k, \mu)$ variables to average the estimated power spectrum within each bin when $k$ is larger than a transition scale $k_{t}$. For $k<k_{t}$, different modes obtained from the simulation are averaged only when they have exactly the same values of $(k, \mu)$, and compared to the power spectrum values from an analytic model at exactly the same $(k, \mu)$ to compute the $\chi^{2}$ function.

We choose $k_{t}=1 \mathrm{~h} / \mathrm{Mpc}$ for the fiducial simulation with box size $L=60 h^{-1} \mathrm{Mpc}$, which is close to the geometric average of $k_{c}$ and $k_{1}$. This results in roughly the same number of bins at $k>k_{t}$, as different values of $(k, \mu)$ at $k<k_{t}$, which optimizes the efficiency and accuracy of the calculation. For different box sizes, we keep fixed the value of $k_{t} L=60$, so that the number of Fourier modes evaluated without binning remains roughly constant. For example, for our largest simulation with $L=120 h^{-1} \mathrm{Mpc}$, we use $k_{t}=0.5 h / \mathrm{Mpc}$.

\footnotetext{
${ }^{2}$ http://docs.scipy.org/doc/numpy/reference/routines.fft.html
} 
3. Number of bins in $(k, \mu)$. The range of $k$ from $k_{t}$ to $k_{c}$ is divided into 16 bins that are equally spaced in $\log k$, and $\mu$ is divided also into 16 linearly spaced, equal bins from 0 to 1 . This gives a total of 256 bins for $k>k_{t}$ for which the power spectrum is estimated, for all simulations. Simulations with larger box size, with a smaller value of $k_{t}$, have therefore a larger bin size in $\log k$. The number of different values of $(k, \mu)$ that are obtained at $k<k_{t}$ turns out to be 296 for our choice of $k_{t} L=60$, therefore a total of 552 fitting points are used for all simulations when fitting $P_{F}(k, \mu)$ to an analytic model.

4. Errors $\sigma_{P}(k, \mu)$. Evaluating the $\chi^{2}$ function requires assigning an error to each Fourier mode estimated from a simulation. We assume that the Fourier modes are independent, and therefore that our covariance matrix is diagonal. We keep a count of the number of Fourier modes, $n_{F}(k, \mu)$, contributing to each of the 552 values of $(k, \mu)$ (including all three projections we use of the simulation box in redshift space, so $n_{F}$ is always a multiple of 3). We then compute the error of each power spectrum evaluation according to:

$$
\sigma_{P}(k, \mu)=P_{F}(k, \mu)\left[1 / \sqrt{n_{F}(k, \mu)}+\epsilon\right],
$$

where $P_{F}$ is the estimated value of the transmission power spectrum. For $\epsilon=0$, this is the expected error owing to the Poisson variance associated with the number of independent modes available in the simulation. The constant $\epsilon$ is included to avoid an excessive weight to the overall fit from the modes with the highest values of $k$, following the procedure of M03. The number of modes below a certain value of $k$ grows as $k^{3}$, so if a reasonable value of $\epsilon$ is not included, any analytic fit will need to be very highly accurate for all the high-k modes before the low-k modes are of any importance in determining the minimum of the $\chi^{2}$ function. Unfortunately, there is no clear objective way to decide the value of $\epsilon$ that one should choose to obtain a fit that is adequately weighting the results of a simulation over the broad range of $k$ that is being probed, and the results of the fits depend on $\epsilon$. In our work we have chosen a constant value of $\epsilon=0.05$ for all the simulations and analyses. The optimal value of $\epsilon$ is discussed in Appendix A.

It is worth going through some examples of the number of independent modes available for the power spectrum estimate for the smallest wavenumbers, starting at $k_{1}=(2 \pi) / L$. For the direction parallel to the LOS (with $k_{x}=k_{y}=0$ and $k_{z}=k_{1}$, choosing the z-axis as the LOS), with $\mu=1$, only one independent Fourier mode is obtained (the mode with $k_{z}=-k_{1}$ is not independent because of the condition that the Ly $\alpha$ transmission field is a real function). Two independent modes perpendicular to the LOS, with $\mu=0$, are available, for $k_{x}=k_{1}$, $k_{y}=0$, and $k_{x}=0, k_{y}=k_{1}$. The next smallest modes have $k=\sqrt{2} k_{1}$, with two independent modes for $\mu=0\left(k_{x}=k_{1}, k_{y}=k_{1}\right.$, and $\left.k_{x}=k_{1}, k_{y}=-k_{1}\right)$, and four independent modes for $\mu=1 / \sqrt{2}$ (with either $k_{x}$ or $k_{y}$ being equal to $+/-k_{z}$ ). In general, eight independent modes are available for any values of $k_{x}, k_{y}$ and $k_{z}$ when they are all different from zero and $k_{x} \neq k_{y}$ (owing to the symmetry under two independent sign changes and under the exchange of $k_{x}$ for $k_{y}$ ), which are used to estimate the Ly $\alpha$ power for the values $k=\left(k_{x}^{2}+k_{y}^{2}+k_{z}^{2}\right)^{1 / 2}$, $\mu=k_{z} / k$. In this case, the estimate of the power at this $(k, \mu)$ will come from $n_{F}=24$ values, because eight independent modes are obtained for each of the three axes chosen as the LOS. For some modes, this number is further increased whenever several combinations of $\left(k_{x}, k_{y}, k_{z}\right)$ yield the same values of $(k, \mu)$ (e.g., for $k_{x}=3, k_{y}=4$ and $k_{x}=5, k_{y}=0$ ). There are 296 different values of $(k, \mu)$ that are obtained from a cubic box with $k L<60$. 
For the bins at $k>k_{t}$, the average values of $(k, \mu)$ of the contributing Fourier modes in each bin are stored, in addition to the mean power spectrum value. These average values are usually very close to the central values of the bin (because the number of modes included in each bin is large), but they are not exactly equal. The model of the power spectrum to be fitted is then evaluated at these average values instead of the bin center.

\subsection{Parameterized fitting function for the $\mathrm{Ly} \alpha$ power spectrum}

The Ly $\alpha$ transmission power spectrum obtained from the simulations will be fitted to the following analytic model:

$$
P_{F}(k, \mu)=b_{F \delta}^{2}\left(1+\beta \mu^{2}\right)^{2} P_{L}(k) D(k, \mu) .
$$

The first terms on the right hand side are derived from the linear perturbation theory of [29], as explained in $\S 2$, where $P_{L}(k)$ is the mass density fluctuation linear power spectrum, $b_{F \delta}$ is the density bias factor of the Ly $\alpha$ transmission, and $\beta$ the redshift distortion parameter. The function $D(k, \mu)$ is the deviation from linear theory due to non-linear evolution, so we expect $D$ to approach unity in the limit of small $k$.

We shall use two different fitting models for $D(k, \mu)$ in this paper, although we have tested many others before deciding on a formula that provides good fits. First, the expression used by M03, which we designate $D_{0}$, with a total of 8 free parameters,

$$
D_{0}(k, \mu)=\exp \left[\left(\frac{k}{k_{n l}}\right)^{a_{n l}}-\left(\frac{k}{k_{p}}\right)^{a_{p}}-\left(\frac{k \cdot \mu}{k_{v 0}\left(1+k / k_{v 1}\right)^{a_{v 1}}}\right)^{a_{v 0}}\right] .
$$

Second, the expression we shall use in most of our fits, $D_{1}$, is a new one that has only 6 free parameters, and actually only 5 are used in most of our fits. We make the ansatz that the non-linear correction should behave as $D-1 \propto k^{3} P_{L}(k)$ in the limit of small $k$, because perturbation theory predicts that the second order terms of $P_{F}$ should depend on integrals of products of four linear perturbations. First, we define the Fourier amplitude of linear density fluctuations as

$$
\Delta^{2}(k)=\frac{1}{2 \pi^{2}} k^{3} P_{L}(k) .
$$

The fitting formula $D_{1}$ that we use is

$$
D_{1}(k, \mu)=\exp \left\{\left[q_{1} \Delta^{2}(k)+q_{2} \Delta^{4}(k)\right]\left[1-\left(\frac{k}{k_{v}}\right)^{a_{v}} \mu^{b_{v}}\right]-\left(\frac{k}{k_{p}}\right)^{2}\right\} .
$$

These equations are to be understood as simple fitting formulae that have been found to provide useful fits to the numerically obtained power spectra from the simulations by experience. However, some physical motivation for the various terms can be provided as follows:

- Non-linear enhancement: The power spectrum is increased on scales near the onset of non-linearity, because non-linear collapse of structure tends to enhance the power relative to the linear prediction. For the $D_{1}$ formula, and ignoring for now the $\mu$ dependence, this term is $q_{1} \Delta^{2}(k)$, with the optional addition of the higher order term $q_{2} \Delta^{4}(k)$ that may be included to improve the fit. The constants $q_{1}$ and $q_{2}$ are dimensionless and control the importance of this non-linear enhancement. In the $D_{0}$ formula this term is $\left(k / k_{n l}\right)^{a_{n l}}$. The scale $k_{n l}$ at which non-linear effects start being important should roughly obey $\Delta^{2}\left(k_{n l}\right) \sim 1$, so the dimensionless constants $q_{1}$ and $q_{2}$ in the $D_{1}$ 
formula are expected to be of order unity. The local slope of the power spectrum near this non-linear scale at $z \simeq 2.5$ is $n_{\text {eff }} \simeq-2.3$, implying that $\Delta^{2} \sim k^{0.7}$ near this scale, which agrees with the typical value found for $a_{n l}$ in M03. In the limit of small $k$, the difference $D-1$ is proportional to $\Delta^{2}(k)$ in our new formula, so an extrapolation to $k$ values smaller than those probed by our simulations can give a reasonable prediction, while the formula $D_{0}$ of M03 is expected to overpredict $D-1$ for small $k$.

- Jeans smoothing: The gas pressure suppresses the power below the Jeans scale. Smallscale power is present in regions of high density, where the Jeans scale is reduced in the highly non-linear regime, but this should not greatly affect the Ly $\alpha$ forest which is mostly sensitive to moderately overdense structures. This power reduction is modelled by the isotropic term with the scale $k_{p}$ playing a role that is reminiscent of a Jeans scale, although this cannot be taken literally because our formulae are simply a fit to a highly non-linear numerical result. The $D_{0}$ formula has a free power-law $a_{p}$ for this term, but we have found that good fits are obtained by fixing $a_{p}=2$, and therefore we fix this in the $D_{1}$ formula.

- Line-of-sight broadening: Finally, non-linear peculiar velocities and thermal broadening cause a smoothing of the correlation along the LOS, and therefore a suppression of power that increases with $\mu$. This suppression was found in M03 to be well matched by a power-law dependence on $\mu$ inside the exponential, but several parameters had to be added to fit the $k$-dependence of this non-linear anisotropic term, the third one in the $D_{0}$ formula. We find that by multiplying this term by the same non-linear enhancement depending on $\Delta^{2}(k)$, a simple power-law dependence on $k$ also provides a good fit.

Therefore, our new formula $D_{1}$ has two advantages over $D_{0}$ : it has the correct behavior for $D_{1}-1$ in the limit of small $k$, and it reduces the number of non-linear parameters from eight to six, and in fact to five in most of the simulations analyzed in this paper where we will set $q_{2}=0$, still providing sufficiently good fits.

To calculate the fit of the values of $P_{F}(k, \mu)$ extracted from the simulations to equation (3.3), we use the Montecarlo Markov Chain method [hereafter, MCMC; see, e.g., 2, for a review on the Metropolis algorithm that we use] to minimize the $\chi^{2}$ function, which we compute as

$$
\chi^{2}=\sum \frac{\left(P_{s}-P_{m}\right)^{2}}{\left(\sigma_{P} P_{m} / P_{s}\right)^{2}}=\sum \frac{\left(P_{s}^{2} / P_{m}-P_{s}\right)^{2}}{\sigma_{P}^{2}},
$$

where $P_{s}$ is the power spectrum measured from the simulation, $P_{m}$ is the power spectrum of the model being fitted, and the sums are over all the bins in $(k, \mu)$. The errors $\sigma_{P}$ are computed using equation (3.2) with $P_{F}=P_{s}$, so that they do not depend on the model, and are the ones shown in our figures. However, the $\chi^{2}$ function is obtained with the errors computed from the fitted model, which are $\sigma_{P} P_{m} / P_{s}$.

\section{Results: the L120 Simulation}

This section presents the results of the non-linear Ly $\alpha$ transmission power spectrum for the L120 simulation, the largest SPH simulation we have analyzed, with $768^{3}$ particles (see Table 3.1). We start using the fitting formula $D_{1}$ in equation (3.6), which will be used in all our results for other simulations. Figure 2 shows the estimated ratio $P_{F}(k, \mu) / P_{L}(k)$ at $z=2.2$ as colored points with error bars, and the resulting fits as curves, in the left panel. The 
non-linear term $D(k, \mu)$ is shown in the right panel. The colored curves are the fit to the 6 free parameters in $D_{1}(k, \mu)$ plus the two linear bias parameters, and the black curves show the fit that is obtained when the $q_{2}$ parameter, multiplying the second-order term in $\Delta^{2}(k)$, is set to zero.

The structure of these two panels will be the same for the various models analyzed in the rest of this paper. A set of four curves and points are shown for each fit, corresponding to the four intervals of $\mu$ indicated in the figure. An additional two curves show in this case the fit model at $\mu=0$ and $\mu=1$. The points are obtained by averaging the modes within the bins in $k$ that are plotted and these four bins in $\mu$. Even though the fit is performed with 16 bins in $\mu$ at $k>k_{t}$, as described in $\S 3.3$, the results are then further averaged into 4 bins for the purpose of display only. The fit also uses, as described above, 16 bins in $\log k$ within $k_{t}<k<k_{c}$, which are directly plotted, and individual mode values for $k<k_{t}$. These individual mode values are also averaged, for display purposes, into 16 bins in $\log k$ between $k_{1}=2 \pi / L$ and $k_{t}$, allowing for easy visualization of the results in plots that are similar to those in M03. Note that, for small $k$, some of the bins in $\log k$ and $\mu$ do not include any of the actual values of $(k, \mu)$ from the simulation modes, and in this case they are absent from the plot.

The way in which the values and errors of the power spectrum estimates in the original bins used for the fit are averaged into the bins used to make the figures is as follows: each original bin is assigned a weight $w(k, \mu)=1 / \sigma_{P}^{2}(k, \mu)$, using the errors in equation (3.2). The values of the power spectrum and the mean coordinates of the coarse bins for the plot are obtained by averaging $P_{F} / P_{L}, \log k$ and $\mu$ with these weights, and the new error in the coarse bin is set to the inverse square root of the sum of the weights $w(k, \mu)$, assuming Gaussian independent errors. The error bars therefore indicate the effective weight that each plotted point, as the average of several points used in the actual fit, is given to obtain this fit.

The curves are the result of the model fit, when the model is computed on the same values of $(k, \mu)$ of all the bins used for the fit, and then averaged in the same way as the simulation points for display purposes. This averaging is the reason for the discontinuities in these curves in the left panel, which are particularly apparent at low $k$. The four curves for the four values of $\mu$ start from the left side at different values of $k$, depending on the smallest $k$ value for which there exists a mode having $\mu$ within each bin. The smallest wavenumber, with $k=k_{1}$, exists only for $\mu=0$ and $\mu=1$. The model predictions are shown for the same values of $(k, \mu)$ as the points, and are plotted as a continuous line only to guide the eye. Finally, the cyan and brown dash-dot lines (black for the $q_{2}=0$ case) are the model predictions for $\mu=0$ and $\mu=1$ as a function of $k$ (this time, not averaging over any bins), shown to indicate the difference with the results in the averaged bins of smallest and largest $\mu$.

When the points indicating simulation results and the curves showing the model in the left panel are divided by the expression $\left[b_{F \delta}\left(1+\beta \mu^{2}\right)\right]^{2}$ in each of the computational bins, using the bias factors obtained in each fit, the non-linear term $D(k, \mu)$ is obtained, plotted in the right panel. The colored points are now valid only for the free $q_{2}$ fit; points for the $q_{2}=0$ fit are omitted to avoid excessive cluttering. As before, the points and model predictions are averages of the bins used for computing the fit over the coarser bins used to make the plot. The averaging of the values and errors is done using the same weights as for the ratio $P_{F} / P_{L}$.

Figure 2 shows that a very good fit is obtained by varying only the 6 parameters in equation (3.6), plus the two linear bias factors (colored curves). A value of $\chi^{2}=296.7$ is obtained, for $552-8=544$ degrees of freedom at redshift 2.2. When fixing $q_{2}=0$ at $z=2.2$, 

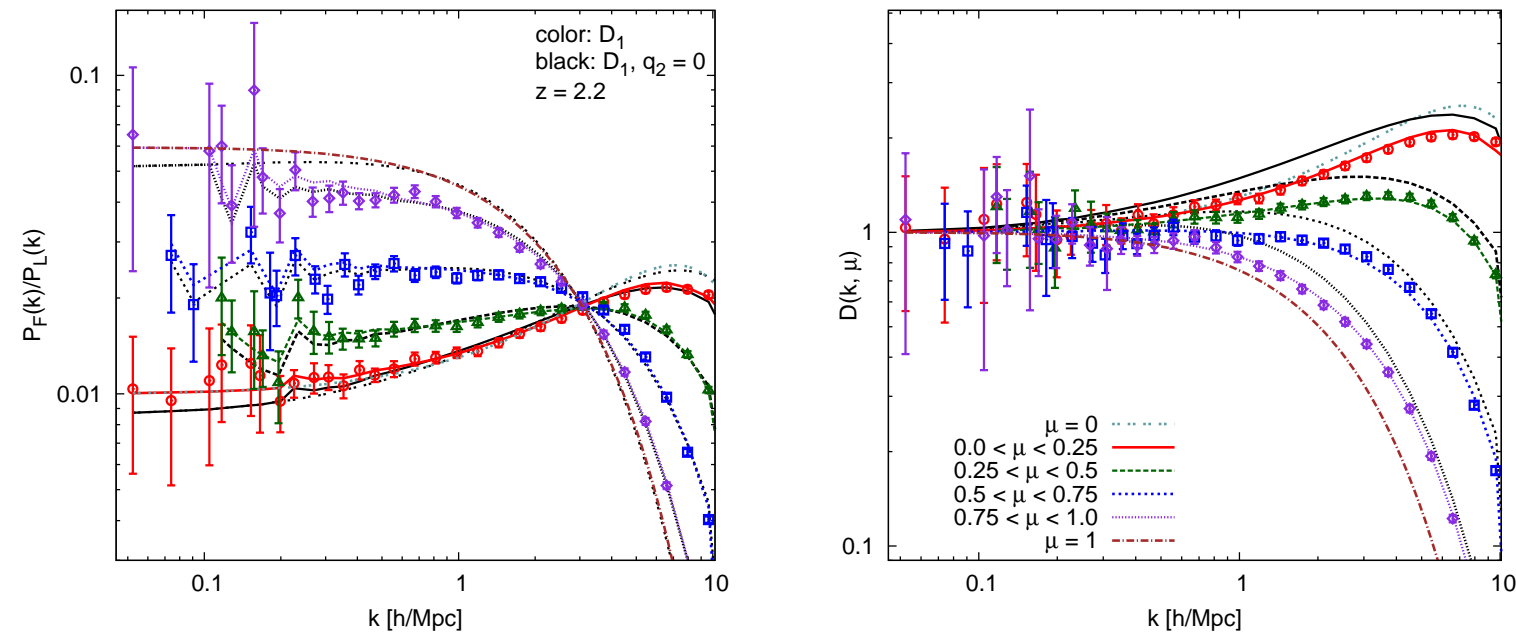

Figure 2. Power spectrum for the $120 \mathrm{Mpc} / h$ box at $z=2.2$, averaged over the indicated 4 bins in $\mu$, and with bins in $\log k$ as described in the text. Points with error bars in the left panel are the results from the simulation, and colored lines are the fit to equation (3.6) with 6 free parameters (in addition to the two bias factors). Black curves are the fit to 5 free non-linear parameters, when setting $q_{2}=0$ in $D_{1}$. The left panel shows the ratio of the transmission power spectrum to the linear one, and the right panel shows the non-linear term $D(k, \mu)$. Points in the right panel are shown only for the fit with $q_{2}$ as free parameter, to avoid cluttering. Brown dashed curves are the fitted model computed at $\mu=0$ and $\mu=1$, shown to indicate the difference with the averaged bins $0<\mu<0.25$ and $0.75<\mu<1$; black dashed curves (shown only in the left panel) are the same for the fit with $q_{2}=0$.
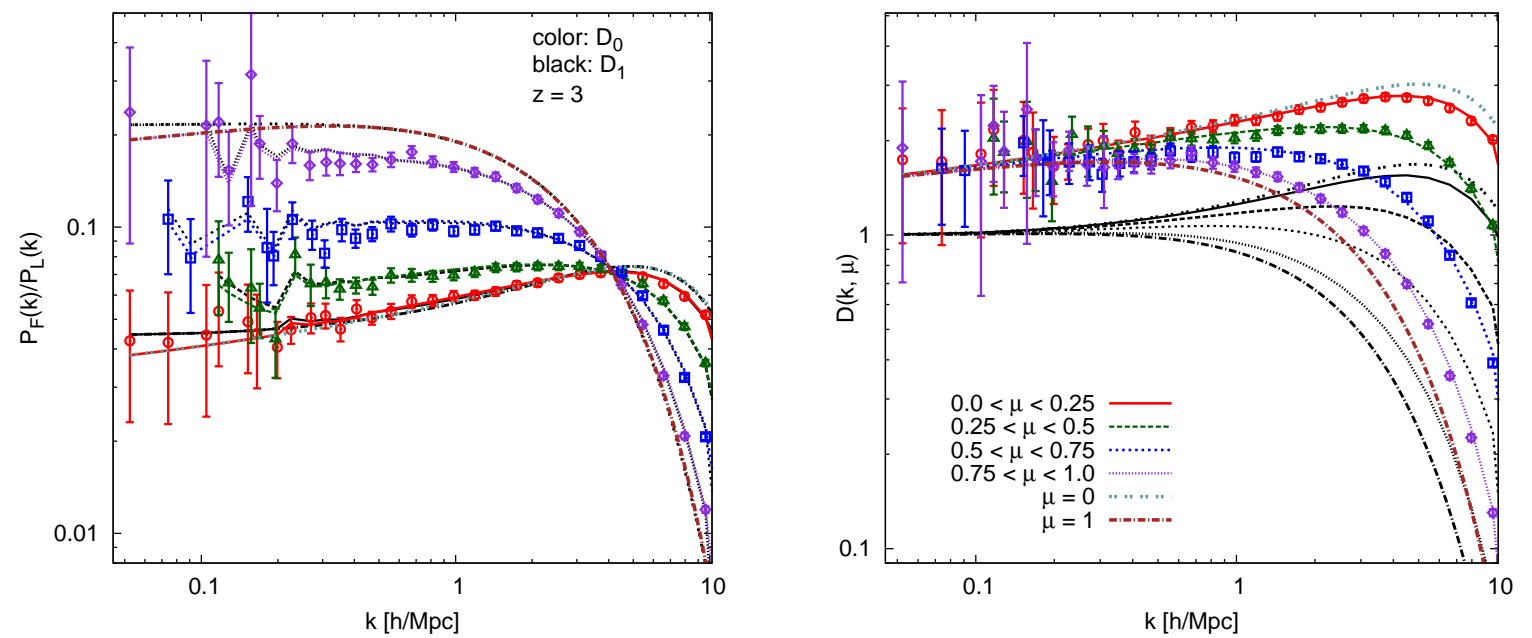

Figure 3. Same as figure 2, but comparing the fit using the $D_{0}$ formula (colored curves and points) with the one using $D_{1}$ with free $q_{2}$ (black curves; black points omitted in right panel), and at $z=3$.

the value of $\chi^{2}$ increases to 349.8. This value of $\chi^{2}$ does not reflect a real "goodness of fit" because we have added the parameter $\epsilon$ in equation (3.2) to reduce the weight of the modes at high $k$, and because the initial conditions were generated without including the Rayleigh distribution in the amplitude of each Fourier mode, so the scatter of the values of $P_{F} / P_{L}$ 
arises only from non-linear coupling. The value of $\chi^{2}$ is therefore substantially less than the number of degrees of freedom. Nevertheless, its variation with the model fit can still indicate if the improvement of a fit is significant. The fit with $q_{2}=0$ is already quite good, but it improves substantially by including $q_{2}$ as free parameter. In particular, the low- $k$ points are better matched for free $q_{2}$, increasing the model power at small $k$ by $\sim 15 \%$, implying an increase of $\sim 7 \%$ in the density bias factor. The difference between the two fits for $D(k, \mu)$ in the right panel goes up to nearly $20 \%$ and extends to higher $k$, because of degeneracy of the non-linear parameters with the linear bias factors.

Results at the higher redshift $z=3$ are shown in Figure 3, this time comparing the fitting formula $D_{1}$ with all 6 free parameters (black curves), with the result for $D_{0}$ with 8 non-linear parameters (colored curves). As before, black points are omitted in the right panel. The fits are fairly close to each other for $P_{F} / P_{L}$, with values of $\chi^{2}=252.0$ for $D_{1}$, and $\chi^{2}=262.3$ for $D_{0}$, again with a difference at low $k$ where $D_{1}$ better matches the simulation points. In this case, the fit with $D_{1}$ fixing $q_{2}=0$ is much closer to being optimal than at $z=2.2$, with $\chi^{2}=254.6$. The fit with the $D_{1}$ formula is clearly better than with $D_{0}$, with two fewer parameters; we have found this to be true also at $z=2.2$. Moreover, we see in the right panel that the formula $D_{0}$ converges very slowly toward unity at small $k$. The reason is that the value of the $a_{n l}$ parameter in equation (3.4) obtained for this fit at $z=3$ is small, $a_{n l} \simeq 0.2$, and there is a strong degeneracy with the value of the linear bias factors. This formula therefore easily leads to unphysical parameter values when the linear bias factors are not determined independently from the fit to the power spectrum of a simulation of limited box size. The values of the parameters are listed in the tables in Appendix B.

\subsection{Results for the linear bias factors}

In the limit of small $k$, the ratio $P_{F} / P_{L}$ approaches $b_{F \delta}^{2}\left(1+\beta \mu^{2}\right)^{2}$. This is indeed the behavior shown by our results in the left panel of Figures 2 and 3 . The size of the box limits the number of Fourier modes available in the simulation at low $k$ and hence the accuracy to which the linear bias factors can be measured from our fit. In addition, degeneracies between these bias factors and the non-linear parameters are present when fitting the simulation results for $P_{F} / P_{L}$.

The values of the bias factors and the redshift distortion parameter are shown in Figure 4 at the 5 redshift outputs that we will use in most of our models, and for the three cases we have considered: the $D_{1}$ formula with free $q_{2}$, fixing $q_{2}=0$ in $D_{1}$, and the $D_{0}$ formula. The two physical bias factors, $b_{\tau \delta}$ and $b_{\tau \eta}$, are obtained from the transmission bias factors $b_{F \delta}$ and $b_{F \eta}$ derived from our fits of $P_{F}(k, \mu) / P_{L}(k)$ through equation (2.4), and are shown in the center and right panels. Errorbars are derived from the Monte Carlo Markov Chain computed for the fits, and they generally overestimate the purely statistical errors for a fixed fitting function because our $\chi^{2}$ function is too low due to the $\epsilon$ parameter and the absence of Rayleigh-distributed amplitudes in the simulation. However, they are dependent on the fit model and they do not include the systematic errors that are investigated in $\S 5$.

The results of the bias factors for $D_{0}$ differ substantially from the ones derived with $D_{1}$, and they are less reliable because the function $D(k, \mu)$ converges too slowly towards unity at small $k$, as seen in Figure 3. This slow convergence is unphysical because non-linear effects are actually very small on the largest modes of the L120 simulation box. This problem for $D_{0}$ is worse at high redshift and, together with strong degeneracies with the non-linear parameters, is the cause of the strange redshift dependence of the bias factors. For this reason, we focus in the rest of this paper on results obtained with $D_{1}$. 

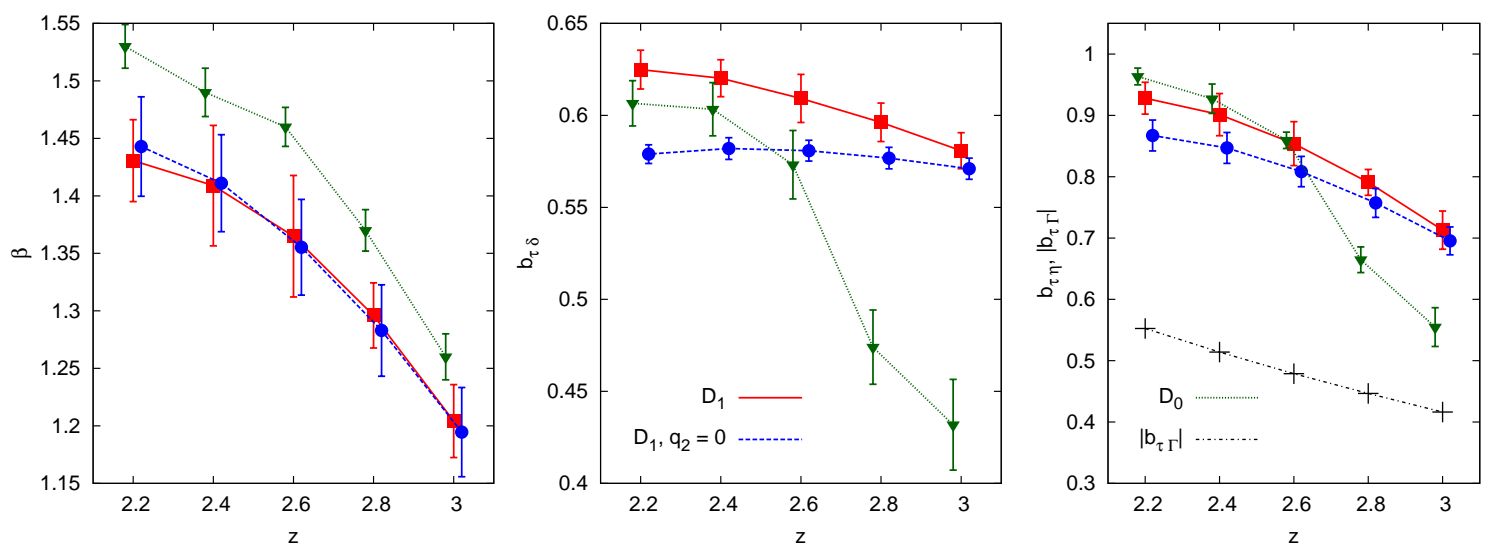

Figure 4. Results of the fitted values for the two bias factors $b_{\tau \delta}$ and $b_{\tau \eta}$, and the redshift distortion parameter $\beta$ as a function of redshift, for the 5 redshifts outputs of the L120 simulation. The error bars are $1 \sigma$, as returned from the MCMC fitting. To avoid superposition of errorbars, green points are shifted slightly left and blue points slightly right, but they are all at our 5 standard redshift outputs. Results are shown for the fitting formula $D_{1}$ with all 6 free non-linear parameters (red squares), for $D_{1}$ setting $q_{2}=0$ (blue circles), and for the formula $D_{0}$ with 8 free parameters (green triangles). The differences among the fitting models are caused by degeneracies with the non-linear parameters, which are more severe for $D_{0}$. In the right panel, the absolute value of the radiation bias parameter $b_{\tau \Gamma}$ is also shown as crosses.

The redshift distortion parameter $\beta$, shown in the left panel, is predicted to have a value near 1.4 at the most commonly observed redshift in BOSS, $z \simeq 2.3$, and to decline with redshift. There is little variation of the value of $\beta$ when fixing $q_{2}$ to zero. The value we predict is slightly smaller than that of $\mathrm{M} 03$, who found $\beta=1.58$ at $z=2.25$. Our result for $\beta$ for the $D_{0}$ formula is closer to that of M03, but this is not for the same reason since an independent method was used by M03 to determine the bias factors. The dependence of $\beta$ on the fitting method and physical parameters will be further discussed in $\S 6$.

The physical bias parameters have a relatively weak dependence on redshift. Previous results reported in terms of the transmission bias factors indicated a very rapid evolution with redshift, due mostly to the change in the mean transmission $\bar{F}$. The predicted value of $b_{\tau \delta} \simeq 0.6$, nearly constant with redshift, has the physical meaning that the Ly $\alpha$ effective optical depth fluctuates on large scales by $\sim 60 \%$ of $\delta$, the fluctuation in the mass density field. As mentioned above, the density bias factor drops by $\sim 8 \%$ at $z=2.2$ when fixing $q_{2}=0$, corresponding to the $\sim 15 \%$ variation of power at low $k$ seen in Figure 2 . This is an indication of the uncertainty due to the degeneracy with non-linear parameters and the use of different fitting formulae.

The bias factor of the peculiar velocity gradient is computed from equation (2.6). For the logarithmic derivative of the growth factor, we use the values for the cosmological model of our fiducial simulation: $f(\Omega)=(0.9875,0.9895,0.9911,0.9924,0.9935)$ at $z=(2.2,2.4$, $2.6,2.8,3)$. The value of $b_{\tau \eta}$ is below unity and decreases with redshift. This means that the Ly $\alpha$ forest behaves differently from a large-scale structure survey of objects with a selection function that is independent of the peculiar velocity gradient along the LOS, $\eta$ : the effective optical depth fluctuates only by $\sim 70 \%$ of the fluctuation in $\eta$ at $z=3$. We shall return in $\S 7$ to the physical reason why $b_{\tau \eta}$ is less than unity and decreases with redshift, which is a general characteristic in the results of all our simulations. 
Finally, we compute also the radiation bias factor $b_{\tau \Gamma}$, defined at the end of $\S 2$, using the probability distribution of $F$ in the simulation L120 at each redshift output, and equation (2.8). The results are shown as crosses in the right panel of figure 4. The values are a factor of $\sim 2$ smaller than $b_{\tau \eta}$. This shows that the approximation proposed by [51], in which $b_{\tau \eta}=b_{\tau \Gamma}$, fails in an important way. Following on our discussion at the end of $\S 2$, we believe the reason is that non-linear evolution of the small scale fluctuations in the Ly $\alpha$ forest substantially modifies the value of $b_{\tau \eta}$.

As far as the linear power spectrum is concerned, the main goal of numerical simulations of the Ly $\alpha$ forest should be to accurately predict the value of $\beta(z)$ and $b_{\tau \eta}(z)$, and to examine the model dependence of these functions, to compare to observational determinations. Our results in this section already show the main difficulty involved in this goal: the numerical fits we obtain depend on the fitting formula that is used, and on the value of the $\epsilon$ parameter we have chosen in equation (3.2) to increase the importance of the low- $k$ modes. When setting $q_{2}=0$, the fit to the low- $k$ points is worse and both $b_{\tau \delta}$ and $b_{\tau \eta}$ go down. However, if $q_{2}$ is set free then the $q_{1}$ parameter determining the limit of the non-linear correction at low $k$ becomes highly degenerate. Determining accurate values of the bias parameters with a reliable non-linear correction can only be done with large numbers of simulations on large boxes, to have better statistics for the power at low- $k$. Since we have only one simulation with $L=120 h^{-1} \mathrm{Mpc}$ and a few on smaller boxes, the results in this paper on linear bias factors are of limited accuracy, as exemplified by the difference in our two different fits with $D_{1}$.
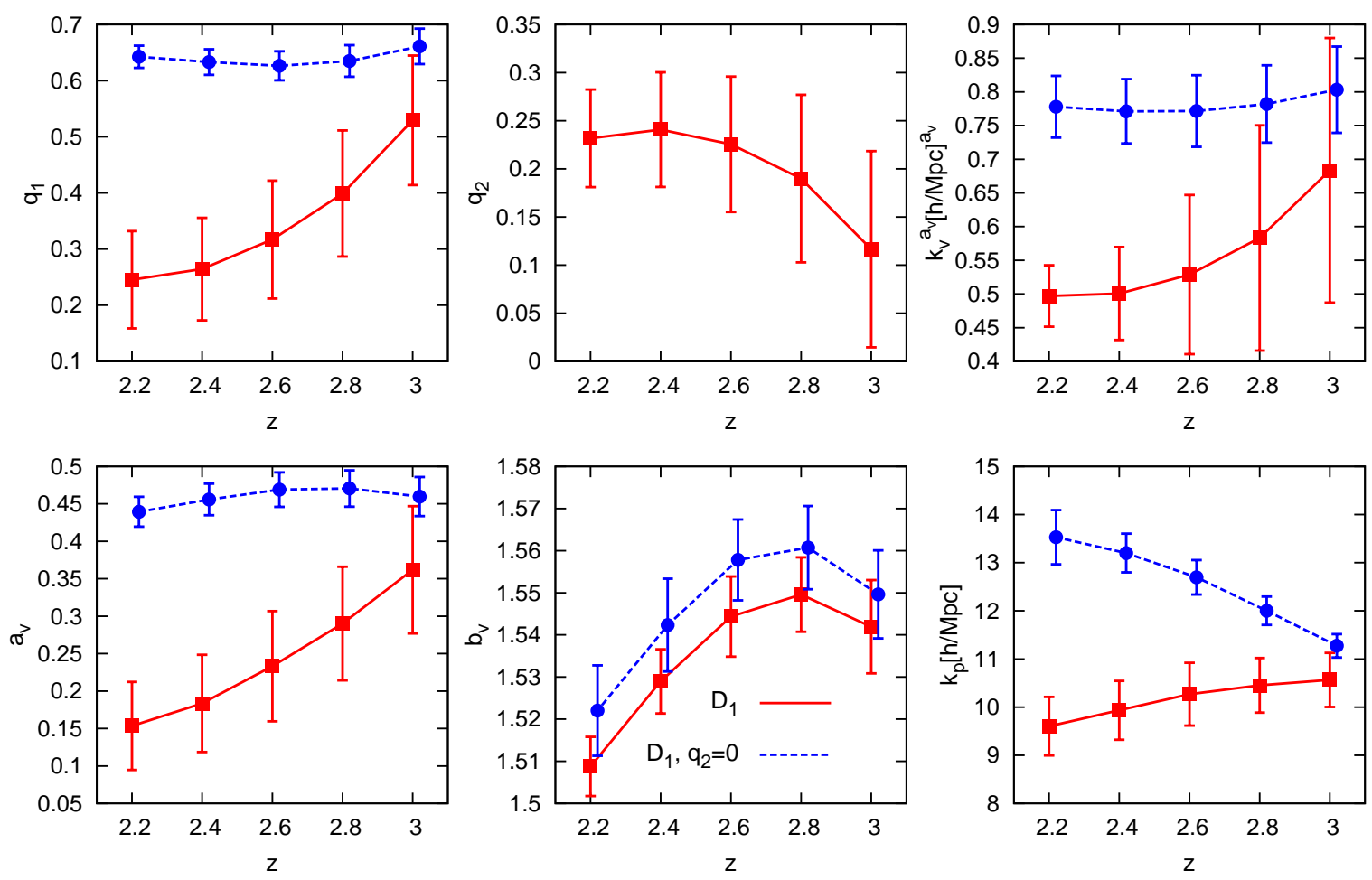

Figure 5. Results of the non-linear parameters of $D_{1}$ as a function of redshift, for the 5 redshifts outputs of the L120 simulation. The error bars represent the $1 \sigma$ contour as obtained from the MCMC fitting. Solid red lines are for all 6 parameters left free, and dashed blue ones are for fixing $q_{2}=0$. 


\subsection{The non-linear part of the power spectrum}

The values of the non-linear parameters are shown for the fitting model $D_{1}$ in Figure 5 , for all 6 parameters being free (red solid line), and for $q_{2}=0$ (blue dashed line). We plot $k_{v}^{a_{v}}$ instead of $k_{v}$ to reduce the amount of degeneracy among parameters. We comment here on the principal features of the results on these parameters and what this reflects on the shape of the non-linear function $D(k, \mu)$.

The scale $k_{p}$ is generally near $10 \mathrm{~h} / \mathrm{Mpc}$, or $k_{p} / H \sim 0.1 \mathrm{~s} / \mathrm{km}$. This is a characteristic value for the Jeans scale of the photoionized gas in the IGM, as discussed in §3.4. This parameter is used to fit the declining power with $k$ that occurs above this scale even for $\mu=0$, clearly seen in Figures 2 and 3. The value of $q_{1}$, controlling the amplitude of the non-linear power enhancement, is $q_{1} \simeq 0.6$ when no second-order term is included, but a substantial degeneracy occurs with the parameters $q_{2}, k_{v}, k_{p}$ and $a_{v}$ when $q_{2}$ is included in the fit, causing large changes of $q_{1}$ that vary with redshift.

Non-linear effects imply a change of the sign of the quadrupole of $P_{F}(k, \mu)$ as $k$ increases. At small $k$, the power is largest at $\mu=1$ due to the Kaiser effect, and at large $k$ the effect of velocity dispersion takes over and the power is largest at $\mu=0$. The quadrupole is zero at a point where curves of different $\mu$ cross each other. This point reflects a characteristic scale at which non-linearity changes the sign of the power spectrum anisotropy, and shifts to the left (larger scales) as the amplitude of the mass power spectrum increases from $z=3$ to $z=2.2$.
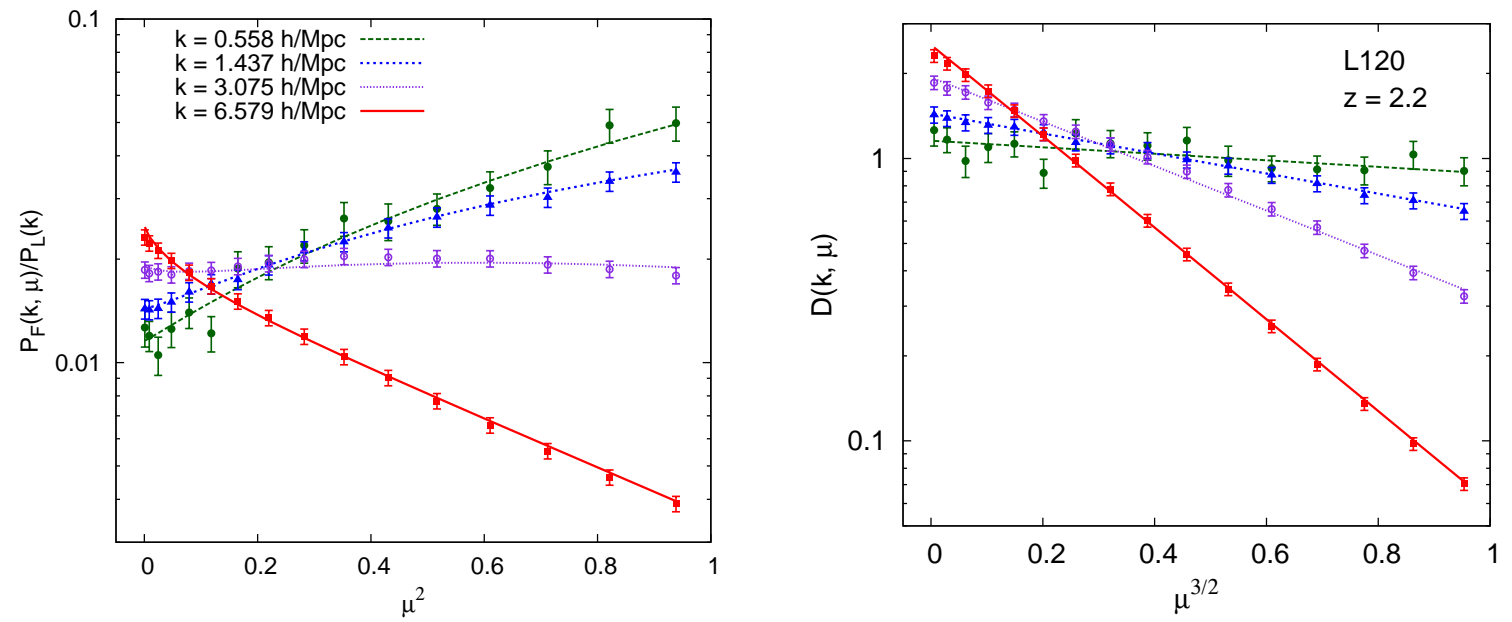

Figure 6. Left panel: $P_{F}(k, \mu) / P_{L}(k)$ as a function of $\mu^{2}$, for the bins centered at $k=$ $(0.56,1.44,3.08,6.58) h / \mathrm{Mpc}$ (or bins number $1,6,10$ and 14 above $k_{t}$ ). Right panel: same points for $D(k, \mu)$ as a function of $\mu^{1.5}$.

The dependence of the non-linear correction on $\mu$ is very well represented by the powerlaw $\mu^{b_{v}}$ inside the exponential in equations (3.4) and (3.6), where $b_{v}$ is close to 1.5 at all redshifts and is subject to little degeneracy with other parameters. To see in greater detail the dependence of the power on $\mu$, Figure 6 shows $P_{F} / P_{L}$ as a function of $\mu^{2}$ in the left panel, and $D(k, \mu)$ as a function of $\mu^{1.5}$ in the right panel, for a set of four selected bins in $\log k$, corresponding to bins number 3, 7, 11 and 16 in Figure 2 starting from the right edge. The four values of $k$ of these bins are indicated in the figure, in units of $h / \mathrm{Mpc}$. This figure shows the actual value of $P_{F}$ that we use in our fits for the 16 bins in $\mu$ in our analysis, instead of the averages over coarse bins as in previous figures. The results are shown at $z=2.2$ and for 
the $D_{1}$ fit including $q_{2}$ as free parameter. We see in the left panel that at the scale where the quadrupole changes sign $(k=3.04 h / \mathrm{Mpc})$, the power is indeed almost independent of $\mu$. The right panel shows that the $\mu$-dependence of the non-linear correction to the power is surprisingly well fit by a simple power-law at all values of $k$.

It will be interesting to express our non-linear parameters in terms of the characteristic scale where the anisotropy changes sign. We define the wavenumber $k_{n a}$ (the subindex is for the scale of non-linear anisotropy) as the one that obeys

$$
P_{F}\left(k_{n a}, \mu=0\right)=P_{F}\left(k_{n a}, \mu=1\right) .
$$

The $\mu$ dependence of the transmission power spectrum for our fitting formula $D_{1}$ is

$$
P_{F}(k, \mu) \propto\left(1+\beta \mu^{2}\right)^{2} \exp \left[-\beta_{a}(k) \mu^{b_{v}}\right],
$$

where, if we use for simplicity the formula $D_{1}$ with $q_{2}=0$, we have

$$
\beta_{a}(k)=q_{1} \Delta^{2}(k)\left(\frac{k}{k_{v}}\right)^{a_{v}} .
$$

The value of $k_{n a}$ can be computed from our non-linear parameters from equation

$$
\beta_{a}\left(k_{n a}\right)=2 \log (1+\beta) .
$$

As it turns out, the function of $\mu$ in equation (4.2) happens to be nearly constant in the range $0<\mu<1$ when $b_{v} \simeq 1.55$ and $\beta \simeq 1.4$, if $\beta_{a}$ obeys equation (4.4). As we shall see, these values of $b_{v}$ and $\beta$ do not change much for all the other models we examine in this paper. This is the reason that all the curves for $P_{F} / P_{L}$ with different $\mu$ cross each other nearly at the same wavenumber $k_{n a}$ for all our models.

We compute the value of $k_{n a}$ for all our models with $q_{2}=0$, by iteratively solving the equation

$$
k_{n a}=\left[\frac{(2 \pi)^{2} k_{v}^{a_{v}} \log (1+\beta)}{q_{1} P_{L}\left(k_{n a}\right)}\right]^{1 /\left(3+a_{v}\right)} .
$$

The parameter values of our fits are given in the tables of Appendix B. The parameter $k_{n a}$ is particularly useful because its correspondence with the crossing point of the curves means that it has very little degeneracy with all other parameters, and therefore its error is small.

We have not been able to think of an analytical explanation for the surprisingly well matched $\mu$-dependence of $\log D(k, \mu)$ to the power-law $\mu^{b_{v}}$, with $b_{v} \simeq 1.55$. We note that $b_{v}<2$ implies a singular second derivative of $P_{F}(k, \mu)$ at $\mu=0$.

Finally, we also note that once $q_{2}$ is introduced as a free parameter, the power-law index $a_{v}$ becomes very small, particularly at low redshift, and a large degeneracy is introduced among the parameters $k_{v}, a_{v}, q_{1}$ and $q_{2}$. The improvement in the fit obtained by including $q_{2}$ is modest, and this improvement is further reduced for simulations in smaller boxes, where less information is available on the $k$-dependence (and the parameter degeneracy increases). We therefore will fix $q_{2}=0$ for our fits to the results of most of our simulations.

\section{Convergence Tests: Resolution, Box Size and Numerical Method}

This section addresses the degree to which our fit results from the various simulations we analyze have converged when box size, resolution, grid size and numerical method are varied. 
Variations of the power spectrum with physical parameters of the Ly $\alpha$ forest will be discussed in $\S 6$.

We remark here one important difference between our work and that of M03. We do not use any splicing technique (see also [7]) to combine results from simulations of different resolution or box size, a method introduced by M03 to attempt to better reach a convergent solution. In this work we simply fit both the linear bias factors and the non-linear parameters of the analytic formulae of equations (3.4) and (3.6) to the power spectrum of each simulation. Our results can probably be improved by using this splicing technique, in particular for the linear bias factors, but we did not have enough simulations in this work to do this for all the models we analyze, and we left this for future studies. The emphasis of our analysis is more focused on the variations of the bias parameters and the non-linear form of the Ly $\alpha$ power spectrum with respect to a reference model (our fiducial simulation), rather than aiming for a highly accurate convergence of the results, for which a larger number of simulations on large boxes are required.

Before we start, we also note that all our SPH simulations generally use the same random initial conditions for the phases of Fourier modes of fixed $k L$. Moreover, as described in $\S 3.1 .1$, the amplitudes of the modes are always equal to the variance predicted by the power spectrum, instead of being generated randomly with the Rayleigh distribution. This minimizes the random sampling variance due to the finite box size and allows for a more direct comparison of different models. In general, however, there is no guarantee that the average $P_{F}$ obtained by suppressing the Rayleigh distribution of amplitudes in the initial conditions is the same as the correct $P_{F}$ that can be derived only by including the Rayleigh distribution, and this will need to be tested in future studies. As we shall see in this section, the variations of the low- $k$ power spectrum from simulations of the same model but different random initial conditions are similar to the differences introduced by resolution and box size, and the overall accuracy to which we can measure the bias factors from our simulations is not better than $\sim 10 \%$.

\subsection{Resolution}

We start by checking the dependence of our results on the resolution. There are three different quantities in relation to the resolution of the simulation and the analysis that is done for computing the power spectrum that must be tested for convergence: the number of particles in the simulation, the number of cells in the grid to compute the hydrodynamic variables, and the number of pixels in the simulated Ly $\alpha$ spectra.

We compare first results for the power spectrum from the same simulation and spatial grid, but varying the pixel resolution of the computed Ly $\alpha$ spectra. Results for $P_{F} / P_{L}$ are shown in figure 7 , where black curves are for our fiducial model and colored curves and points are for the P1024 model. The fiducial model is the same as the one used in $\S 4$, but with the box size $L=60 h^{-1} \mathrm{Mpc}$, and the P1024 model is exactly the same but with the number of pixels in the Ly $\alpha$ spectra doubled to 1024 (Table 3.1). Black points are omitted to avoid cluttering, but they follow the black curves similarly to the colored ones. The P1024 model is computed by calculating the optical depth in 1024 pixels along the LOS, and then averaging $\exp (-\tau)$ for every two pixels to obtain a cubic grid of $512^{3}$ values of the transmission fraction, on which a Fast Fourier Transform is done in the same way as for all our other models. Both models are fitted with our $D_{1}$ formula with $q_{2}=0$.

The results are shown at $z=2.6$, and are very similar at other redshifts. The increase in the pixel resolution practically does not affect the transmission at the level that is discerned 


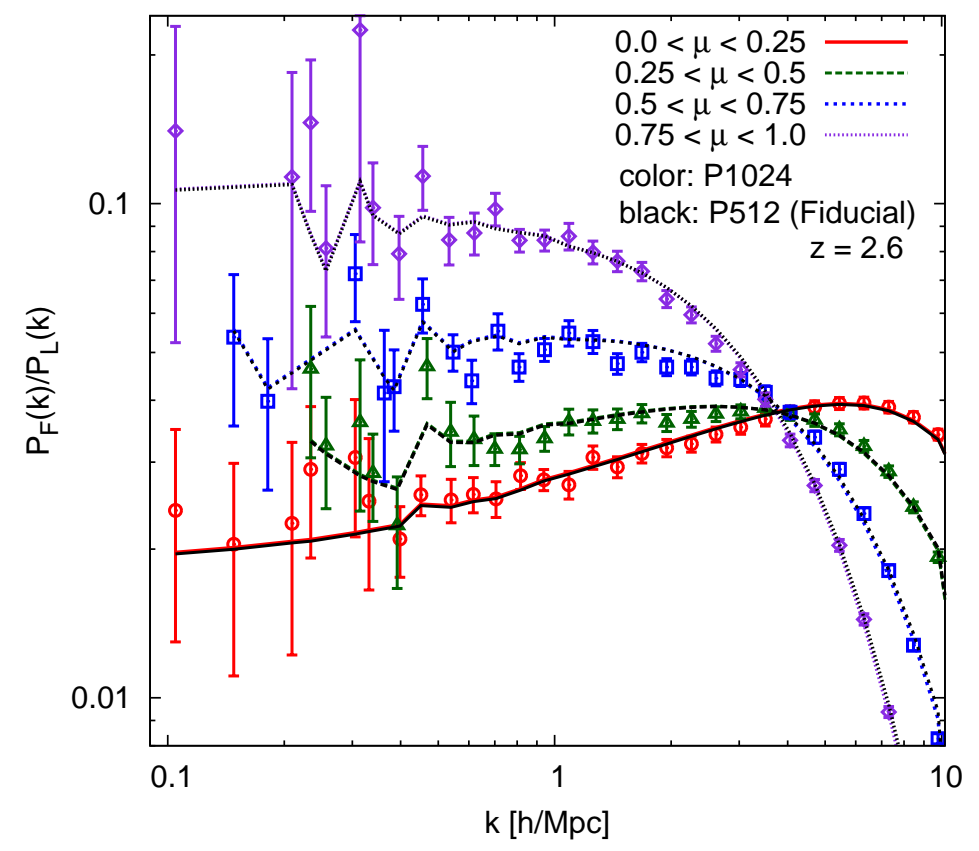

Figure 7. Comparison of the power spectrum result obtained for our fiducial simulation and model analysis (black curves; box size $L=60 h^{-1} \mathrm{Mpc}, 512^{3}$ particles and grid cells, and 512 spectral pixels), with the case of doubling the spectral pixels to 1024, at redshift $z=2.6$ (colored curves). Black points are omitted to avoid cluttering.

in figure 7 (the variation is less than $2 \%$ ). Clearly, the spectral pixel resolution is sufficient for our purpose, and any errors it is causing are much smaller than those due to the grid size and number of SPH particles in the simulation.

Next, we compare two analyses of a simulation using spatial grids of different resolution. For that purpose, we take our highest physical resolution simulation, R640 (equal to the fiducial one but with $640^{3}$ particles instead of $512^{3}$, although still analyzed on a $512^{3}$ cells grid; see Table 3.1), and compare it to R640C, the same simulation analyzed on a grid of only half the sampling, with $256^{3}$ cells. The results for $P_{F} / P_{L}$ are shown in figure 8 , at redshift $z=2.2$. Larger differences can be appreciated in this figure. First of all, a coarse grid increases the power on small scales, $k>5 h / \mathrm{Mpc}$, probably due to the noise introduced by a grid cell of $0.23 h^{-1} \mathrm{Mpc}$ in R640C. This also generates a roughly constant decrease of the power on large scales in R640C compared to R640. In fact, for $k<0.5 h^{-1} \mathrm{Mpc}$, the black points are systematically higher than the colored ones by $\sim 7 \%$ at all values of $\mu$. The change in the small-scale power alters non-linear couplings to produce an impact on the large-scale power that becomes constant on very large scales, therefore altering the bias factors. Results at other redshifts are qualitatively similar.

Figure 8 also exemplifies the uncertainty in our fits for recovering the low- $k$ power: the colored curves move above the black ones at high $\mu$ and low $k$, which is opposite to the simulation results for $P_{F} / P_{L}$ indicated by the points. This is again reflecting the uncertainty due to the fitting formula that is chosen. The fitted curves can better reflect the behavior of the simulation points when the $q_{2}$ parameter is left free, but the reliability of the derived bias factors is still subject to a similar error because the small box size does not allow one to obtain the bias factors more accurately. 


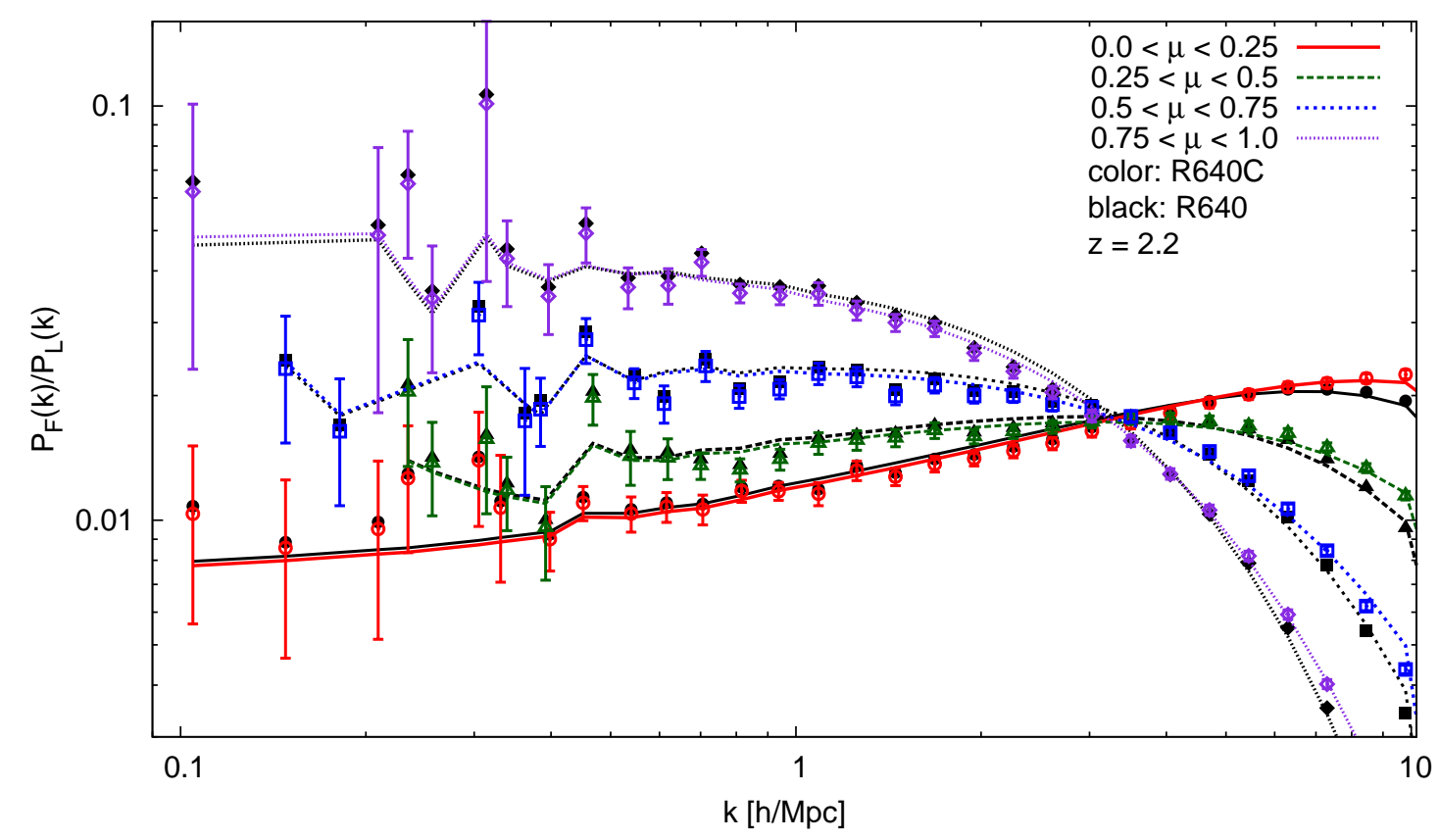

Figure 8. Comparison of $P_{F} / P_{L}$ at $z=2.2$, for the R640 simulation with $640^{3}$ particles analyzed with a grid of $512^{3}$ cells (black curves and points), with the same simulation analyzed with a coarser grid of $256^{3}$ cells (R640C, colored curves and points). Errorbars for the black points are suppressed to avoid cluttering, but they are identical to the colored ones.
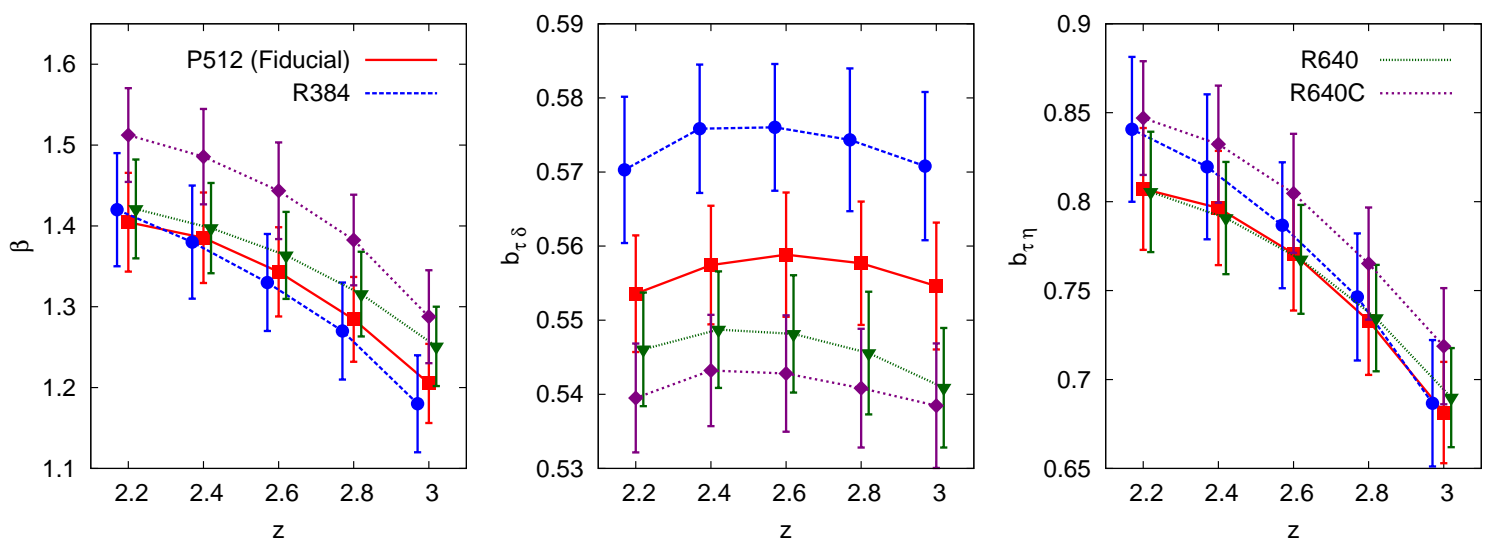

Figure 9. Redshift distortion parameter and bias factors fitted for three simulations of increasing physical resolution: R384, fiducial, and R640. These three models all have a $512^{3}$ grid, and we compare them also to R640C, with a coarser grid of $256^{3}$ and the same particle number as R640. Blue circles for R384 are shifted slightly left, and green triangles for R640 slightly right, to avoid superposition of errorbars.

The bias factors derived from the $q_{2}=0$ fits are shown in figure 9 . The errors are the formal ones derived from the $\chi^{2}$ fits, but the more important errors on the biases are of a systematic nature arising from the limited size of our boxes, and the need to fit the curves of $P_{F} / P_{L}$ at small scales where the simulations provide much more information than at large scales. The results are fairly similar for all the models shown in this figure. The largest 
difference is the higher value of $\beta$ derived for R640C, but as discussed above, this does not reflect the true difference of power at low $k$ between R640C and R640 shown by the colored and black points in figure 8 , and is an artifact of our fit with $q_{2}=0$. The real systematic errors in the bias factors are better reflected by the difference with the fits with free $q_{2}$.

We conclude that the errors introduced by the limited resolution of the grid size are substantial, and that future work should study the convergence with both particles and grid size for safely obtaining results for $P_{F}$ to accuracies better than $10 \%$. We note that our L80 and L120 simulations are analyzed also with a $512^{3}$ grid, which for a larger box size implies a lower physical resolution, with an expected impact on the power spectrum and bias factors following that seen in figures 8 and 9 .
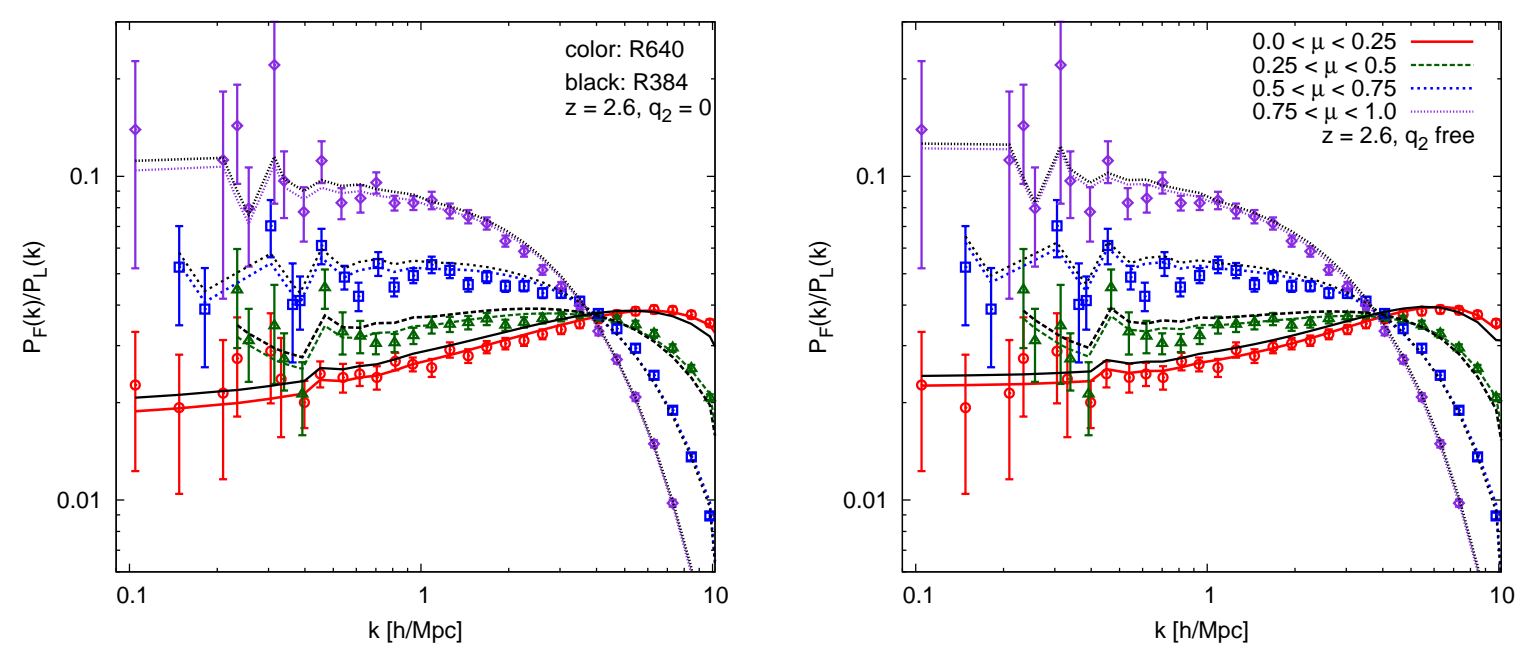

Figure 10. Comparing the power spectrum of simulations R640 (colored curves and points) and R384 (black curves, points omitted), at $z=2.6$, with higher and lower resolution compared to the fiducial model, respectively. Left panel: fits with $q_{2}=0$. Right panel: fits with free $q_{2}$.

Finally, we compare simulations of different resolution, varying the number of SPH particles. The simulations R640 and R384, which are the same as the fiducial one but varying the number of particles to $640^{3}$ and $384^{3}$, respectively, produce results for $P_{F} / P_{L}$ that are shown in figure 10 at $z=2.6$. In general, the changes due to the physical resolution are comparable to those caused by the resolution of the spatial grid but of opposite sign: decreasing the particle resolution decreases the power at high $k$, although in this case this is clear only for low $\mu$. In addition, the power at low $k$ generally drops with increasing resolution. In this case, we show in the left panel the two fits with $q_{2}=0$, and in the right panel the two fits with free $q_{2}$. The fit at low $k$ follows the points better for free $q_{2}$, as expected because of the extra degree of freedom, and the difference between the curves also better reflects the differences in the points for R640 and R384. However, as discussed before, it is not clear from the present simulations if the fits with free $q_{2}$ (which have a lower value of $q_{1}$ and therefore converge faster to the linear solution) are a more reliable result for the bias factors and the non-linear correction $D(k, \mu)$ at low $k$.

The derived bias factors for $q_{2}=0$ are also shown for both models in figure 9 . The bias factors for free $q_{2}$ are not shown, but are similar to those of the fiducial model shown below in figure 14. In general the density bias factor decreases by $\sim 5 \%$ when the particle resolution is doubled at fixed grid size, and increases by a smaller amount when the grid resolution is 
doubled. We note here that the changes at low $k$ induced by the small-scale resolution effects are due to non-linear couplings that depend on the fact that we always keep a fixed value of $\bar{F}$; the result would in general be different if instead we kept a fixed value of the ionizing background intensity.

We therefore conclude that the limited resolution and grid size in our simulations affect the values of the bias parameters by $\sim 5 \%$, and unfortunately our results do not show yet a clear convergence with the resolution. Increasing the number of particles or the number of grid cells modifies the results in opposite directions. Higher resolution simulations will be needed to better understand the conditions for convergence and to obtain more accurate results for the non-linear power $P_{F}(k, \mu)$ and the bias factors. Nevertheless, our derived values of the bias factors and $\beta$ at any redshifts vary by less than $10 \%$ in all of our simulations, and the predicted non-linear shape is also not subject to greater relative changes arising from resolution, so we believe that our results are reliable within this level of uncertainty.

Our results on the sensitivity to the simulation resolution can be compared to those obtained in [35]: their figure 12 shows the quantity $\Delta_{F}^{2}=k^{3} P_{F} /\left(2 \pi^{2}\right)$ (equal to what we plot below in figure 19). In agreement with our results in figure 10, lower resolution decreases the power at high $k$ and increases it at low $k$, and these changes are more pronounced at low $\mu$. However, the sensitivity to resolution seems to be weaker in our SPH simulations. Our R640 and R384 simulations have an interparticle separation that is about twice the cell size of the $L 10_{N} 128$ and $L 10_{N} 256$ simulations of [35], and yet the differences between the two simulations are $\sim 40 \%$ in [35], and only $\sim 10 \%$ between R640 and R384 in our case. The likely explanation for this is that the value of $\sim F$ is not held fixed when comparing simulations of different resolution in [35], and that our results shown at $z=2.6$ are mostly sensitive to gas densities above the mean density of the universe, where the resolution of particle-based codes improves over that of Eulerian codes. At higher redshift, however, the Ly $\alpha$ forest probes lower overdensities and the resolution effects in our simulations probably become more severe.

\subsection{Simulation Variance and Box Size}

The limited box size of any cosmological simulation implies the presence of a statistical error and a systematic error on any quantities that are measured from the simulation, such as the power spectrum of any tracer. The statistical error is due to the shot noise arising from the finite number of structures of a given scale that are formed within the simulation volume; if a number $N_{\text {sim }}$ of simulations with the same box size are performed with different random initial conditions, this statistical error is reduced as $N_{\text {sim }}^{-1 / 2}$. The systematic error, which is not reduced by averaging over several simulations, is due to the discretization of Fourier modes, which need to have cartesian components on the box axes that are multiples of $k_{1}=2 \pi / L$, and the absence of any modes below the wavenumber $k_{1}$. We therefore start by looking at the statistical variations of the power spectrum in the fiducial model, with $L=60 h^{-1} \mathrm{Mpc}$, by examining three simulations run with three different random seeds for the initial conditions: Seed 1 is for our fiducial model shown in previous figures, Seed 2 is for independent initial conditions where the Fourier mode amplitudes are generated with the correct Rayleigh distribution, and Seed 3 for independent initial conditions varying only the Fourier mode phases of Seed 1, but keeping the square amplitudes fixed to the mean value determined by the matter power spectrum, as in Seed 1 (see §3.1.1).

Figure 11 shows $P_{F} / P_{L}$ at $z=3$ for the first two seeds as points, and the fits with $q_{2}=0$ as curves. Seed 2, with the Rayleigh distribution, is shown as colored curves and points, and 


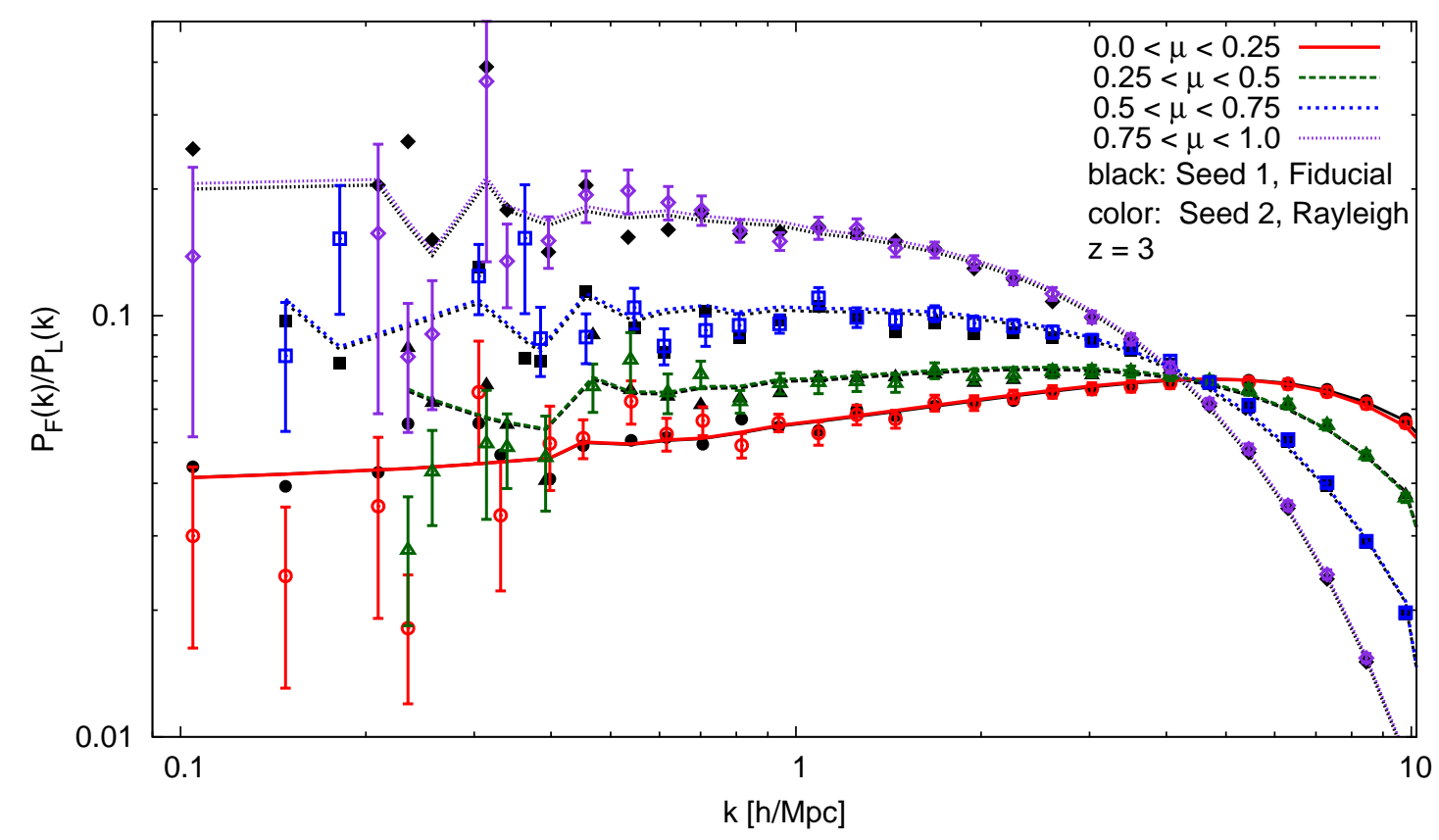

Figure 11. Comparison of $P_{F} / P_{L}$ for two different random initial conditions for the fiducial simulation at $\mathrm{z}=3$, with a $60 \mathrm{Mpc} / \mathrm{h}$ box size. Seed 1 is our usual fiducial simulation (black curves and points with errorbars omitted), with all Fourier mode square amplitudes fixed to the mean value predicted by the matter power spectrum of the model. Seed 2 is for different initial conditions with the Rayleigh distribution of amplitudes (colored curves and points with errorbars).
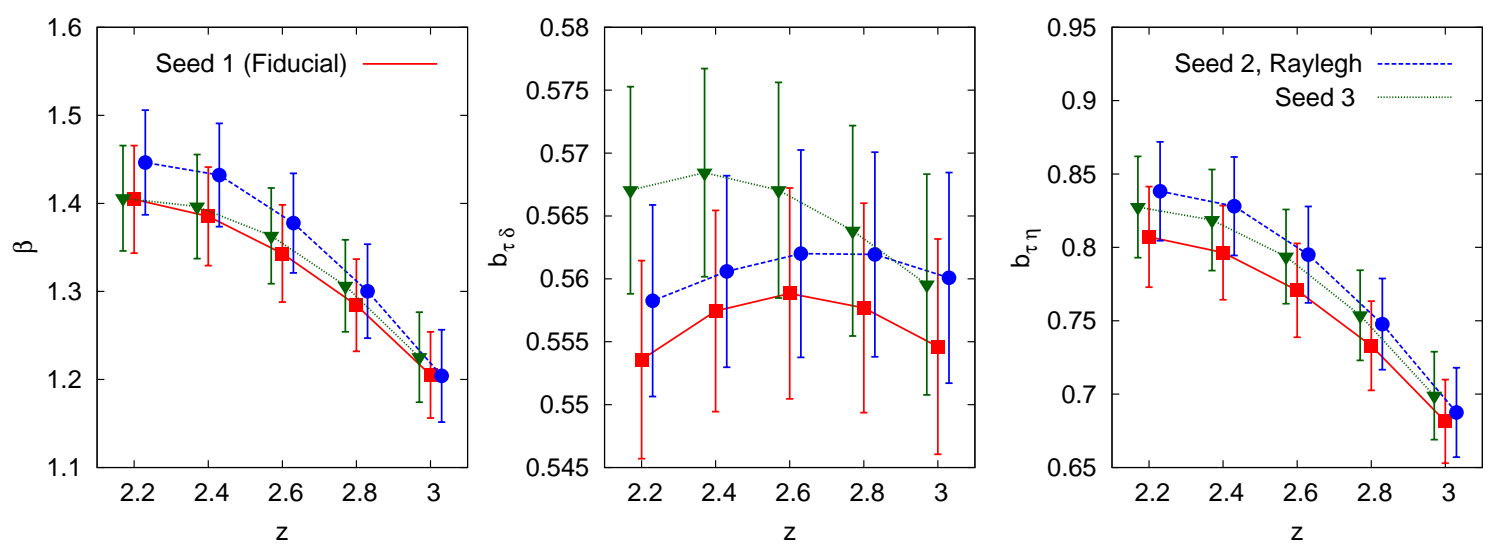

Figure 12. Comparison of the redshift distortion parameter and linear bias factors for three different random initial conditions for the fiducial simulation, obtained from the fits with $q_{2}=0$.

our usual fiducial simulation (Seed 1) is the black curves and points. The difference in the two fits is very small, the largest difference occurs at low $k$ and high $\mu$ and is only $\sim 3 \%$. This is despite the large random differences seen at low $k$ due to the introduction of random amplitudes for seed 2 . The fit does not have much freedom to vary owing to the large number of modes at small scales, resulting in very small variations for the high- $k$ points between the two simulations, so the large scatter at low $k$ gives small variations in the derived bias factors. 
Despite this, we see that the fits are generally good for both seeds. At $z=3$, the $q_{2}=0$ fits are in fact better at low- $k$ than the cases at lower redshift shown in previous figures, and are also closer to the free $q_{2}$ fits. Note that the even though the errorbars are shown around the measured simulation points, they need to be computed for the predicted $P_{F}$ of the fitted model, so points that are below the model are not as statistically discrepant as they look in the figure (e.g., fourth red circle and first green triangle starting from the left).

The derived linear bias factors are plotted in Figure 12 at all redshifts, for all three seeds, for the $q_{2}=0$ fits. Typically, the two bias factors can have random variations of up to $\sim 4 \%$ when varying the initial conditions, and this is not strongly affected by including the Rayleigh distribution of Fourier mode amplitudes. We have tested that these conclusions remain valid for an additional two independent seeds, not shown here. This uncertainty due to the random initial conditions is therefore comparable to the one due to the resolution of the simulations. These tests also show that the difference between including or not the Rayleigh distribution of mode amplitudes in the derived average $P_{F}$ is small enough for the purpose of the present study.

Next, we compare two simulations of the same model that differ only in the box size, but have the same physical resolution in both particles and grid cells: L120 and R384C. The R384C simulation has box size $L=60 h^{-1} \mathrm{Mpc}$, half of that of L120, and also half the number of particles and grid cells along a LOS, so that the initial interparticle spacing and grid cell size are the same. The ratio $P_{F} / P_{L}$ is shown in figure 13 for these two models at $z=2.2$. The upper panel shows the fits with free $q_{2}$, and the lower panel the fits with $q_{2}=0$. Two types of differences induced by the finite box size are seen in this plot. First, at low $k$, the accuracy in fitting bias factors is increased as the box size is increased and more points are available to determine the linear limit of the power spectrum. This is seen more clearly for the lowest $\mu$ curve, which is raised by $\sim 10 \%$ from R384C to L120 in the free $q_{2}$ fit. Second, the points for the $\mathrm{R} 384 \mathrm{C}$ simulation are generally lower than for L120 even at high $k$, with the largest difference ocurring also for the low $\mu$ curve, where it reaches $\sim 5 \%$. The main reason for this difference at high $k$ is that we are always computing the Ly $\alpha$ transmission power after fixing $\bar{F}$, and the value by which we need to multiply the optical depth to achieve a fixed $\bar{F}$ changes in the various models we show. The large-scale power that is missing in the $\mathrm{R} 384 \mathrm{C}$ simulation results in larger voids with lower densities, and for a fixed intensity of the ionizing background, this would result in a higher $\bar{F}$. The intensity of the ionizing background therefore needs to be lowered to have the same $\bar{F}$ in the two simulations, and this induces the change in power at high $k$. At the highest values of $k$ near $10 h^{-1} \mathrm{Mpc}$, the power measured in the two simulations shown by the colored and black points is very close, but as we have seen previously this power is quite sensitive to the particle and grid resolution, which are the same in these two simulations.

Linear bias factors are shown in figure 14 for the fits with free $q_{2}$ (blue circles for L120 and purple rhombi for R384C), where a third model of intermediate box size, L80, has been added (green triangles). The results for the fiducial model, on a $60 h^{-1} \mathrm{Mpc}$ box, are also shown as red squares (these were shown only for the $q_{2}=0$ fit in previous figures). In general, the bias factors are higher by $\sim 10 \%$ for the free $q_{2}$ fit compared to the $q_{2}=0$ fit. While the bias factors are nearly the same for L120 and R384C, the L80 simulation has a larger value of $b_{\tau \delta}$ by $\sim 5 \%$ at all redshifts, while $b_{\tau \eta}$ is nearly the same, and therefore $\beta$ is lower for L80. This may seem strange because L80 is a simulation of intermediate box size. The L80 simulation also has the same particle resolution as the others, but its grid size is smaller (with the same number $512^{3}$ of cells as the larger box of L120). As seen previously 

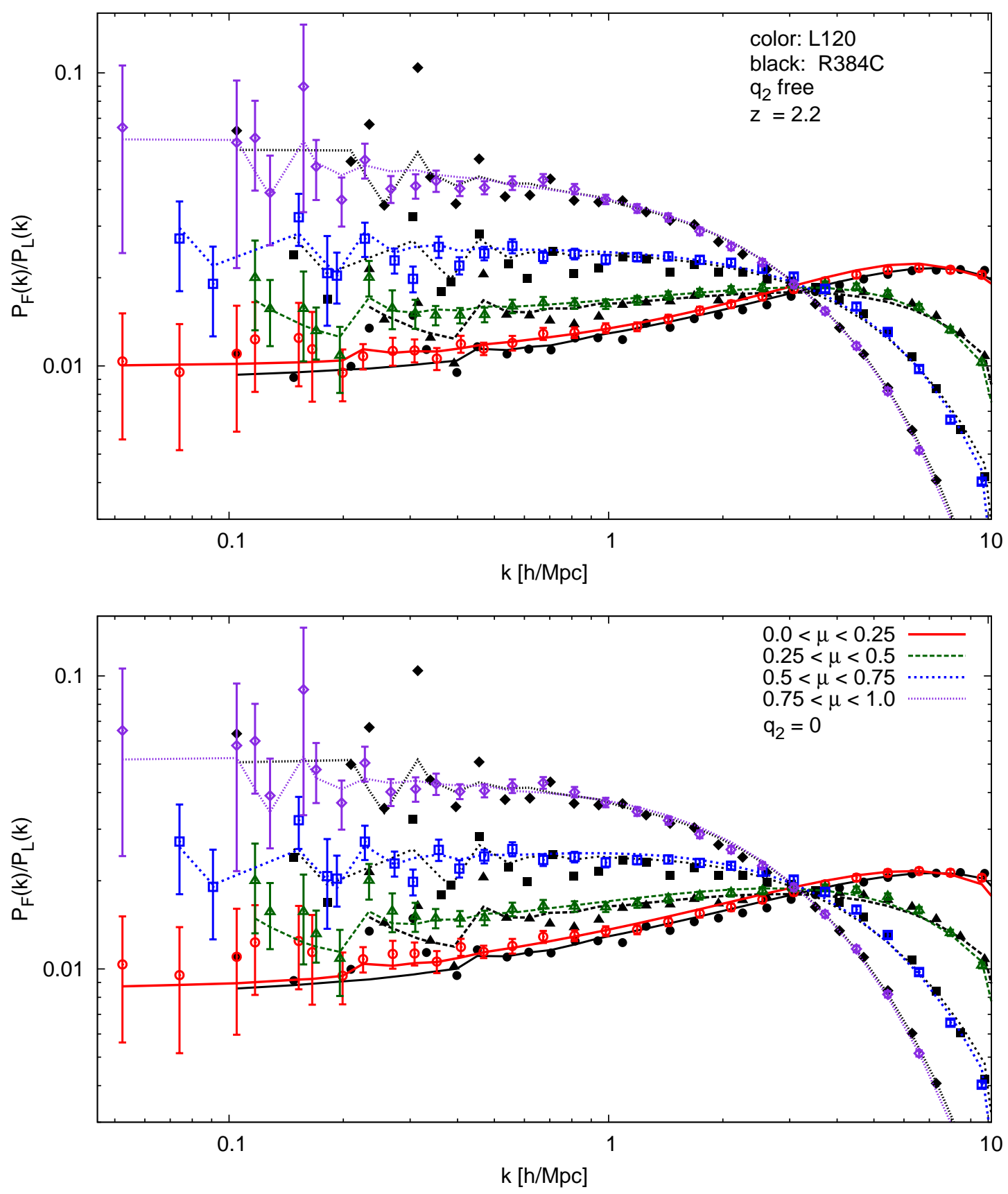

Figure 13. Comparison of $P_{F} / P_{L}$ from simulations L120, with box size of $120 \mathrm{Mpc} / h$ (colored points and curves), and $\mathrm{R} 384 \mathrm{C}$, on a $60 \mathrm{Mpc} / h$ box (black curves and points with omitted errorbars), at $z=2.2$. The two simulations have equivalent physical resolution, with $768^{3}$ and $384^{3}$ particles, and $512^{3}$ and $256^{3}$ grid cells, respectively, at $z=2.2$, so differences should be due to the effects of box size and the random initial conditions displaced by a factor of 2 in $k$ only. Upper panel shows the fit with $q_{2}$ as a free parameter, and bottom panel is for $q_{2}=0$.

in figure 9, increased grid resolution raises the value of $b_{\tau \delta}$ and lowers $\beta$, and correcting for this brings the green lines (for L80) a bit closer to the blue and purple lines (for L120 and 

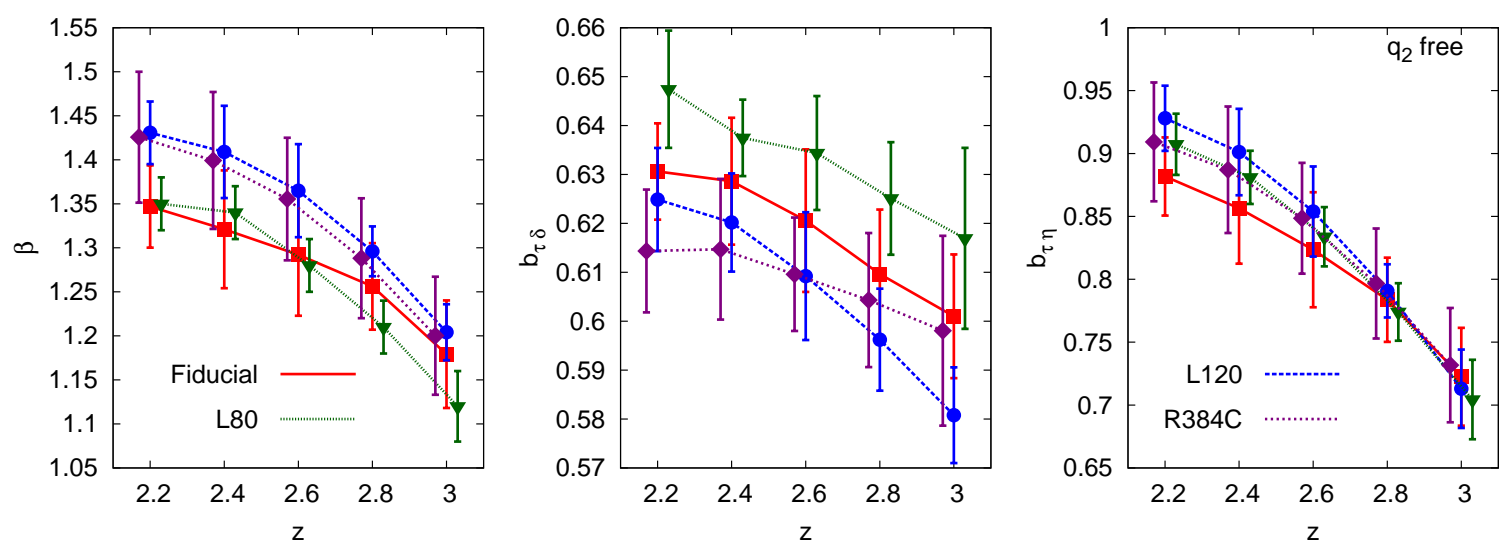

Figure 14. Comparison of the bias and $\beta$ parameters for different box sizes. Notice that while the R384C and L120 simulations have equivalent physical resolution, L80 has the same particle density but a different physical grid size. The results shown here are for free $q_{2}$ in the $D_{1}$ formula.

R384C) in figure 14. However, most of the difference remains, and we believe this can be assigned to the effects of random initial conditions. Even though these simulations have the same Rayleigh-suppressed initial conditions on modes of fixed $k L$, the power of a simulation at fixed $k$ still has random variations, and therefore we can expect some of the variations in bias factors analogous to the ones between seeds 1 and 3 seen in figure 12 .

We conclude that the systematic effects of the box size are comparable to the random variations due to different initial conditions seen in figure 12, and also to the systematics due to limited resolution in our simulations. If anything, the error resulting from using different fits to the whole shape of $P_{F} / P_{L}$, which we have illustrated by including or not including the additional free parameter $q_{2}$ in this paper, is a bit larger: both $b_{\tau \delta}$ and $b_{\tau \eta}$ are systematically larger by $\sim 10 \%$ for free $q_{2}$ than for fixed $q_{2}=0$ at low redshift in most of our models, with a smaller difference at high redshift. The better fit that is obtained for free $q_{2}$ to the low- $k$ points suggests this to be more reliable, but this fit also implies a rather small value of the $q_{1}$ parameter (see figure 5), and therefore a surprisingly fast convergence of the non-linear factor $D_{1}$ to unity at low $k$. This remains to be tested with more large box simulations.

\subsection{Comparing Lagrangian and Eulerian simulations}

As a final test, we analyze here a simulation that is directly run on an Eulerian grid instead of using gas particles. This is the simulation labeled "Euler" in table 3.1 and described in section 3.1.2. As a comparison, we have run an SPH simulation designed to match the physical parameters of the Euler simulation, so that a comparison can be made. This simulation is labeled "Lagrange" in table 3.1: it has the same box size of $L=50 h^{-1} \mathrm{Mpc}$, and resolution of $512^{3}$ both in number of particles and grid cells. The model mass fluctuation power spectrum is the same, and we have also attempted to match the two simulations to have the same density-temperature relation, which depends on the model of the ionizing background and the heating and reionization history. Unfortunately, it is not possible to do this match exactly owing to the different methods for computing heating in the two simulations. In addition, the initial conditions were not the same in the two simulations, (in this case both of them include the Rayleigh distribution of Fourier mode amplitudes), so this inevitably introduces some difference in the results. 


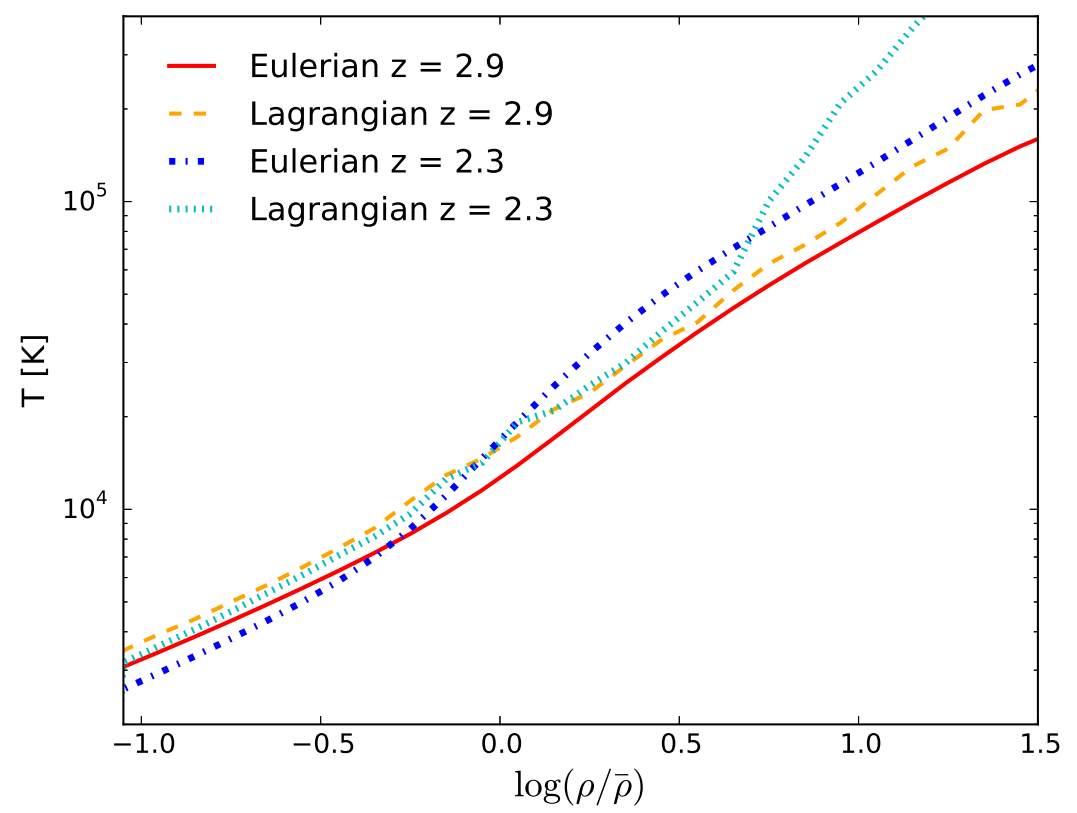

Figure 15. Mean temperature as a function of density for the Eulerian and Lagrangian simulations, at $z=2.3$ and $z=2.9$.

The values of the mean transmission for the Eulerian simulation were determined by the model of the ionizing background evolution used in that simulation, and are equal to the following values: $\bar{F}=0.8042,0.7484$ and 0.6725 at the three redshift outputs of $z=2.3,2.6$ and 2.9, respectively. We choose to rescale the optical depths in the Lagrange simulation to match these same values of the mean transmission, which are different from our standard ones used in previous figures. The redshift outputs for the simulation Lagrange are our standard set of 5 values, for which we set the mean transmission to the same value as the Eulerian one at $z=2.6$, and to values following a power-law dependence of the same form as in equation 3.1 , separately chosen to match the mean transmission of the Euler simulation for $z<2.6$ and for $z>2.6$. This results in the following values: $\bar{F}=(0.8212,0.7864,0.7484,0.6989,0.6452)$ at $z=(2.2,2.4,2.6,2.8,3.0)$.

The full density-temperature relation of the Euler simulation is shown in figure 16, at redshifts $z=2.3$ and $z=2.9$. The temperature that is shown is the average in all the cells that have the density in each bin of width $\Delta \log \rho=0.1$. The relation is not exactly a powerlaw, and it steepens above $\rho / \bar{\rho} \sim 1$ due to shock-heating of the gas in the sheets and filaments of the Ly $\alpha$ forest as structure formation develops. The two simulations are fairly close to each other, but in the low-density regime the Eulerian one has lower temperature by $\sim 0.1$ dex. At densities $\rho / \bar{\rho}>1$ the shock heating in the Eulerian simulation is higher than in the Lagrangian one, particularly at low redshifts, and at $\rho / \bar{\rho}>5$ the Lagrange simulation shows more heating at low redshift. This seems to be affected by the collapse and sudden heating of a few clusters in the simulation and is therefore affected by random variance depending on initial conditions. We have obtained a linear regression fit to the $\log T-\log \rho$ relation of the Euler simulation in the low-density regime $-1.5<\log (\rho / \bar{\rho})<-0.3$, in which shock heating is not important, with the result indicated in Table 3.1 for $z=2.6$, which has a weak variation with redshift. The difficulty in obtaining a more precise match of the density- 


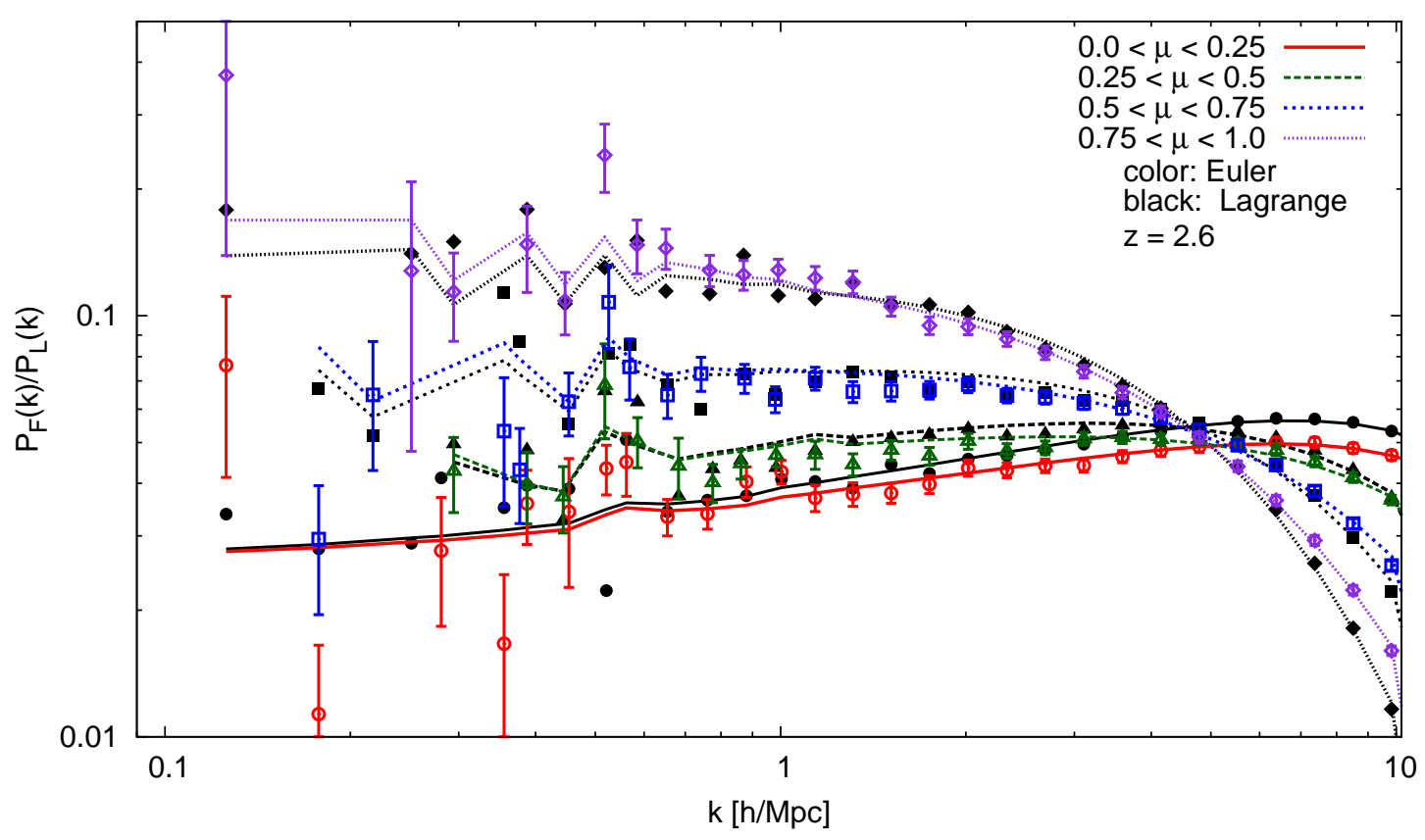

Figure 16. Comparison of $P_{F} / P_{L}$ for the Euler and Lagrange simulations, using an Eulerian code and the GADGET-II code, of the same cosmological model, at $z=2.6$.
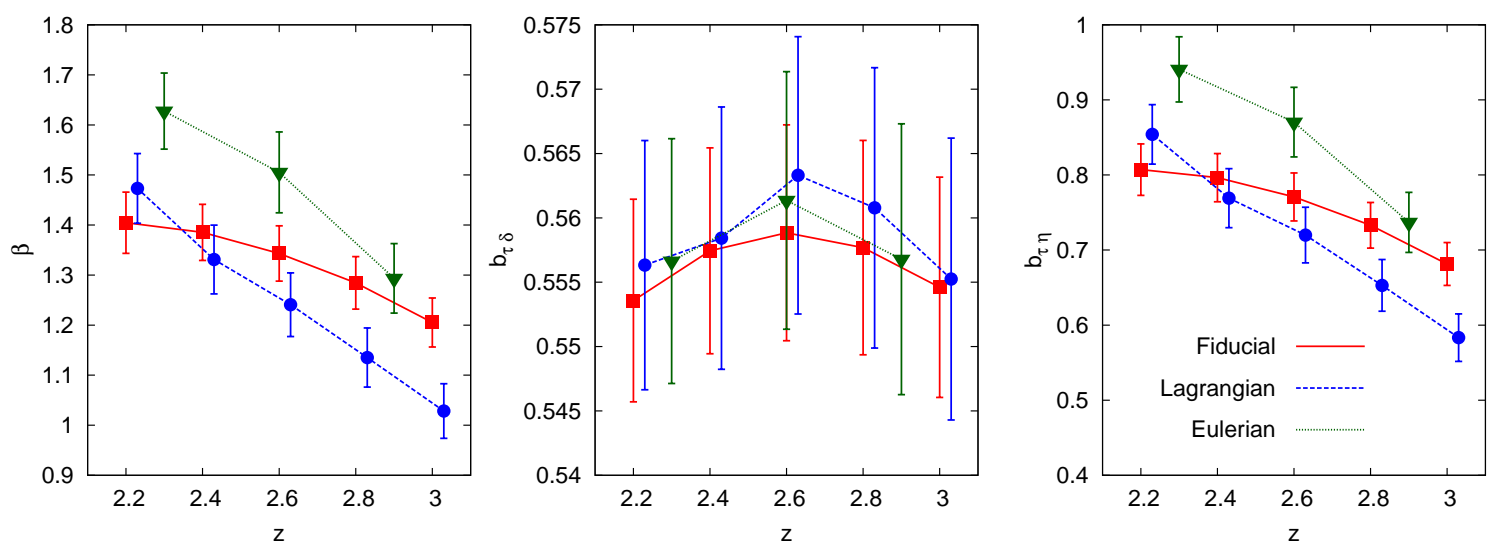

Figure 17. Redshift evolution of $\beta$ and the bias factors for the Euler and Lagrange simulations, from the fits with $q_{2}=0$.

temperature relation in the Eulerian and Lagrangian simulations illustrates the problems that are encountered when testing that numerical simulations using different codes and different methods make the same predictions for the Ly $\alpha$ forest power spectrum. More precise tests, that start from exactly the same initial conditions and force the same thermal histories, should be performed in order to investigate the differences between the two frameworks more completely (see, e.g., [50], where a comparison between SPH and Eulerian codes in terms of the $1 \mathrm{D}$ transmission power spectrum is presented and where differences of order $<5 \%$ are found).

The ratio $P_{F} / P_{L}$ is shown in figure 16, for the Euler simulation as colored curves and 
points, and the Lagrange one for black curves and points with omitted errorbars, at $z=2.6$. The curves are fits to the $D_{1}$ formula with $q_{2}=0$. Important differences between the two simulations are apparent: first, at low $k$, the high- $\mu$ curves are higher for the Euler simulation, implying a higher predicted value of $\beta$. At high $k$, the curve at lowest $\mu$ is lower for Euler than Lagrange, whereas the high- $\mu$ curves are also at lower power except at $k>5 h^{-1} \mathrm{Mpc}$, where the power decreases more slowly for Eulerian simulation. The differences are clear and at the level of $\sim 10 \%$, and they may be due to several effects having to do with the different resolution and methods of the simulations. It is useful in particular to compare with figure 10, which shows that poor resolution produces a sharper decline of the power at the highest $k$, suggesting that the poorer resolution of the Lagrange simulation compared to the Euler one at mean density can explain this difference in figure 16. Nevertheless, the fact that the two simulations agree within $10 \%$ on the predicted power (except at low $k$ and high $\mu$ where the difference grows to $20 \%$, but is sensitive to random variance from the different initial conditions) is reassuring.

The bias factors are shown in figure 17. The redshift distortion factor (left panel) predicted by the Euler simulation is substantially higher than for the other simulations analyzed so far, corresponding to the higher power of the colored curve at low $k$ and high $\mu$ in figure 16 , whereas the variations in $b_{\tau \delta}$ are very small, but as discussed above this is sensitive to the variance in the different initial conditions. The differences in the predictions between the two methods will need to be understood in more detail before theoretical predictions can be made from these hydrodynamic cosmological simulations at a high level of accuracy. However, the main features of the evolution of these bias factors are common to all our results: $b_{\tau \delta}$ is roughly constant with redshift and near $60 \%$, and $b_{\tau \eta}$ is slightly below unity and declines with redshift, implying also a decline of $\beta$ with redshift.

\section{Dependence of the $\operatorname{Ly} \alpha$ Power Spectrum on the Physical Model}

We analyze in this section the dependence of the Ly $\alpha$ transmission power spectrum on the physical characteristics of the IGM and the cosmological model. As described in $\S 1$, the Ly $\alpha$ power spectrum depends mainly on the amplitude and shape of the initial matter power spectrum, the density-temperature relation, and the value of the mean transmission at each redshift. Other properties of the cosmological model should not affect $P_{F}(k, \mu)$, except for rescalings: for example, changes in $H_{0}, \Omega_{b}$ and $\Omega_{m}$ simply imply a rescaling of the Jeans scale and any physical scale in the power spectrum in terms of angular and redshift separations, which are the observed coordinates of spectral pixels. The details of the gas cooling time, which depend on $\Omega_{b}$ and the ionizing background spectrum, can be thought of as being incorporated in the density-temperature relation and its history, which determine the impact of the Jeans scale and the thermal broadening on the Ly $\alpha$ power spectrum.

The dependence of the Ly $\alpha$ power spectrum with the most relevant physical parameters are described below, by examining the following cases:

- Dependence on the amplitude of the power spectrum, characterized by the parameter $\sigma_{8}$, which we vary for our fiducial model $(\S 6.1)$.

- Dependence on the power spectrum slope on the range of scales examined by our simulations, which we illustrate by comparing the fiducial and Planck models ( $(6.2)$.

- Dependence on $\bar{F}$ at a fixed redshift, and redshift evolution ( $\S 6.3)$. 
- Dependence on the density-temperature relation, parameterised as $T=T_{0}(\rho / \bar{\rho})^{\gamma-1}$ $(\S 6.4)$.

Throughout this section, we use only fits with the formula $D_{1}$ fixing $q_{2}=0$.

\subsection{Power spectrum amplitude}
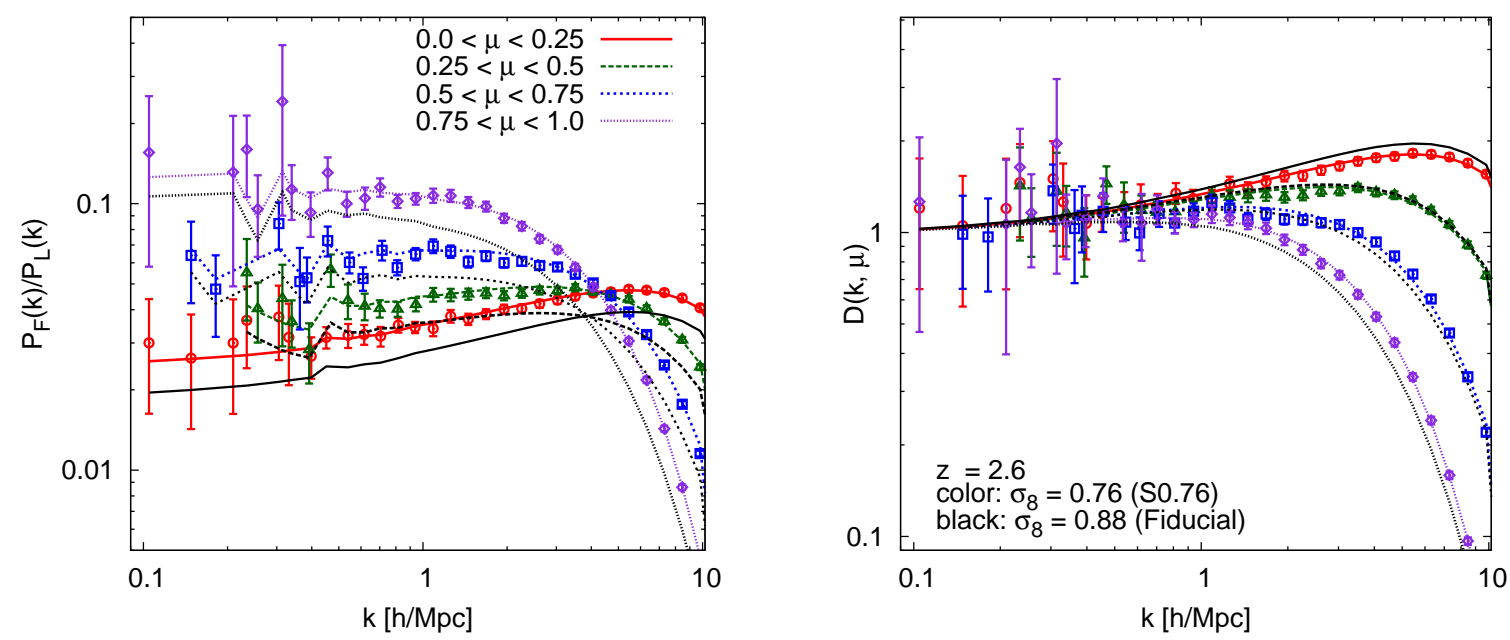

Figure 18. Comparison of $P_{F} / P_{L}$ (left panel) for models with different power spectrum amplitude, S0.76 with $\sigma_{8}=0.7581$ (colored curves and points), and the fiducial model with $\sigma_{8}=0.8778$ (black curves; points omitted), at $\mathrm{z}=2.6$. The non-linear term $D(k, \mu)$ is shown in the right panel. As expected, $D-1$ increases with the amplitude, and the characteristic scale at which non-linear anisotropy starts dominating, where curves with different $\mu$ cross each other in the left panel, increases $\left(k_{n a}\right.$ decreases) with the amplitude.

The ratio $P_{F} / P_{L}$ is compared in figure 18 (left panel) for the model S0.76 and the fiducial model. The only difference between these two models is in the amplitude of their linear power spectrum, which is lower by a factor $(0.7581 / 0.8778)^{2}=0.746$ in S0.76. The colored curves are generally higher than the black ones by a factor that is slightly smaller than 0.746 , indicating that $P_{F}$ increases slowly with $\sigma_{8}$, so $P_{F} / P_{L}$ decreases although not as fast as $\sigma_{8}^{-2}$. As the power spectrum amplitude increases, we expect that non-linear effects become important at increasingly long scales. This is confirmed by the fact that the characteristic wavenumber $k_{n a}$ at which the curves with different $\mu$ cross each other decreases with $\sigma_{8}$. This crossing point represents the characteristic scale where the power spectrum quadrupole changes sign, switching from the linear Kaiser effect to the non-linear velocity dispersion effects. In the right panel, the non-linear correction $D(k, \mu)$ is shown, which generally increases its departure from unity as $\sigma_{8}$ is increased. Black points are omitted in these plots to avoid excessive cluttering, but they are similarly well fitted by the black curves as for the colored ones.

The direct dependence of $P_{F}$ on the amplitude is better visualized in figure 19, where we plot the quantity $P_{F}(k) k^{3} /\left(2 \pi^{2}\right)$ for the same models as in figure 18 . Analogously to the amplitude $\Delta^{2}(k)$ in equation (3.5), this is the dimensionless Fourier amplitude of transmission fluctuations. Here the black points are also included (with omitted errorbars, which are equal to the colored errorbars). At low $k, P_{F}$ increases very little for $\mu=0$ as $\sigma_{8}$ increases, but this increase is more pronounced at high $\mu$, indicating an increase of $\beta$ with $\sigma_{8}$. At high $k$, 


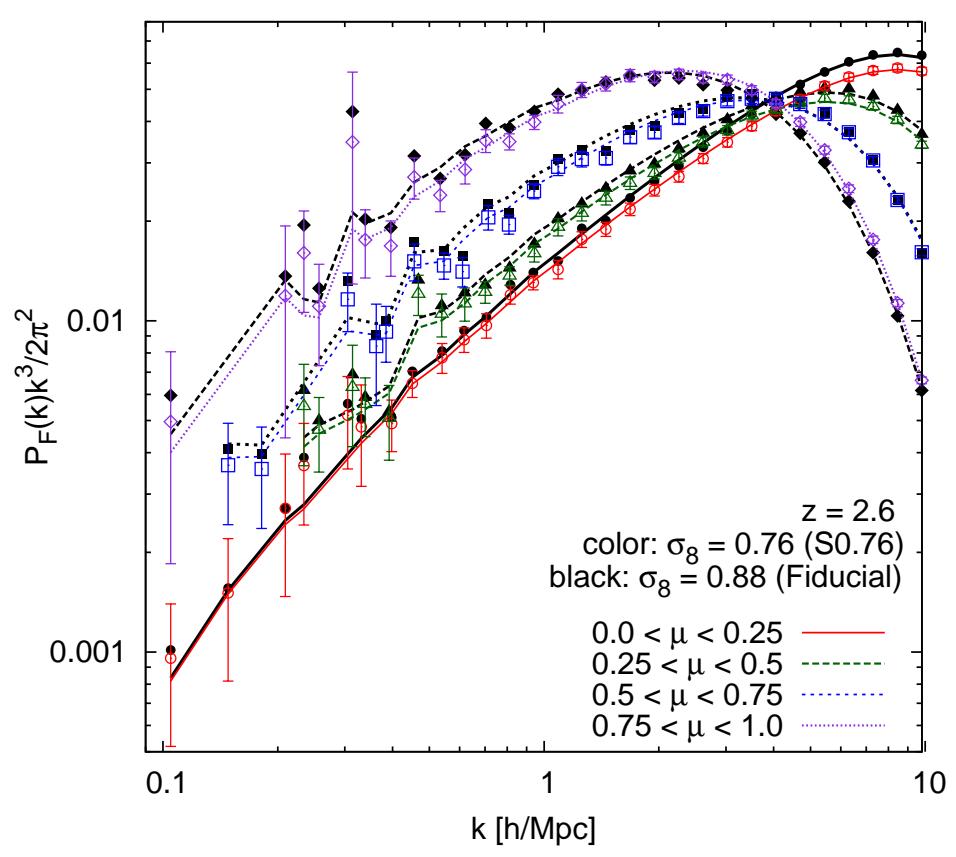

Figure 19. Amplitude of transmission fluctuations in Fourier space, defined as $P_{F} k^{3} /\left(2 \pi^{2}\right)$. The power $P_{F}$ increases slowly with the amplitude of the linear power spectrum parameterized by $\sigma_{8}$, except at high $k$ and $\mu$ where it decreases with $\sigma_{8}$. Black points, for the fiducial model, have their errorbars omitted but they are equal to the colored ones.

$P_{F}$ continues to increase with the linear amplitude at low $\mu$, but it decreases at high $\mu$. This corresponds to the decrease of the wavenumber $k_{n a}$ for the crossing of the curves in figure 18 with increasing $\sigma_{8}$.
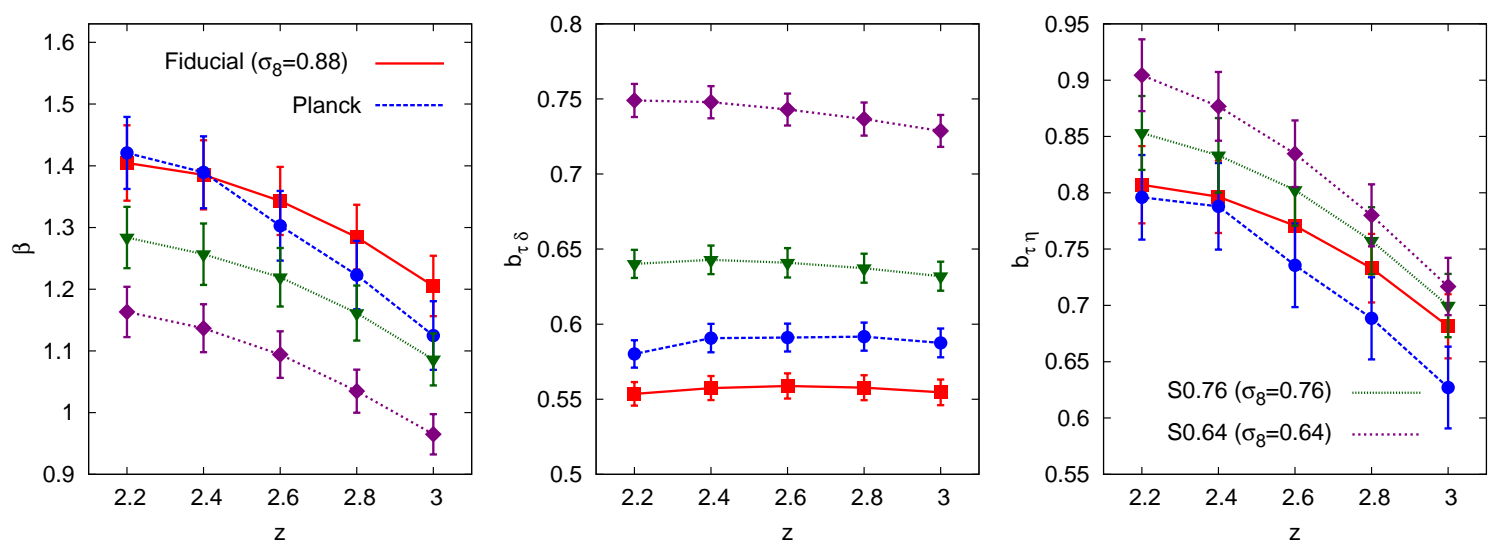

Figure 20. Bias and redshift distortion factors as a function of redshift for models S0.76 and S0.64, which differ from the fiducial one only for a lower linear amplitude of the mass power spectrum, and of the Planck model, which also has a different power spectrum slope. All values are obtained from the fits with $D_{1}(k, \mu)$ fixing $q_{2}=0$.

The change of bias and redshift distortion factors is shown in figure 20. The very weak dependence of $P_{F}$ on the linear amplitude at low $k$ and low $\mu$ implies that $b_{\tau \delta}$ decreases with 
the amplitude nearly as fast as $\sigma_{8}^{-1}$. At high $\mu$ the power $P_{F}$ has a substantial increase with $\sigma_{8}$, so the bias factor $b_{\tau \eta}$ decreases more slowly with $\sigma_{8}$, particularly at high redshift, and as a consequence $\beta$ increases with $\sigma_{8}$, as seen in the left panel.

\subsection{Power spectrum slope}
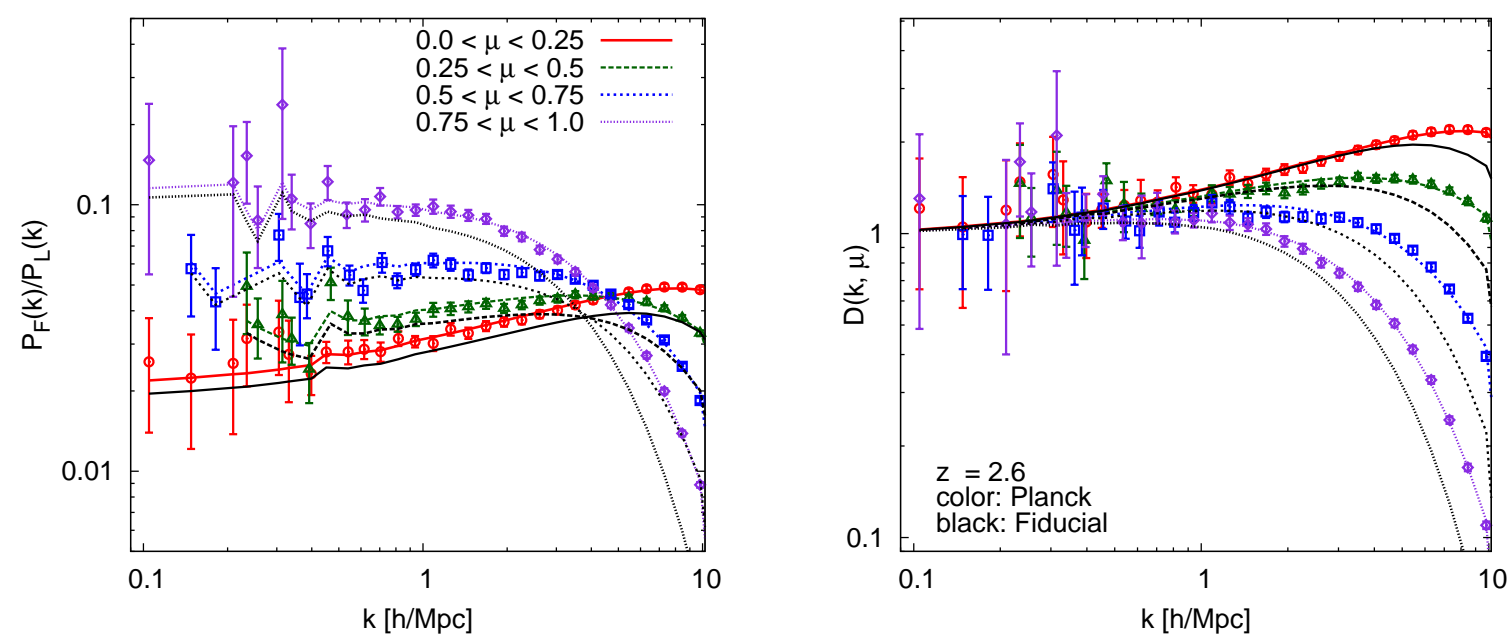

Figure 21. Comparison of $P_{F} / P_{L}$ (left panel) for the fiducial model (with $n_{s}=1$ ) and the Planck model (with $n_{s}=0.9624$ ).

We now compare the model labeled as "Planck" in table 3.1, which has parameters consistent with the ones measured by the Planck mission, with the fiducial model. Figure 24 shows $P_{F} / P_{L}$ and $D(k, \mu)$ for the Planck model as colored curves and points, compared to the fiducial model as black curves (with points omitted). The transmission fluctuation amplitude, $P_{F}(k) k^{3} /\left(2 \pi^{2}\right)$, is shown also in figure 22 , in all cases at $z=2.6$. Interpreting the differences between these two models is in this case more complicated than in the previous subsection, because now both the amplitude and slope of the power spectrum are different. In addition the density-temperature relation also differs slightly for these two models as specified in table 3.1 .

The fiducial and Planck models have slightly different values of $\Omega_{0 m}$, implying a difference in their growth factors. It is therefore useful to express the mass fluctuation amplitudes on spheres of radius $8 h^{-1} \mathrm{Mpc}$ at $z=2.6$, instead of the present epoch, which have the following values: $\sigma_{8}(z=2.6)=(0.3102,0.2910,0.2679)$ for the fiducial, Planck and S0.76 models, respectively. The Planck model therefore has a linear amplitude that is close to the average of the fiducial and S0.76 models at the scale of $\sigma_{8}$. In addition, comparing figures 19 and 22, we can see that the amplitude of $P_{F}$ of the Planck model is intermediate between that of the fiducial and S0.76 models at the lowest values of $k$ probed by our simulations, $k \sim 0.2 h / \mathrm{Mpc}$. This value of $k$ roughly corresponds to the scale at which the parameter $\sigma_{8}$ measures the amplitude of density fluctuations, and can therefore act as an approximate pivot scale for comparing amplitudes of models with different slope. In agreement with this, the Planck model has a bias factor $b_{\tau \delta}$ intermediate between the values for the fiducial and S0.76 models (see figure 20). This bias factor has therefore little sensitivity to the power spectrum slope, and depends mostly on the amplitude parameter $\sigma_{8}(z)$. We caution, however, that this bias factor may also depend on the temperature-density relation, and is sensitive to 


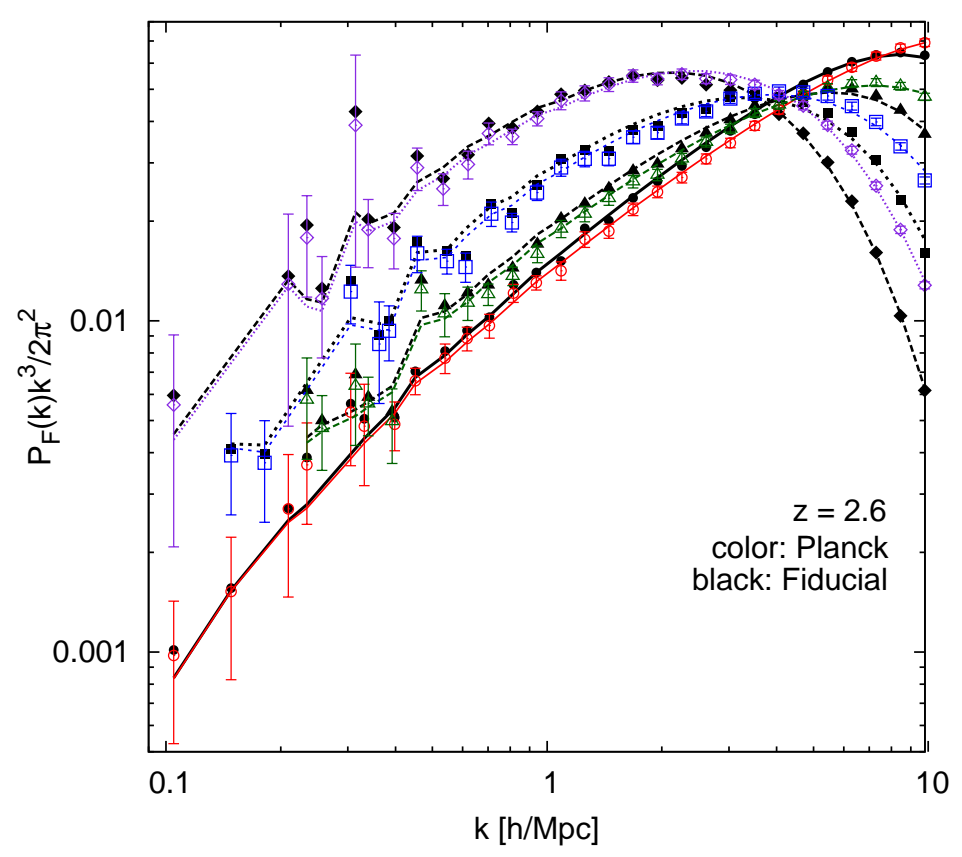

Figure 22. Amplitude of transmission fluctuations for the Planck model (colored curves and points) and the fiducial model (black curves and points with errorbars omitted).

the value of $\bar{F}$. The behavior of $b_{\tau \eta}$ and $\beta$ shows a steeper redshift evolution of the Planck model compared to the fiducial one with varying amplitude. This different evolution may be affected by the lower temperature of the Planck model compared to the fiducial one, as will be discussed in $\S 6.4$.

The ratio of the linear power spectra for the Planck model to that of the fiducial model varies approximately as $k^{-0.03}$ over the range of scales we explore, as caused mostly by the difference in the primordial spectral index $n_{s}$ (with a smaller effect arising from the different value of $\Omega_{0 \mathrm{~m}} h$ ). One might expect a similar $k$-dependence of the ratio of the transmission power $P_{F}$ for the two models, seen in figure 22 , but this would generally be misleading. In fact, figure 19 shows that the ratio of $P_{F}$ for the S0.76 and fiducial models has a similar $k$-dependence at low $\mu$, even though these two models have exactly the same $P_{L}(k)$ except for the normalization. This is caused by the increase of the non-linear correction $D$ with $\sigma_{8}$ at low $k$ and $\mu$.

In general, the sensitivity of $P_{F}$ to $P_{L}$ is relatively weak on scales $k \gtrsim 0.3 h^{-1} \mathrm{Mpc}$, when non-linear effects start. This means that $P_{F} / P_{L}$ tends to behave inversely with $P_{L}$, as found in $\S 6.1$ when analyzing the dependence of $P_{F}$ on the amplitude, and therefore to increase faster with $k$ for the Planck model compared to the fiducial one. Of course, on very large scales the constancy of the bias factors implies that $P_{F}$ is proportional to $P_{L}$ for a given model. At the same time, on very small scales $\left(k \gtrsim 3 h^{-1} \mathrm{Mpc}\right)$, the reason for the slower decline of $P_{F} / P_{L}$ for the Planck model compared to the fiducial one is caused mostly by the lower temperature in the Planck simulation (see $\S 6.4$ ).

Finally, figure 23 shows the non-linear parameters of the function $D_{1}(k, \mu)$ obtained in our fits for the fiducial, S0.76, S0.64 and Planck models. The parameter $k_{p}$ is mostly dependent on the gas temperature and is higher for the Planck model owing to its lower 

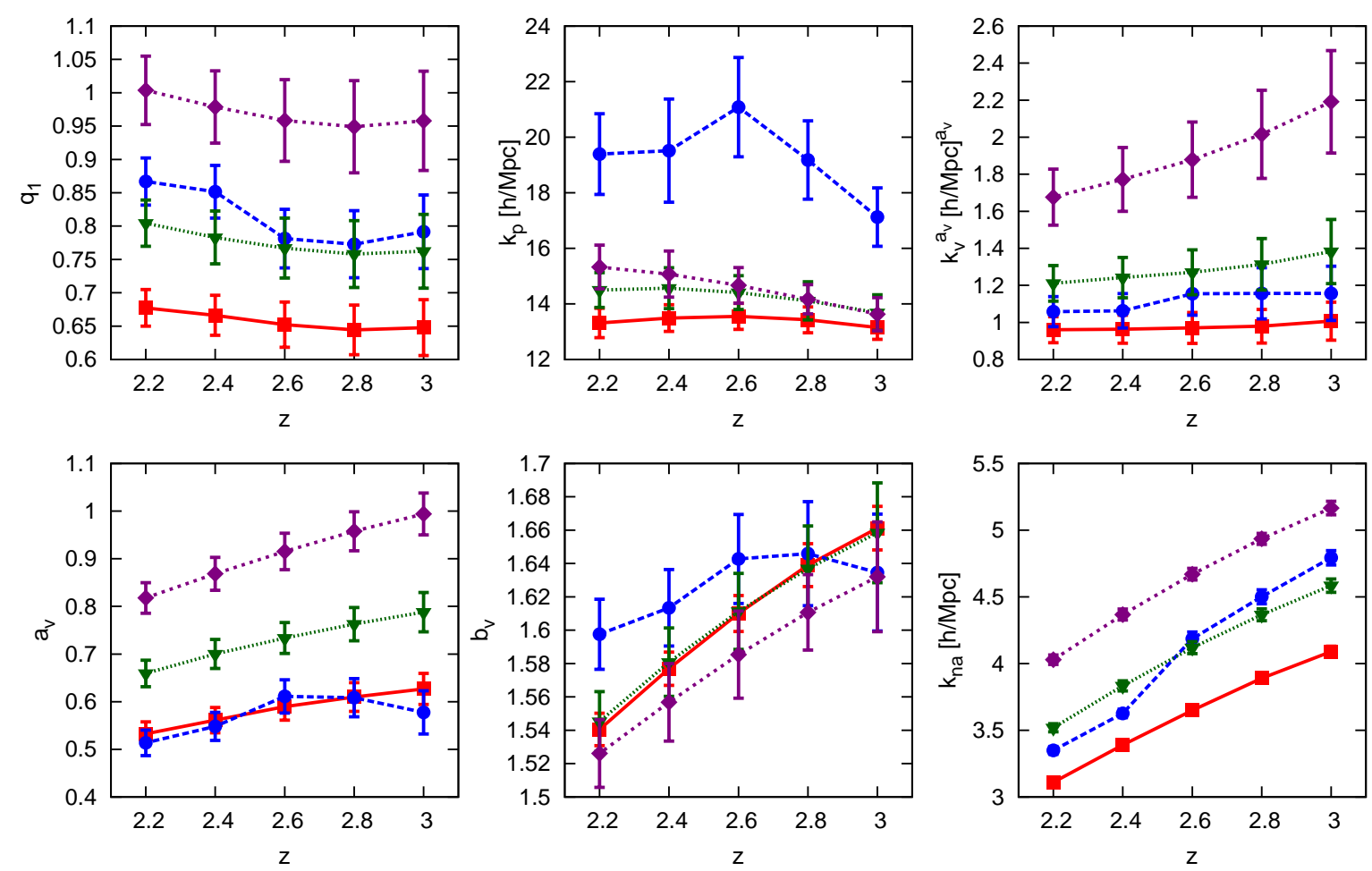

Figure 23. Parameters of the non-linear function $D_{1}(k, \mu)$ for the fiducial model (red squares), for the S0.76 and S0.64 models with a decreasing amplitude of the linear power spectrum (green triangles and purple rhombi), and for the Planck model, with an intermediate amplitude between the fiducial and S0.76 models and a lower $n_{s}$ (blue circles). The sixth panel shows the parameter $k_{n a}$, the wavenumber at which curves of different $\mu$ cross each other in the diagram of $P_{F}(k, \mu) / P_{L}(k)$, computed from equation (4.4).

temperature, and very similar for the other three models. The parameters $q_{1}, a_{v}$ and $k_{v}^{a_{v}}$ mostly follow a progression depending on the amplitude of $P_{L}$ : as the amplitude increases, $q_{1}$ is decreased because the impact of non-linear terms does not increase as fast as $\Delta^{2}(k)$ in equation (3.6), and $a_{v}$ and $k_{v}^{a_{v}}$ are also reduced. Variations of $b_{v}$ are rather small. The sixth panel in the figure shows $k_{n a}(z)$, representing the scale at which the curves of $P_{F} / P_{L}$ at different $\mu$ cross each other. As expected, the errors of this parameter are small because most of the degeneracy with other parameters is removed. The variation with redshift and $\sigma_{8}$ confirms what we have described: as the power amplitude increases, $k_{n a}$ decreases.

\subsection{Mean transmission fraction and redshift evolution}

The observed evolution with redshift of the non-linear power spectrum is a consequence of two different effects: the physical evolution that would be observed at fixed $\bar{F}$, and the change in $P_{F}$ that occurs when varying $\bar{F}$ at a fixed redshift. These two effects are clearly separated in figure 24 , where the change from $z=2.2$ to $z=3$ for $P_{F} / P_{L}$ is shown for the fiducial model at fixed $\bar{F}$. In the left panel, we choose the value of $\bar{F}$ that corresponds to $z=2.2$, and the standard result for $z=2.2$ for the fiducial model is shown as the black curves. Colored curves and points show the result at $z=3$ when using the value of $\bar{F}$ that corresponds to $z=2.2$. Similarly, the black curves in the right panel show our standard 

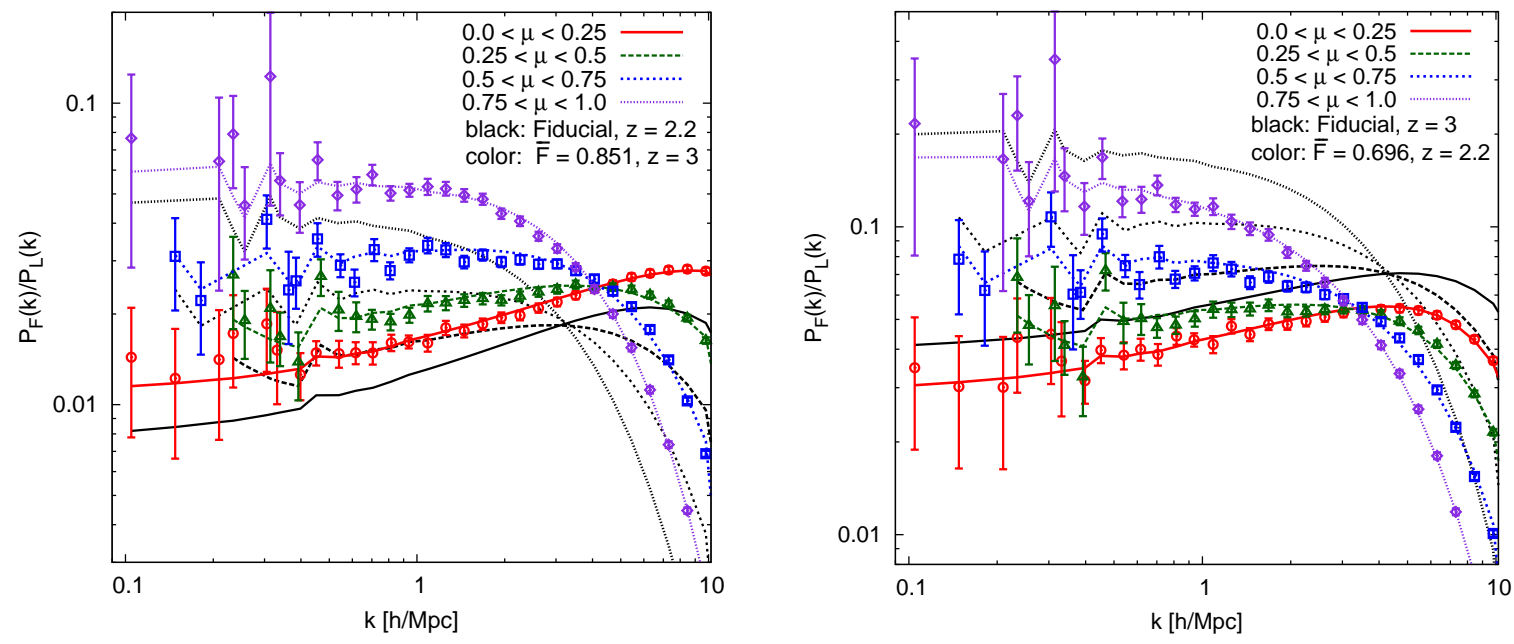

Figure 24. Evolution of $P_{F} / P_{L}$ with redshift when the mean transmission is kept fixed at $\bar{F}=0.851$ (left panel) and $\bar{F}=0.696$ (right panel). The result of the fiducial model is shown in both panels at $z=2.2$ and $z=3$, as black curves (with points omitted) when the true value of $\bar{F}$ is used, and as colored curves and points when $\bar{F}$ is modified to the value at the other redshift. The observed evolution, from the black curves in the left panel to those in the right panel, is a result of both the physical redshift evolution at a fixed $\bar{F}$, and the variation of $\bar{F}$ with redshift.

result at $z=3$, while the colored curves and points show the $z=2.2$ result for the value of $\bar{F}$ that corresponds to $z=3$. The density-temperature relation of our models is nearly constant with redshift, so this should have a minimal impact on the variations seen in this figure.

The redshift evolution at fixed $\bar{F}$ arises basically as a consequence of the varying amplitude of the linear power spectrum. The amplitude $\sigma_{8}(z)$ varies by $4 / 3.2=1.25$ from $z=3$ to $z=2.2$ (neglecting the influence of the cosmological constant at these high redshifts), so the redshift evolution behaves in the same way as the variation with $\sigma_{8}$ in figure 18: $P_{F} / P_{L}$ decreases nearly as fast as $\sigma_{8}(z)^{-2}$ at low $k$ and $\mu$, with an increase of $\beta$ with $\sigma_{8}(z)$, and the value $k_{n a}$ where the quadrupole changes sign decreases (i.e., shifts to larger scales) with $\sigma_{8}(z)$. It is interesting to note that this value of $k_{n a}$ is not shifting at all under the large change of $\bar{F}$ from 0.851 in the left panel to 0.696 in the right panel, and depends on the redshift only despite the clear variations of the shape of the curves with $\bar{F}$.

The bias and redshift distortion factors are shown in figure 25 for the fiducial model, for three different cases. The red squares show their true evolution when $\bar{F}(z)$ is varied at the same time as the redshift. The blue circles show the isolated redshift evolution, when keeping $\bar{F}=0.781$ fixed to its value at $z=2.6$, and the green triangles show the result when varying only $\bar{F}$ but keeping fixed $z=2.6$ (only the redshift $z$ is shown in the horizontal axis but the green points are all for $z=2.6$, and for $\bar{F}$ equal to the value at the redshift in the axis).

The redshift evolution of $b_{\tau \delta}$ for fixed $\bar{F}$ is, as mentioned in $\S 6.1$, equivalent to the variation with the power spectrum amplitude, increasing with redshift nearly as $b_{\tau \delta} \propto \sigma_{8}(z)^{-1}$, in agreement with the blue points in the middle panel. When varying $\bar{F}$ at fixed redshift, $P_{F}$ increases rapidly as $\bar{F}$ declines but this is mostly due to the relation between $b_{F \delta}$ and $b_{\tau \delta}$. The residual variation left for the optical depth bias on $\bar{F}$, shown as the green points, 

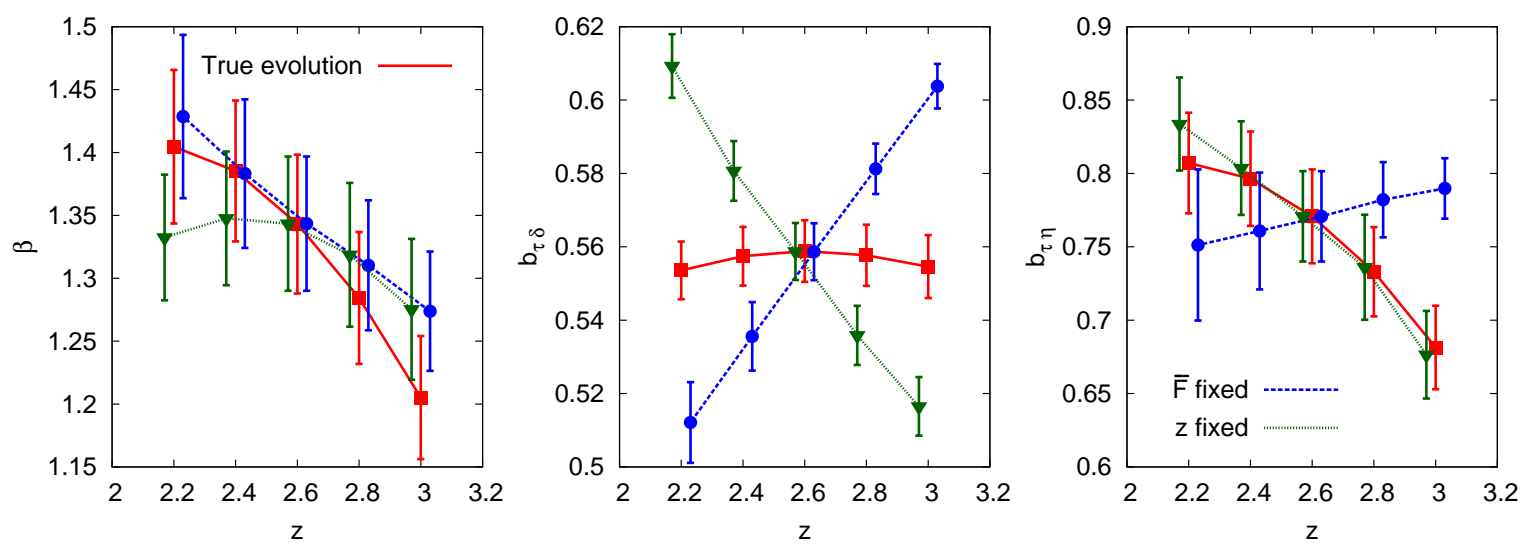

Figure 25. Bias and redshift distortion factors for the fiducial model. Red squares show our standard result for the redshift evolution, when $\bar{F}(z)$ varies with redshift as observed. Blue circles show the redshift evolution when we fix $\bar{F}=0.781$, equal to the value at $z=2.6$. Green triangles show the variations at a fixed redshift $z=2.6$, varying only $\bar{F}(z)$ to the value it has at each of our standard redshift outputs. Blue circles (green triangles) are shifted slightly right (left) to avoid superposition of errorbars.

is actually of opposite sign: as $\bar{F}$ decreases, regions of declining overdensity in the IGM are responsible for the dominant variations measured in the Ly $\alpha$ forest spectra, and they have a decreasing physical bias factor. The true evolution we can measure of $b_{\tau \delta}$, shown as the red points, is nearly constant with redshift due to a fortuitous cancellation of the effects of declining power spectrum amplitude and declining $\bar{F}$ with redshift. This cancellation is, however, not a very precise prediction from our simulations because it is sensitive to the fit we use to obtain the bias factors, as can be seen from the redshift evolution of the fiducial model bias factors for the free $q_{2}$ fit that is shown in figure 14 .

The right panel of figure 25 shows that the usual decrease of $b_{\tau \eta}$ with redshift is due to the variation of $\bar{F}$. In fact, for fixed $\bar{F}, b_{\tau \eta}$ actually has a slow increase with redshift. The reason why the redshift distortion factor $\beta$ generally decreases with redshift is therefore not very straightforward. At fixed $\bar{F}$, the evolution of $\beta$ has a relatively simple explanation: the decreasing power spectrum amplitude with redshift implies an increase of $b_{\tau \delta}$ with redshift, while $b_{\tau \eta}$ does not change much at fixed $\bar{F}$, so $\beta$ decreases with redshift. However, the reason why the ratio $b_{\tau \eta} / b_{\tau \delta}$ has a redshift evolution that is not strongly changed by the variation of $\bar{F}$ is not so simple.

The non-linear parameters of the fits can be seen in figure 30. Some of the variations with redshift correspond to the variations we have seen before with the amplitude in figure 23: the parameters $q_{1}, k_{v}^{a_{v}}$ and $a_{v}$ increase with redshift for fixed $\bar{F}$. However, these parameters also increase with $\bar{F}$ and the result for the true evolution is a much slower variation. It is interesting that the parameter $k_{p}$ also varies with redshift when computed at fixed $\bar{F}$ and that this is also cancelled by the dependence on $\bar{F}$, an effect that likely depends on the slope of the density-temperature relation.

\subsection{Temperature-Density relation}

To finish this section, we investigate the dependence of $P_{F} / P_{L}$ on the temperature-density relation. First, we compare in figure 27 two models that have both $\gamma=1$ (a flatter relation than in our fiducial model, which has $\gamma=1.6$ ), when we vary the temperature $T_{0}$ at the 

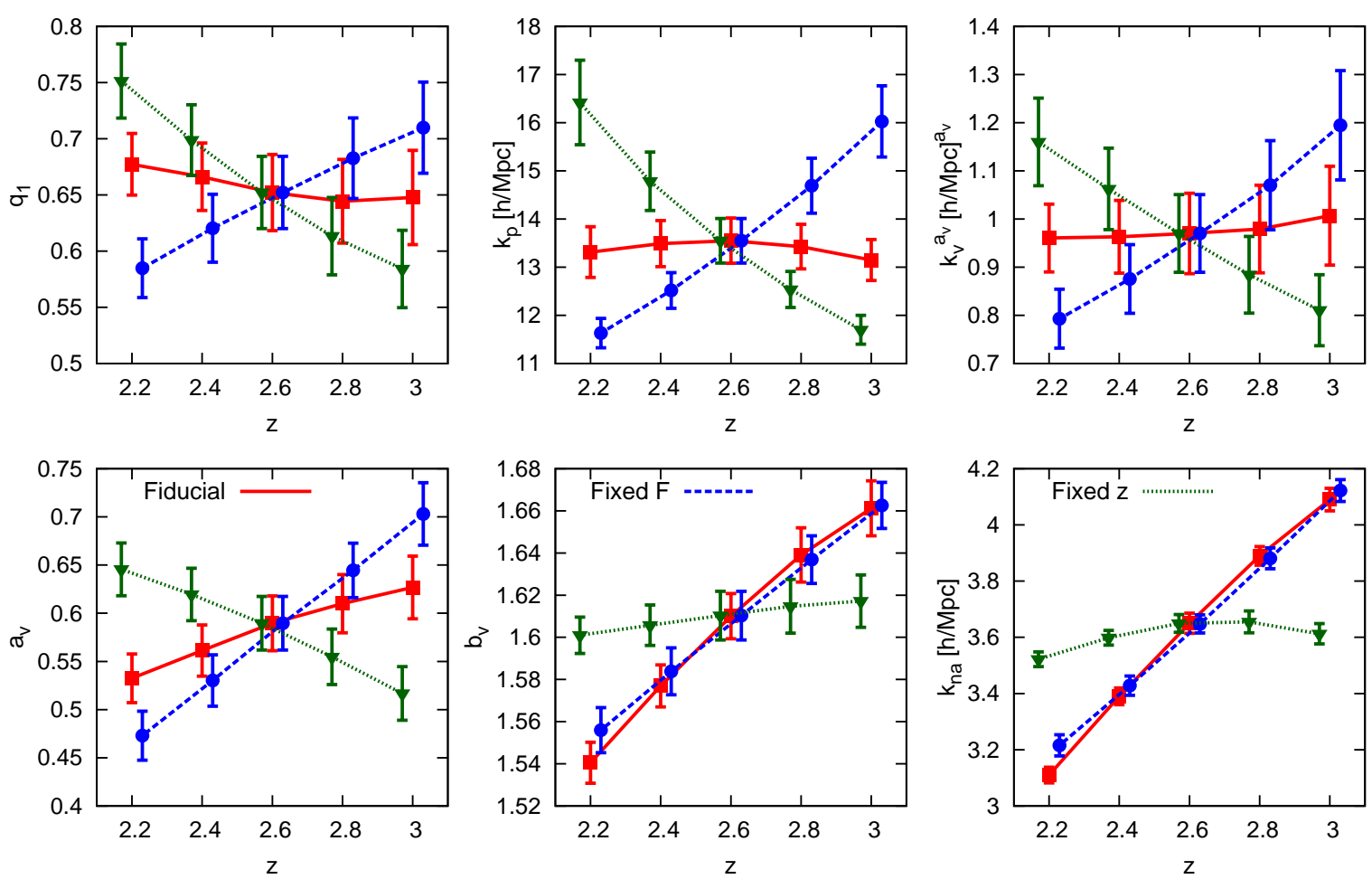

Figure 26. Parameters of the non-linear function $D_{1}(k, \mu)$ for the cases of true redshift evolution (red squares), fixing $\bar{F}$ to its value at $z=2.6$ (blue circles), and fixing $z=2.6$ but using the value of $\bar{F}$ corresponding to each redshift (green triangles).

mean density from $10^{4} \mathrm{~K}$ in model G1T4, shown as colored points and curves, to $10^{4.3} \mathrm{~K}$ in model G1.0, shown as black curves and points. The results are shown at $z=2.6$ and the fits are for $q_{2}=0$. A lower temperature reduces the Jeans length and, as expected, the damping of the power at small scales is reduced, as seen clearly in figure 27. This change in the smallscale power induces a modification of the bias factors on large scales, which as discussed for previous cases, depends on the requirement of keeping a fixed value of $\bar{F}$. Unfortunately, when we compare the difference of the colored and black points at low $k$ in figure 27 with the difference between the fits, we see that whereas the points do not indicate any variation of $\beta$ with temperature (the difference in the points is constant at low $k$ without an appreciable dependence on $\mu$ ), the fits show a different behavior: they reflect the difference between the two models at low $\mu$, but at high $\mu$ the two curves are nearly the same, implying a higher $\beta$ for the low-temperature models. This results from the need to obtain a good fit to the well-determined points at high $k$, and the large errors at low $k$, and therefore the implied small change of $\beta$ with temperature is not reliable.

The more reliable modification induced by the gas temperature is the variation of the scale $k_{n a}$ : a lower gas temperature implies lower velocity dispersions and therefore smaller non-linear effects on the power spectrum anisotropy, so the scale of sign reversal of the power spectrum quadrupole decreases. Figure 27 shows that $k_{n a}$ decreases by $\sim 10 \%$ when the temperature increases by a factor of two. This provides a possible clue to resolve degeneracies with $\sigma_{8}$ and $\bar{F}$ in using the shape of the non-linear power to constrain the IGM temperature from future measurements (e.g., by using both the value of $k_{n a}$ and the damping rate of the 


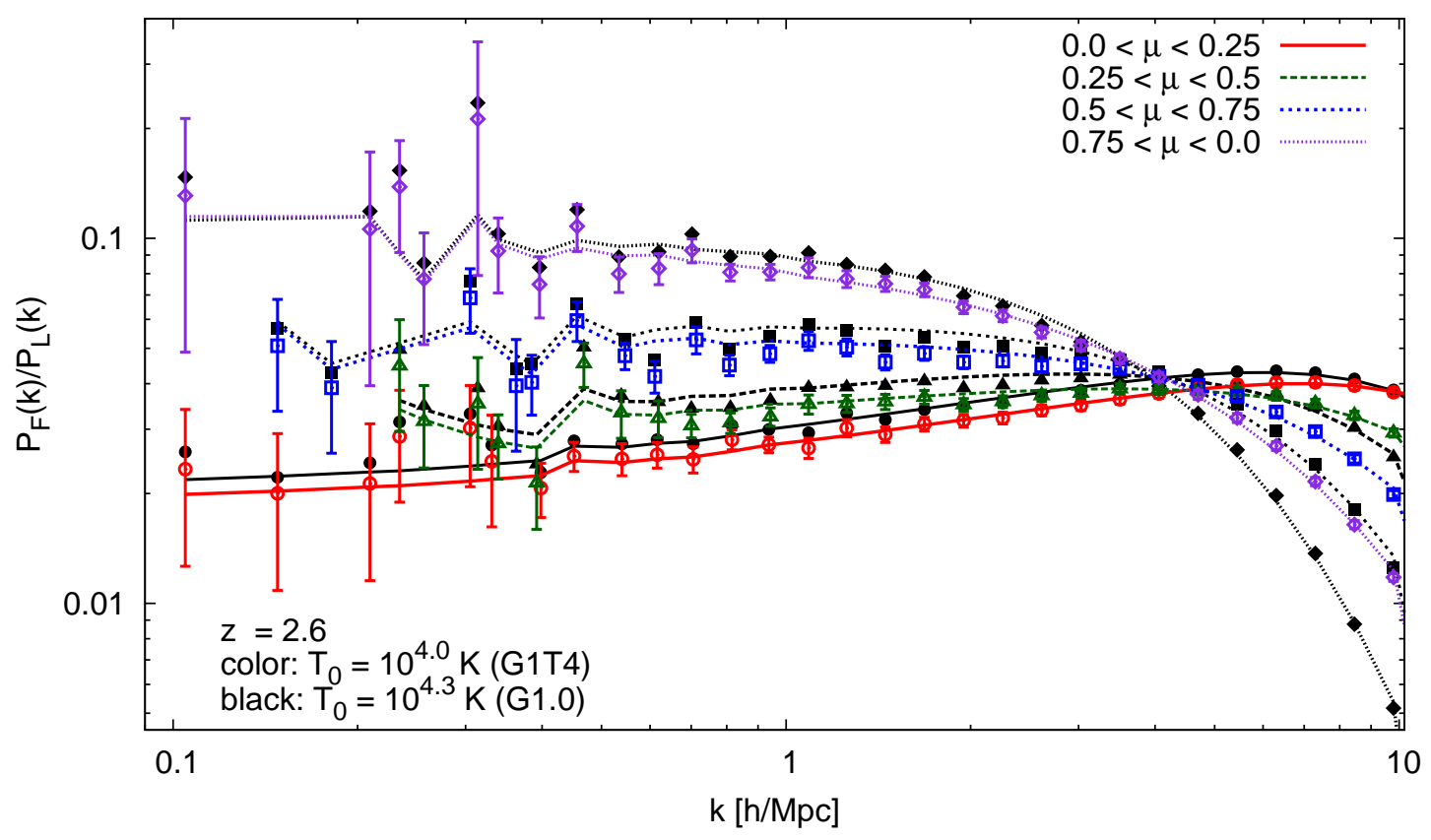

Figure 27. Dependence of $P_{F} / P_{L}$ on the gas temperature, at $z=2.6$. The two models shown have a temperature-density relation slope $\gamma=1$, and the gas temperature is twice higher for the black points compared to the colored ones.

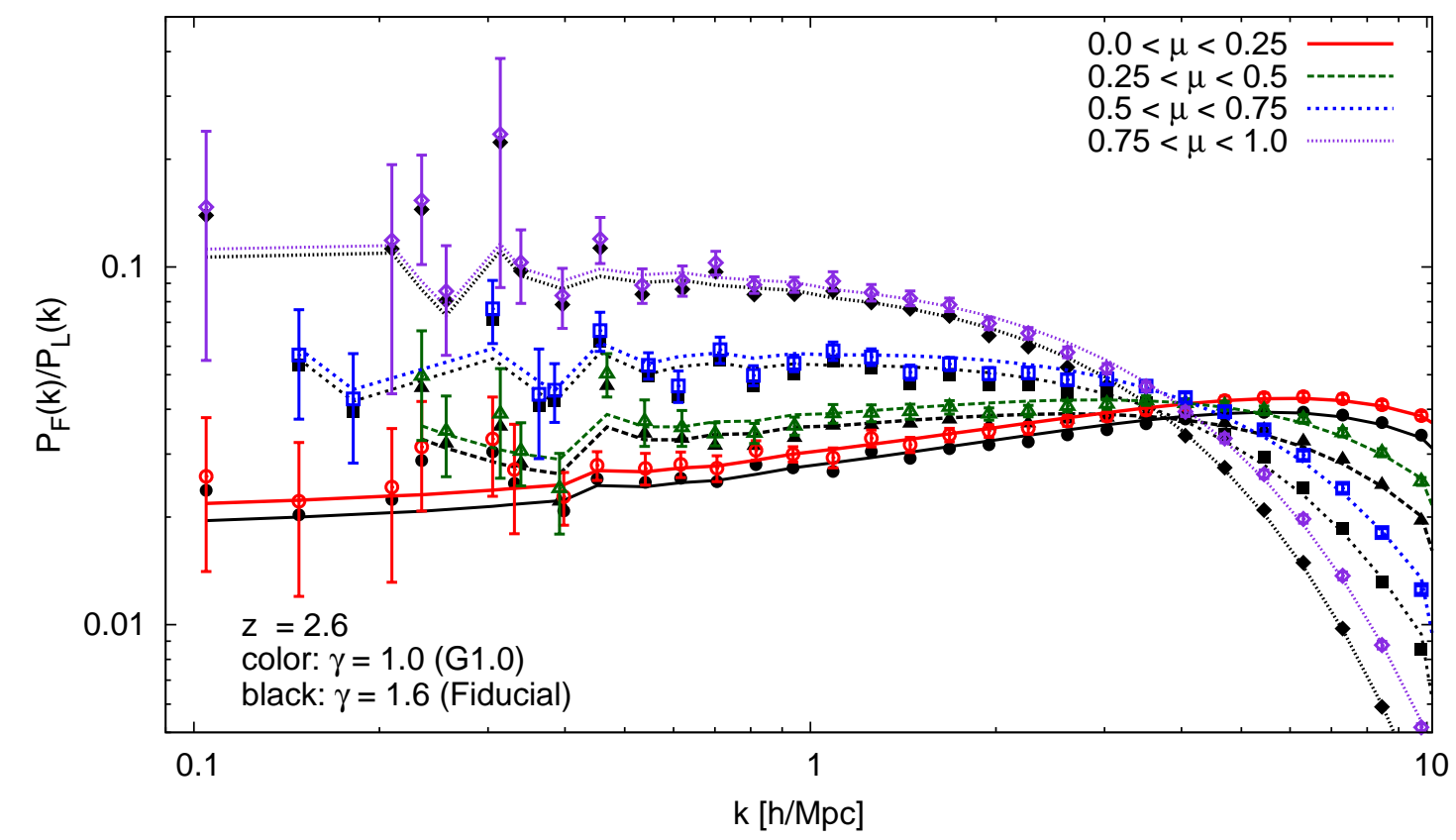

Figure 28. Dependence of $P_{F} / P_{L}$ on the slope of the temperature-density relation at $z=2.6$. The black points are for the fiducial model, and the colored points show the G1.0 model result, when modifying the slope to $\gamma=1$ (which is the same model as the black curves in figure 27 . 
power at higher $k$ ).

The effect of varying the temperature-density relation slope is shown in figure 28 , where we compare the G1.0 model (with $\gamma=1$; colored curves and points) with the fiducial model ( $\gamma=1.6$; black curves and points), also at $z=2.6$. The temperature is kept fixed at the mean density, at $T_{0}=10^{4.3} \mathrm{~K}$. There is also a reduction of the damping at small scales in the G1.0 model, probably because the relevant value of the temperature is at a density above the mean when we use $\bar{F}(z=2.6)=0.781$, where the G1.0 model has the lower temperature. This agrees also with the fact that the value of $k_{n a}$ decreases with the slope $\gamma$, in the same way as it decreases with the temperature as in figure 27. However, the reduction in slope produces an increase of the bias factors, contrary to the temperature reduction which decreases them. Therefore, sufficiently careful observations of the non-linear shape of the power spectrum may help disentangle many of the properties of the IGM.
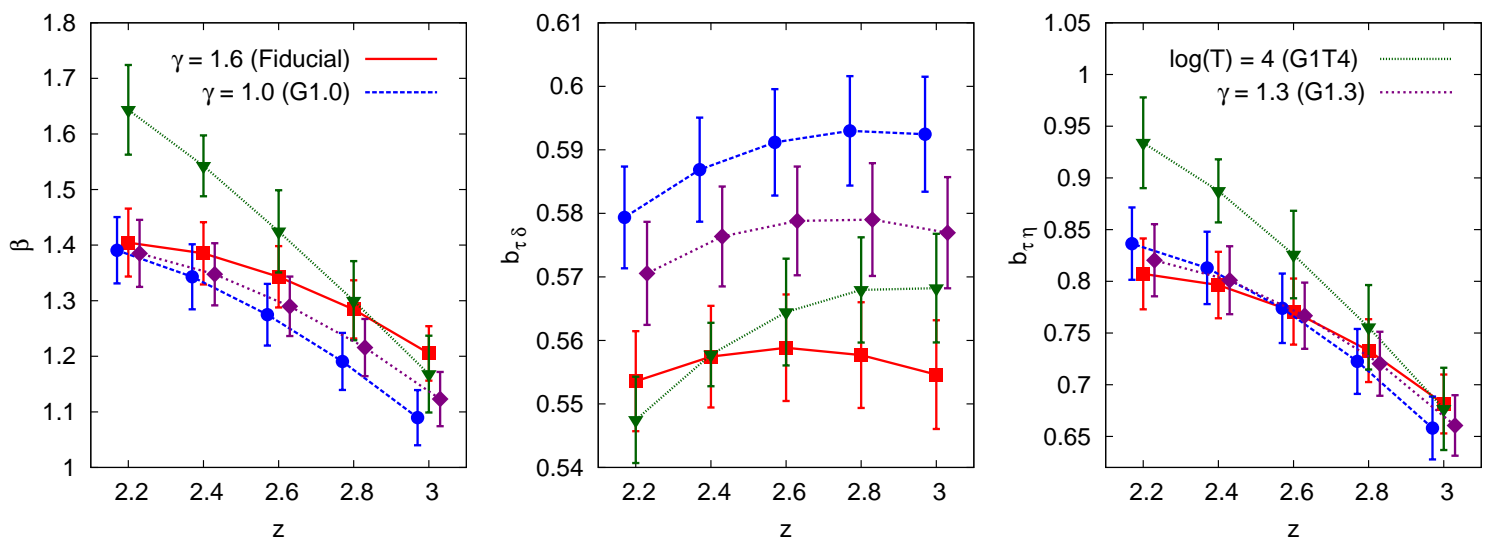

Figure 29. Bias and redshift distortion factors for models with varying temperature-density relation, $T=T_{0}(\rho / \bar{\rho})^{\gamma-1}$. Three models have a temperature at the mean density $T_{0}=10^{4.3} \mathrm{~K}$, and slopes $\gamma=1.6$ (fiducial, red squares), $\gamma=1.3$ (G1.3, purple rhombi), and $\gamma=1$ (G1.0, blue circles). The model G1T4 (green triangles) also has $\gamma=1$ and a lower temperature, $T_{0}=10^{4} \mathrm{~K}$.

The redshift distortion and bias factors of these models are shown in figure 29. A fourth model of intermediate slope between the fiducial and G1.0 models is added. The figure confirms the general property we have mentioned above: the density bias factor increases with the temperature $T_{0}$ and decreases with the slope $\gamma$, but the variation is small. The general conclusion that $\beta$ drops with redshift is valid for all models, but the steeper redshift evolution of $\beta$ for the low-temperature model G1T4 seen in the left panel is not so reliable because of the difference between the fits and the actual simulation points at low $k$ described in figure 27. This detailed question can only be resolved by examining more simulations of different temperature-density relations on large boxes.

The non-linear parameters obtained from the same fits are shown in figure 30. The parameter $k_{p}$ increases as expected when $T_{0}$ is reduced for the G1T4 model (green triangles), corresponding to a decreasing Jeans scale, and the flattening of the slope also increases $k_{p}$ even for the models of fixed $T_{0}$, as mentioned above. However, this variation of $k_{p}$ with $T_{0}$ has a large dependence on redshift. A much clearer effect of reducing $T_{0}$ is the reduction of $k_{v}^{a_{v}}$ and $a_{v}$, which is not affected by the slope $\gamma$. The model dependence of $k_{n a}$, which has much smaller errors due to the much smaller degeneracy with other parameters, shows clearly the effects that we have referred to earlier: the wavenumber $k_{n a}$ decreases with the 

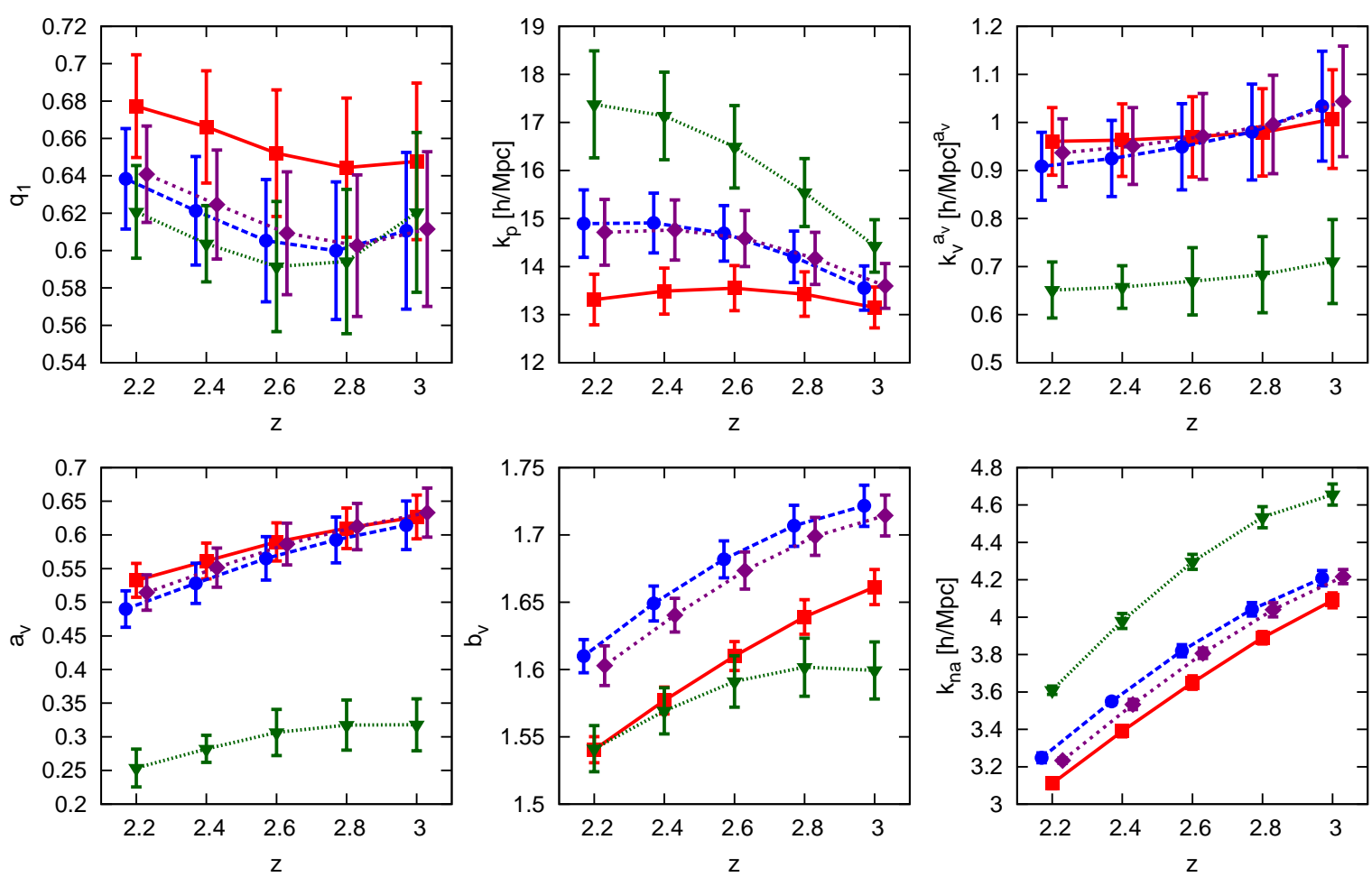

Figure 30. Parameters of the non-linear function $D_{1}(k, \mu)$ for the fits to the same four models with different temperature-density relation as in figure 29 .

temperature $T_{0}$, and decreases also to a smaller extent when the slope $\gamma$ is increased. There are also interesting changes of the power-law index $b_{v}$ for the $\mu$-dependence with the slope $\gamma$, suggesting again that a precise measurement of the multiple characteristics of the nonlinear power spectrum in future observational studies can provide a precise diagnostic of the evolution of the IGM.

\section{Discussion}

The study of the Ly $\alpha$ forest has reached an age of maturity. The observations are providing precision measurements of the transmission correlation function or power spectrum: the one-dimensional power spectrum is measured to very high accuracy [46], and the threedimensional correlation function has been measured on large-scales with a new method that treats the distortion introduced by quasar continuum fitting [3]. In this context, reliable theoretical predictions are needed to confront the large amount of observational results that are becoming available from large surveys [34].

In this work, we have introduced a new formula for fitting the numerical results on the Ly $\alpha$ power spectrum from simulations of structure formation, designated as $D_{1}$ in equation (3.6). This formula was obtained from a guess for how the non-linear correction should depend on the fluctuation amplitude in Fourier space, $\Delta^{2}(k)$, but it is not derived from any perturbation theory. It is based on trial-and-error experimentation to see which formula best fits the numerical results, and on the previous work by M03, where a $\mu$-dependence of the form $D \propto \exp \left[-A(k) \mu^{b_{v}}\right]$ was proposed. We have found in this work that in fact, this 
$\mu$-dependence of the non-linear correction is a surprisingly good fit to the simulation results (figure 6), but we have no analytic explanation for this. In the future, it would be desirable to attempt developing a second-order perturbation theory for modeling the non-linear coupling of a given Fourier mode to all other modes, which suggests that $D(k, \mu)$ should be given by an integral over all possible wavevectors $\mathbf{k}^{\prime}$ of the amplitude product $\Delta\left(\mathbf{k}^{\prime}\right) \Delta\left(\mathbf{k}-\mathbf{k}^{\prime}\right)$ times a coupling strength [37], instead of our simple term proportional to $\Delta^{2}(k)$.

The form of the non-linear power spectrum is clearly predicted by our simulations, and basically agrees with that found in M03. We have characterized it in terms of the wavenumber, $k_{n a}$, at which non-linear anisotropy due to velocity dispersion and thermal broadening starts dominating over the linear anisotropy of large-scale velocity flows, and the curves of the ratio $P_{F}(k, \mu) / P_{L}(k)$ at different $\mu$ cross each other. At higher wavenumbers the power falls fast for high $\mu$ due to thermal broadening, and more slowly at low $\mu$ due to the Jeans scale of gas at the densities of typical absorption systems, with a characteristic smoothing scale given by our $k_{p}$ parameter. As the precision and reliability of the hydrodynamic simulations improves, more accurate predictions on the precise shape of the non-linear power spectrum should be possible. This requires only analyzing enough simulations with large boxes and sufficient resolution to reduce the systematic effects we discuss in $\S 5$. The details of the shape of $P_{F}(k, \mu)$ should probably allow to measure independently the amplitude and slope of the power spectrum $P_{L}(k)$ and the temperature-density relation, as described in $\S 6$.

The numerical results also make a prediction of the two bias factors of the Ly $\alpha$ forest. The form of the linear power spectrum is that in equation (2.5), but the values of the two bias factors depend on all the model parameters that affect the non-linear scales. We have introduced the bias factors based on the effective optical depth in equation (2.4), which are physically interpretable in the same way as the bias of galaxy populations. Our predictions for these bias factors are not highly accurate because of the sampling variance of our simulations with limited box size and, to a smaller extent, the limited resolution. Moreover, their fitted values depend on the formula that is used to fit the whole shape of the non-linear power spectrum, as illustrated by the different results we obtain when the $q_{2}$ parameter is left free or is set to zero. An improvement over the work presented here should be to consider also the cross-power spectrum of $\delta_{F}$ with the initial conditions of the simulation, which can give an improved estimate of the bias factors. Nevertheless, as explained in $\S 5$ we believe that our estimates of the bias factors are reliable within $\sim 10 \%$. The basic result is that the density bias factor is $\sim 0.6$ for the standard CDM $\Lambda$ model, decreases with the power spectrum amplitude as $\sigma_{8}^{-1}$, and is close to constant with redshift as a result of the opposite effects of the decreasing power spectrum amplitude and decreasing $\bar{F}$ with redshift. The peculiar velocity gradient bias, which is unity for an isotropic tracer, is predicted to be close to but below unity, and decreasing with redshift. The resulting redshift distortion factor, $\beta$, is $\sim 1.4$ at $z=2.2$, decreases with redshift and increases with the amplitude $\sigma_{8}$.

The difference of $b_{\tau \eta}$ from unity is an important prediction of the hydrodynamic simulations we are studying. If the absorption line features in the Ly $\alpha$ forest were arising from a series of discrete clouds that are small compared to the scales where cosmological structures are entering the non-linear regime, then a change in the large-scale peculiar velocity gradient $\eta$ (see equation 2.2) should simply compress or stretch the fixed absorption profiles into a smaller or larger redshift range, depending on the sign of the change in $\eta$. Even though the lines increase their blending when $\eta$ increases, the effective optical depth still varies as $(1-\eta)^{-1}$, and so $b_{\tau \eta}$ must be exactly unity.

It is only when the absorbers have internal velocity flows that are systematically aligned 
with the large-scale peculiar velocity gradients that they can respond differently to a varying $\eta$ at fixed $\delta$. This is the situation when the absorbers in the Ly $\alpha$ forest correspond to collapsing structures in the cosmic web of the IGM: if $\eta$ increases, the absorption lines in the forest are compressed into a narrower redshift range, but they also become internally narrower and therefore more saturated owing to their own internal velocity gradients, which are correlated with those on large-scales. As a consequence, the effective optical depth they produce increases slower than $\eta$, and $b_{\tau \eta}$ is less than one. This difference from unity should be more pronounced when the absorbers are low-density structures following the velocity gradients on large-scales, and less pronounced when the dominant absorbing structures are due to high-density gas in more virialized regions, where the velocity gradients and dispersions are randomized and less correlated with the surrounding large-scale structure. As $\bar{F}$ increases closer to unity (i.e., there is less mean absorption), the dominant absorption lines correspond to more overdense gas, and $b_{\tau \eta}$ should be closer to one. This agrees with the behavior we find in our simulations (see green points in right panel of figure 25). We also find the value of $b_{\tau \eta}$ is substantially larger than $b_{\tau \Gamma}$ in equation (2.8), as shown in the right panel of figure 4, which would be the value predicted if the internal peculiar velocities of the absorbers could be modeled linearly [51].

We now compare our results on the non-linear power spectrum with the ones obtained by M03. The general shape of $P_{F} / P_{L}$ is in very good agreement, as can be seen by comparing Figure 9 in M03 with several of our figures, for example, figure 18. For a more quantitative comparison, it is useful to compute first the power spectrum normalization at $z=2.25$, the only redshift at which results were shown in M03. Our Planck model, which has a very similar spectral index and gas temperature as the M03 model, has $\sigma_{8}(z=0)=0.834$ and $\Omega_{m 0}=0.3175$, implying $\sigma_{8}(z=2.25)=0.321$, and $\sigma_{8}(z=2.6)=0.291$. The model used in M03 had $\sigma_{8}(z=0)=0.79$ and $\Omega_{m 0}=0.4$, implying $\sigma_{8}(z=2.25)=0.290$, very close to the amplitude of our fiducial model at $z=2.6$. Figure 9 of M03 also shows the feature that the curves at different $\mu$ cross each other nearly at the same point, at $k_{n a} \simeq 4 h / \mathrm{Mpc}$. This is in fact nearly the same value found for our Planck model at $z=2.6$, as seen in figure 24, in agreement with our finding that this scale depends mostly on the power spectrum amplitude (there is also a dependence on gas temperature, which is similar in our Planck model and the one in M03). The way the power drops at $k>k_{n a}$ for the two models is also fairly similar.

At low $k$, the value found for the redshift distortion parameter in M03 of $\beta=1.58$ is higher than ours. Taking into account the lower power spectrum normalization and lower value of $\bar{F}(z=2.25)=0.8$ used in M03 compared to our fiducial model, our prediction for the model used in M03 would be $\beta \simeq 1.32$ when using our $q_{2}=0$ fits, as derived by interpolating results in our figures 20 and 25 . Using our free $q_{2}$ fits generally reduces the predicted value of $\beta$ by a further few percent. There is therefore a substantial discrepancy in the redshift distortion factor. The density bias factor given in Table 1 of M03 of $b_{F \delta}^{2}=0.0173$ is, on the other hand, in very good agreement with our $q_{2}=0$ fits: the implied $b_{\tau \delta}=0.59$ for the mean transmission $\bar{F}=0.8$ used in M03 is nearly equal to our predicted value for the Planck model (see figure 20), which has the same amplitude at $z=2.6$ and a similar value of $\bar{F}$ at this redshift. This suggests that the discrepancy in $\beta$ is related to a higher prediction for $b_{\tau \eta}$ from M03 compared to our models. However, if we use our free $q_{2}$ fits, then our value of $b_{\tau \delta}$ increases by $\sim 12 \%$, and then the discrepancy in $\beta$ is mostly due to the low prediction for $b_{\tau \delta}$ from M03.

The reason for this discrepancy and the correct prediction for $\beta$ and $b_{\tau \delta}$ will only be resolved with the analysis of more simulations on large boxes. Understanding the systematic 
errors and the accuracy of the prediction of $b_{\tau \eta}$ from hydrodynamic simulations is especially important because if this value can be predicted to an accuracy better than $1 \%$, then a precise measurement of $\beta$ and $b_{\tau \delta}$ yields an observational determination of the growth factor logarithmic derivative, $f(\Omega)$, from equation (2.6), and therefore a fundamental test of the presence and evolution of dark energy in the Universe at high redshift.

We also find that the variations of the bias factors with the model parameters specified in Table 1 of M03 are in broad agreement with our results described in $\S 6$.

\subsection{Comparison to observations}

There are several observations of the Ly $\alpha$ forest power spectrum that our predictions can be compared to. First, on the linear regime, the measurements of the Ly $\alpha$ autocorrelation on large scales yielded a first detection of redshift distortions in [52] using BOSS. More recently, an improved method that effectively corrects for distortions introduced by continuum fitting has been presented in [3], allowing for accurate measurements of the bias factors. Apart from this, the one-dimensional power spectrum is a projection of the full redshift space transmission power spectrum which includes non-linear effects, and was measured first in [41], and then in [46] with the higher accuracy allowed by BOSS. The full redshift-space power spectrum down to small scales, where non-linear effects are important, can also be measured from the BOSS data and from close pairs of quasars where correlations can be measured down to the smallest scales. Results have been presented constraining the Jeans scale [33], but a complete analysis of the available data in terms of the models for the full shape of the three-dimensional power spectrum is yet to be done.

Here, we make only a brief comparison with the measured bias factors in [3], leaving the comparison with the one-dimensional results and other measurements of the non-linear regime for future papers. The result of [3], obtained at a mean redshift $z=2.3$ from a fit restricted to large scales (from $40 h^{-1} \mathrm{Mpc}$ to $160 h^{-1} \mathrm{Mpc}$ ) is $\beta=1.39 \pm 0.11$, and $b_{F \delta}(1+\beta)=-0.374 \pm 0.007$; the second quantity is chosen because it has the smallest marginalized observational error.

The measured value of $\beta$ is in excellent agreement with our predictions. Our Planck model is the one that uses parameters consistent with present constraints from the Cosmic Background Radiation, and predicts $\beta=1.4$ at $z=2.3$ for the $q_{2}=0$ fit, which drops to 1.3 for free $q_{2}$. We note from figures 12 and 14, however, that this prediction is subject to a substantial systematic error due to box size and resolution effects. This value also has some sensitivity to other poorly determined parameters of the IGM, in particular to $\bar{F}$, but we see in figure 25 (green triangles) that we expect a weak variation of $\beta$ in the range $0.85<\bar{F}<0.78$.

Comparing the predicted and measured values of $b_{\tau \delta}$ is more model-dependent. The results of [3] are obtained using their fiducial model, with $\Omega_{m 0}=0.27$ and $\sigma_{8}(z=0)=0.79$, implying $\sigma_{8}(z=2.3)=0.307$. While the measurement of $\beta$ is not affected by this fiducial model used to fit the data, the density bias factor is affected because the Ly $\alpha$ transmission power that is actually observed is proportional to $\left[\sigma_{8}(z) b_{F \delta}\right]^{2}$. Our Planck model has a slightly higher amplitude, $\sigma_{8}(z=2.3)=0.313$. Correcting for this, if our Planck model had been used for the data analysis, the [3] result would then be $b_{F \delta}(1+\beta)=-0.367 \pm 0.007$. The theoretical prediction of our Planck model at $z=2.3$ using our $q_{2}=0$ fit, and our standard value of $\bar{F}(z=2.3)=0.836$, is $b_{\tau \delta}=0.586$ and $\beta=1.40$, implying $b_{F \delta}=-0.105$, and $b_{F \delta}(1+\beta)=-0.253$. This is substantially smaller than the measured value. 
There are two systematic errors that dominate the uncertainty in this comparison. The first is the error of our theoretical predictions, dominated by the uncertainty in fitting the bias parameters from the low- $k$ modes. If we use the fit that includes the $q_{2}$ free parameter in equation (3.6), the predicted density bias factor increases to $b_{\tau \delta}=0.68$ (see Table 4 in Appendix B), and with $\beta=1.3, b_{F \delta}(1+\beta)=-0.28$. This is still substantially below the observed value. The second systematic error is related to the value of $\bar{F}$, which is poorly determined by observations. Assuming that $\bar{F}=0.8$ at $z=2.3$ (instead of the value $\bar{F}=0.836$ derived from equation 3.1), we find from the results in figure 25 that the predicted bias for $q_{2}=0$ would change to $b_{\tau \delta} \simeq 0.56$, implying $b_{F \delta}=-0.125$ and $b_{F \delta}(1+\beta)=-0.300$. If we combine both systematic errors (assuming the higher $b_{\tau \delta}$ from our free $q_{2}$ fits and a low mean transmission $\bar{F}=0.8$ at $z=2.3$ ) yields a value $b_{F \delta}(1+\beta)=-0.334$, closer but still not consistent with the observational determination of [3].

It remains to be seen if the mean transmission from the Ly $\alpha$ forest is in fact substantially lower than was found by [31], or if there is a large discrepancy between the predicted value of $b_{\tau \delta}$ from the simulations analyzed in this paper and the observations. The recent determinations of $\bar{F}$ by [1] give a value similar to that of [31]. It is also possible that the value of the bias factors is altered by the presence of intensity fluctuations in the ionizing background. The need to understand the observed values of the bias factors highlights the importance of obtaining more precise predictions from cosmological simulations.

\section{Conclusions}

The analysis of a variety of simulations of the $\mathrm{Ly} \alpha$ forest we have presented highlights the fact that the detailed form of the Ly $\alpha$ transmission power spectrum in redshift space is in principle predictable from a well-defined theory of the IGM. The theory assumes that photoionization by the cosmic ultraviolet background and the gravitational evolution of structure that arises from primordial fluctuations in the Cold Dark Matter scenario determine the properties of the IGM and the Ly $\alpha$ power spectrum. Detailed observations in the future should provide tests of the validity of this simple theory, and investigate to what extent the effects of galactic winds, quasar jets, fluctuations of the ionizing background intensity, and inhomogeneities of the density-temperature relation arising from reionization, have an impact on the observable power spectrum and other characteristics of the Ly $\alpha$ forest.

Much work remains to be done both on the theoretical and data analysis front to fully confront the model predictions with observations. The existing data from BOSS and close quasar pairs that have been individually observed should provide powerful measurements of the full Ly $\alpha$ power spectrum, beyond the constraints obtained from the one-dimensional analysis [46, 47]. This will also be complemented by new surveys of absorption spectra that will improve on the BOSS results. At the same time, the theory needs to be further developed with the analysis of more simulations on large boxes, as they are made possible by modern computational technology. An improved understanding of the effects of variations on the physical model of the IGM is necessary before one can use the non-linear Ly $\alpha$ power spectrum to constrain fundamental parameters in cosmology related, for example, to neutrino masses or other modifications of the dark matter sector.

To make the results of our simulations accessible for comparing to future simulations or observations, we are providing in Appendix B the fit parameters to the most useful models that are presented in this paper. At the same time, for more direct comparisons to the results of our simulations, we are also providing the results for $P_{F}(k, \mu)$ of all our models, with the 
bins that we have used to obtain all our fits. These data are publicly available at GibHub ${ }^{3}$ repositories (https://github.com/andreuandreu/3D_Power_spectrum_modes_tables).

\section{Acknowledgments}

We would like to thank Michael Blomqvist, David Kirkby, José Oñorbe and Nathalie PalanqueDelabrouille for discussions. AA and JM are supported in part by Spanish grant AYA201233938. MV is supported by the ERC Starting Grant "cosmoIGM", PRIN INAF and PRIN MIUR. I would like to thank the universe for existing and letting me study it.

\footnotetext{
${ }^{3}$ https://github.com/andreuandreu/3D_Power_spectrum_modes_tables
} 


\section{References}

[1] Becker, G. D., Hewett, P. C., Worseck, G., \& Prochaska, J. X. 2013, Mon. Not. Roy. Astron. Soc. , 430, 2067

[2] Bhanot, G. 1988, Reports on Progress in Physics, 51, 429

[3] Blomqvist, M., Kirkby, D., Bautista, J. E., et al. 2015, ArXiv e-prints, arXiv:1504.06656

[4] Boera, E., Murphy, M. T., Becker, G. D., \& Bolton, J. S. 2014, Mon. Not. Roy. Astron. Soc. , 441, 1916

[5] Bolton, J. S., Becker, G. D., Haehnelt, M. G., \& Viel, M. 2014, Mon. Not. Roy. Astron. Soc. , 438,2499

[6] Bolton, J. S., Viel, M., Kim, T.-S., Haehnelt, M. G., \& Carswell, R. F. 2008, Mon. Not. Roy. Astron. Soc. , 386, 1131

[7] Borde, A., Palanque-Delabrouille, N., Rossi, G., et al. 2014, JCAP , 7, 5

[8] Busca, N. G., Delubac, T., Rich, J., et al. 2013, A\&A, 552, A96

[9] Cen, R. 1992, Astrophys. J. Sup. , 78, 341

[10] —. 2002, Astrophys. J. Sup. , 141, 211

[11] —. 2010, Formation and Evolution of Galaxies and the Intergalactic Medium, nASA Proposal \#10-ATP10-73

[12] Cen, R., Miralda-Escudé, J., Ostriker, J. P., \& Rauch, M. 1994, Astrophys. J. Let. , 437, L9

[13] Cen, R., \& Ostriker, J. P. 1993, Astrophys. J. , 417, 404

[14] Cen, R. Y., Ostriker, J. P., Jameson, A., \& Liu, F. 1990, Astrophys. J. Let. , 362, L41

[15] Compostella, M., Cantalupo, S., \& Porciani, C. 2013, Mon. Not. Roy. Astron. Soc. , 435, 3169

[16] Croft, R. A. C., Weinberg, D. H., Bolte, M., et al. 2002, Astrophys. J. , 581, 20

[17] Croft, R. A. C., Weinberg, D. H., Katz, N., \& Hernquist, L. 1998, Astrophys. J. , 495, 44

[18] Dawson, K. S., Schlegel, D. J., Ahn, C. P., et al. 2013, AJ, 145, 10

[19] Delubac, T., Bautista, J. E., Busca, N. G., et al. 2014, ArXiv e-prints, arXiv:1404.1801

[20] Eisenstein, D. J., Weinberg, D. H., Agol, E., et al. 2011, AJ, 142, 72

[21] Font-Ribera, A., \& Miralda-Escudé, J. 2012, JCAP , 7, 28

[22] Font-Ribera, A., Kirkby, D., Busca, N., et al. 2014, JCAP , 5, 27

[23] Gnedin, N. Y., \& Hui, L. 1998, Mon. Not. Roy. Astron. Soc. , 296, 44

[24] Gontcho A Gontcho, S., Miralda-Escudé, J., \& Busca, N. G. 2014, Mon. Not. Roy. Astron. Soc. , 442, 187

[25] Hamilton, T. 1992, in Bulletin of the American Astronomical Society, Vol. 24, American Astronomical Society Meeting Abstracts, 1248

[26] Hasselfield, M., Hilton, M., Marriage, T. A., et al. 2013, JCAP , 7, 8

[27] Hernquist, L., Katz, N., Weinberg, D. H., \& Miralda-Escudé, J. 1996, Astrophys. J. Let. , 457, L51

[28] Hui, L., \& Gnedin, N. Y. 1997, Mon. Not. Roy. Astron. Soc. , 292, 27

[29] Kaiser, N. 1987, Mon. Not. Roy. Astron. Soc. , 227, 1

[30] Katz, N., Weinberg, D. H., \& Hernquist, L. 1996, Astrophys. J. Sup. , 105, 19

[31] Kim, T.-S., Bolton, J. S., Viel, M., Haehnelt, M. G., \& Carswell, R. F. 2007, Mon. Not. Roy. 
Astron. Soc. , 382, 1657

[32] Kollmeier, J. A., Miralda-Escudé, J., Cen, R., \& Ostriker, J. P. 2006, Astrophys. J. , 638, 52

[33] Kulkarni, G., Hennawi, J. F., Oñorbe, J., Rorai, A., \& Springel, V. 2015, ArXiv e-prints, arXiv:1504.00366

[34] Lee, K.-G., Bailey, S., Bartsch, L. E., et al. 2013, AJ, 145, 69

[35] Lukić, Z., Stark, C. W., Nugent, P., et al. 2015, Mon. Not. Roy. Astron. Soc. , 446, 3697

[36] McDonald, P. 2003, Astrophys. J. , 585, 34

[37] —. 2006, Phys. Rev. D , 74, 103512

[38] McDonald, P., \& Eisenstein, D. J. 2007, Phys. Rev. D , 76, 063009

[39] McDonald, P., Miralda-Escudé, J., Rauch, M., et al. 2000, Astrophys. J. , 543, 1

[40] McDonald, P., Seljak, U., Cen, R., Bode, P., \& Ostriker, J. P. 2005, Mon. Not. Roy. Astron. Soc. , 360, 1471

[41] McDonald, P., Seljak, U., Burles, S., et al. 2006, Astrophys. J. Sup. , 163, 80

[42] McQuinn, M., Lidz, A., Zaldarriaga, M., et al. 2009, Astrophys. J. , 694, 842

[43] Meiksin, A. A. 2009, Reviews of Modern Physics, 81, 1405

[44] Miralda-Escudé, J., Cen, R., Ostriker, J. P., \& Rauch, M. 1996, Astrophys. J., 471, 582

[45] Miralda-Escudé, J., \& Rees, M. J. 1994, Mon. Not. Roy. Astron. Soc. , 266, 343

[46] Palanque-Delabrouille, N., Yèche, C., Borde, A., et al. 2013, A\&A, 559, A85

[47] Palanque-Delabrouille, N., Yèche, C., Lesgourgues, J., et al. 2015, JCAP , 2, 45

[48] Pontzen, A. 2014, Phys. Rev. D , 89, 083010

[49] Rauch, M. 1998, ARAA, 36, 267

[50] Regan, J. A., Haehnelt, M. G., \& Viel, M. 2007, Mon. Not. Roy. Astron. Soc. , 374, 196

[51] Seljak, U. 2012, JCAP , 3, 4

[52] Slosar, A., Font-Ribera, A., Pieri, M. M., et al. 2011, JCAP , 9, 1

[53] Slosar, A., Iršič, V., Kirkby, D., et al. 2013, JCAP , 4, 26

[54] Springel, V. 2005, Mon. Not. Roy. Astron. Soc. , 364, 1105

[55] Theuns, T., Leonard, A., Efstathiou, G., Pearce, F. R., \& Thomas, P. A. 1998, Mon. Not. Roy. Astron. Soc. , 301, 478

[56] Theuns, T., Viel, M., Kay, S., et al. 2002, Astrophys. J. Let. , 578, L5

[57] Viel, M., Becker, G. D., Bolton, J. S., \& Haehnelt, M. G. 2013, Phys. Rev. D , 88, 043502

[58] Viel, M., Haehnelt, M. G., \& Springel, V. 2004, Mon. Not. Roy. Astron. Soc. , 354, 684

[59] Viel, M., Schaye, J., \& Booth, C. M. 2013, Mon. Not. Roy. Astron. Soc. , 429, 1734

[60] Zhang, Y., Anninos, P., \& Norman, M. L. 1995, in Bulletin of the American Astronomical Society, Vol. 27, American Astronomical Society Meeting Abstracts, 1412 


\section{A Dependence of the Bias Factors on the Minimum Error parameter}

The fits to the non-linear Ly $\alpha$ power spectrum we have obtained depend on several technical parameters for binning the power spectrum and evaluating the $\chi^{2}$ function, which are described in $\S 3.3$. We have checked that our results for the best fits are not strongly dependent on the way the Fourier modes are binned. As long as our parameter $k_{t}$ is not too small, the rebinning of Fourier modes should not modify the fit. However, the $\epsilon$ parameter that is inserted in the expression for the error in equation 3.2 inevitably affects our fits. The introduction of this parameter is necessary to reduce the weight of the high- $k$ modes for our fits, because the number of modes below $k$ increases as $k^{3}$. We note that if the total number of bins $n_{b}$ at $k>k_{t}$ to evaluate the power $P_{F}$ is modified (in our fits this was fixed to $n_{b}=256$ ), then our fits are modified depending on the paremeter $\epsilon n_{b}^{-3 / 2}$, because the fits depend on the effective weight assigned to modes in each fixed region of Fourier space.

The choice of the parameter $\epsilon$ cannot be made in a very objective way. As $\epsilon$ is increased, an increasing discrepancy in the high- $k$ modes is allowed, taking into account that our fitting formula is not a perfect theory that should precisely match the results obtained from numerical simulations, which have very small statistical errors at high $k$ that are at some point smaller than the systematic errors. Reducing the weight of the high- $k$ modes allows for a better fit of the bias factors from the low- $k$ modes.
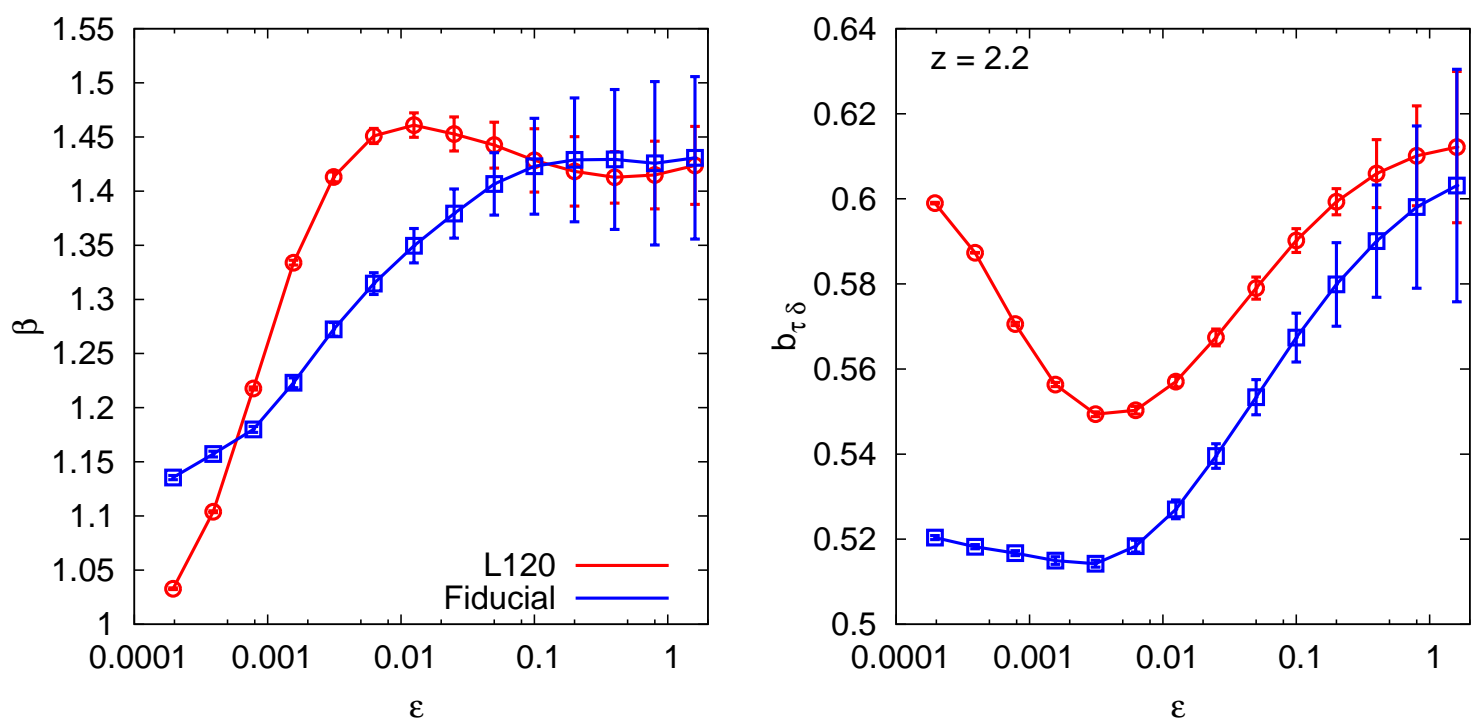

Figure 31. Dependence of the redshift distortion factor $\beta$ and the density bias factor $b_{\tau \delta}$ on the parameter $\epsilon$ determining a minimum error in our fit with the $D_{1}$ formula with $q_{2}=0$. Blue squares (red circles) show the result for our fiducial (L120) model, at $z=2.2$.

Therefore, we look into the variation of the redshift distortion factor and the density bias factor in the two panels of figure 31. As $\epsilon$ is increased, the value of $\beta$ converges just near the value we have chosen in this paper $\epsilon=0.05$. The value of $b_{\tau \delta}$ continues to increase above this value of $\epsilon$, by $\sim 10 \%$ as $\epsilon$ is increased to 1 . Interestingly, the bias factor reaches a value $b_{\tau \delta} \simeq 0.61$ at very high $\epsilon$, very close to the result we obtain in our fits with free $q_{2}$ for these models (see figure 14). This suggests that our models with free $q_{2}$ yield more accurate values of the bias factors, that are less affected by the need to correctly fit the numerous data 
points for $P_{F}$ at high $k$ from our simulations. Nevertheless, this will need to be tested with results from more simulations on large boxes than are analyzed in this paper. 


\section{B Tables}

Table 2. Bias parameters for simulations with different resolution and box size, for the $q_{2}=0$ fit.

\begin{tabular}{|c|c|c|c|}
\hline$z$ & $\beta$ & $b_{\tau \delta}$ & $b_{\tau \eta}$ \\
\hline \multicolumn{4}{|c|}{$\overline{\text { Fiducial }}$} \\
\hline 3.0 & $1.205 \pm 0.049$ & $.5546 \pm 0.0086$ & $0.681 \pm 0.028$ \\
\hline 2.8 & $1.284=$ & $0.5577 \pm$ & 0.73 \\
\hline 2.6 & $1.343 \pm 0.055$ & $0.5588 \pm 0.0084$ & $0.771 \pm 0$. \\
\hline 2.4 & $1.385 \pm 0.056$ & $0.5574 \pm 0.0080$ & $0.796 \pm 0.032$ \\
\hline 2.2 & $1.405 \pm 0.061$ & $0.5536 \pm 0.0079$ & $0.807 \pm 0.034$ \\
\hline \multicolumn{4}{|c|}{ P1024 } \\
\hline 3.0 & $1.205 \pm 0.050$ & $0.5566 \pm 0.0979$ & $0.684 \pm 0.119$ \\
\hline 2.8 & $1.283 \pm 0.052$ & $0.5597 \pm$ & $0.735 \pm 0.030$ \\
\hline 2.6 & $1.340 \pm 0.056$ & $0.5611 \pm$ & $0.772 \pm 0.033$ \\
\hline 2.4 & $1.381 \pm 0.059$ & $0.5599 \pm$ & $0.797 \pm 0.034$ \\
\hline 2.2 & $1.403 \pm 0.061$ & 05556 & \\
\hline \multicolumn{4}{|c|}{ L120 } \\
\hline 3.0 & $1.195 \pm$ & $\overline{5710 \pm}$ & $0.695=$ \\
\hline 2.8 & $1.283 \pm 0.040$ & $0.5768 \pm$ & $0.757 \pm 0.024$ \\
\hline 2.6 & $1.355 \pm$ & 0.58 & 0.808 \\
\hline 2.4 & 1.411 & $0.5820=$ & 0.847 \\
\hline 2.2 & $1.443 \pm($ & $0.5789 \pm$ & $0.867 \exists$ \\
\hline \multicolumn{4}{|c|}{$\frac{1}{R 384 C}$} \\
\hline 3.0 & 1.210 & & $\overline{0.6}$ \\
\hline 2.8 & $1.290 \pm$ & 0.5714 & $0.754=$ \\
\hline 2.6 & $1.360 \pm 0.060$ & $0.5732 \pm$ & $0.800 \pm 0.035$ \\
\hline 2.4 & $1.420 \pm 0$ & $0.5715 \pm($ & $0.837 \pm 0.029$ \\
\hline 2.2 & $1.455 \pm 0.064$ & $0.5588 \pm 0.0085$ & $0.844 \exists$ \\
\hline \multicolumn{4}{|c|}{$\frac{1}{R 384}$} \\
\hline 3.0 & $1.180 \pm 0.060$ & $\overline{0.5708 \pm}$ & $0.687 \pm 0.036$ \\
\hline 2.8 & $1.270 \pm 0.060$ & $0.5743 \pm 0.0096$ & $0.746 \pm 0.036$ \\
\hline 2.6 & $1.330 \pm$ & $0.5760 \pm$ & $0.787 \pm 0.035$ \\
\hline 2.4 & $1.380 \pm$ & $0.5758 \pm$ & $0.820 \pm 0.041$ \\
\hline 2.2 & $1.420 \pm 0$ & $0.5703 \pm$ & $0.841 \pm 0.041$ \\
\hline \multicolumn{4}{|c|}{ R640 } \\
\hline 3.0 & 1.25 & & $\overline{0.6}$ \\
\hline 2.8 & $1.316 \pm$ & $0.5456 \pm$ & $0.735 \pm$ \\
\hline 2.6 & $1.364 \pm 0.054$ & $0.5482 \pm 0.0082$ & $0.768 \pm 0.031$ \\
\hline 2.4 & $1.397 \pm 0.056$ & $0.5487 \pm 0.0079$ & $0.791 \pm 0.032$ \\
\hline 2.2 & $1.421 \pm 0.061$ & $0.5461 \pm 0.0085$ & $0.805 \pm 0.034$ \\
\hline
\end{tabular}


Table 3. Non-linear fit parameters for the same simulations as in Table 2.

\begin{tabular}{|c|c|c|c|c|c|c|}
\hline Z & $q_{1}$ & $k_{p}[h / \mathrm{Mpc}]$ & $k_{v}^{a_{v}}[h / \mathrm{Mpc}]^{a_{v}}$ & $a_{v}$ & $b_{v}$ & $k_{n a}$ \\
\hline \multicolumn{7}{|c|}{ Fiducial } \\
\hline 3.0 & $0.648 \pm 0.042$ & $13.1 \pm 0.4$ & $1.007 \pm 0.103$ & $0.627 \pm 0.033$ & $1.66 \pm 0.01$ & $4.09 \pm 0.04$ \\
\hline 2.8 & $0.644 \pm 0.037$ & $13.4 \pm 0.5$ & $0.979 \pm 0.091$ & $0.610 \pm 0.030$ & $1.64 \pm 0.01$ & $3.89 \pm 0.03$ \\
\hline 2.6 & $0.652 \pm 0.034$ & $13.6 \pm 0.5$ & $0.970 \pm 0.084$ & $0.590 \pm 0.028$ & $1.61 \pm 0.01$ & $3.65 \pm 0.04$ \\
\hline 2.4 & $0.666 \pm 0.030$ & $13.5 \pm 0.5$ & $0.963 \pm 0.076$ & $0.561 \pm 0.027$ & $1.58 \pm 0.01$ & $3.39 \pm 0.03$ \\
\hline 2.2 & $0.677 \pm 0.027$ & $13.3 \pm 0.5$ & $0.961 \pm 0.070$ & $0.533 \pm 0.025$ & $1.54 \pm 0.01$ & $3.11 \pm 0.03$ \\
\hline \multicolumn{7}{|c|}{$\mathrm{P} 1024$} \\
\hline 3.0 & $0.644 \pm 0.042$ & $13.2 \pm 0.4$ & $0.997 \pm 0.100$ & $0.634 \pm 0.032$ & $1.66 \pm 0.02$ & $4.09 \pm 0.04$ \\
\hline 2.8 & $0.642 \pm 0.037$ & $13.5 \pm 0.6$ & $0.973 \pm 0.091$ & $0.617 \pm 0.030$ & $1.63 \pm 0.02$ & $3.89 \pm 0.03$ \\
\hline 2.6 & $0.650 \pm 0.035$ & $13.7 \pm 0.5$ & $0.964 \pm 0.084$ & $0.597 \pm 0.029$ & $1.60 \pm 0.02$ & $3.65 \pm 0.04$ \\
\hline 2.4 & $0.665 \pm 0.031$ & $13.6 \pm 0.5$ & $0.959 \pm 0.075$ & $0.567 \pm 0.026$ & $1.57 \pm 0.02$ & $3.39 \pm 0.03$ \\
\hline 2.2 & $0.679 \pm 0.028$ & $13.4 \pm 0.5$ & $0.956 \pm 0.069$ & $0.537 \pm 0.025$ & $1.53 \pm 0.01$ & $3.11 \pm 0.03$ \\
\hline \multicolumn{7}{|c|}{ L120 } \\
\hline 3.0 & $0.661 \pm 0.032$ & $11.3 \pm 0.2$ & $0.803 \pm 0.064$ & $0.460 \pm 0.026$ & $1.55 \pm 0.01$ & $4.01 \pm 0.03$ \\
\hline 2.8 & $0.635 \pm 0.028$ & $12.0 \pm 0.3$ & $0.782 \pm 0.057$ & $0.471 \pm 0.024$ & $1.56 \pm 0.01$ & $3.80 \pm 0.03$ \\
\hline 2.6 & $0.626 \pm 0.026$ & $12.7 \pm 0.4$ & $0.772 \pm 0.053$ & $0.469 \pm 0.023$ & $1.56 \pm 0.01$ & $3.56 \pm 0.02$ \\
\hline 2.4 & $0.633 \pm 0.023$ & $13.2 \pm 0.4$ & $0.771 \pm 0.048$ & $0.456 \pm 0.021$ & $1.54 \pm 0.01$ & $3.29 \pm 0.02$ \\
\hline 2.2 & $0.643 \pm 0.020$ & $13.5 \pm 0.6$ & $0.778 \pm 0.046$ & $0.439 \pm 0.020$ & $1.52 \pm 0.01$ & $3.00 \pm 0.02$ \\
\hline \multicolumn{7}{|c|}{ R384C } \\
\hline 3.0 & $0.592 \pm 0.051$ & $14.0 \pm 0.6$ & $0.786 \pm 0.104$ & $0.504 \pm 0.042$ & $1.57 \pm 0.01$ & $4.15 \pm 0.05$ \\
\hline 2.8 & $0.578 \pm 0.045$ & $14.9 \pm 0.8$ & $0.774 \pm 0.095$ & $0.509 \pm 0.038$ & $1.58 \pm 0.01$ & $3.92 \pm 0.05$ \\
\hline 2.6 & $0.584 \pm 0.039$ & $15.5 \pm 0.9$ & $0.773 \pm 0.083$ & $0.500 \pm 0.036$ & $1.58 \pm 0.02$ & $3.67 \pm 0.04$ \\
\hline 2.4 & $0.602 \pm 0.032$ & $15.9 \pm 1.1$ & $0.778 \pm 0.064$ & $0.478 \pm 0.028$ & $1.55 \pm 0.01$ & $3.40 \pm 0.03$ \\
\hline 2.2 & $0.644 \pm 0.027$ & $15.4 \pm 0.8$ & $0.822 \pm 0.060$ & $0.458 \pm 0.024$ & $1.52 \pm 0.02$ & $3.12 \pm 0.03$ \\
\hline \multicolumn{7}{|c|}{ R384 } \\
\hline 3.0 & $0.586 \pm 0.051$ & $12.9 \pm 0.5$ & $0.921 \pm 0.120$ & $0.617 \pm 0.041$ & $1.66 \pm 0.02$ & $4.13 \pm 0.05$ \\
\hline 2.8 & $0.584 \pm 0.043$ & $13.3 \pm 0.5$ & $0.894 \pm 0.104$ & $0.600 \pm 0.038$ & $1.64 \pm 0.02$ & $3.93 \pm 0.05$ \\
\hline 2.6 & $0.594 \pm 0.041$ & $13.6 \pm 0.6$ & $0.885 \pm 0.097$ & $0.578 \pm 0.037$ & $1.61 \pm 0.01$ & $3.68 \pm 0.04$ \\
\hline 2.4 & $0.612 \pm 0.038$ & $13.7 \pm 0.7$ & $0.877 \pm 0.089$ & $0.547 \pm 0.034$ & $1.58 \pm 0.01$ & $3.42 \pm 0.03$ \\
\hline 2.2 & $0.634 \pm 0.030$ & $13.5 \pm 0.6$ & $0.880 \pm 0.079$ & $0.514 \pm 0.032$ & $1.54 \pm 0.01$ & $3.13 \pm 0.03$ \\
\hline \multicolumn{7}{|c|}{ R640 } \\
\hline 3.0 & $0.642 \pm 0.042$ & $14.9 \pm 0.8$ & $1.023 \pm 0.111$ & $0.651 \pm 0.034$ & $1.71 \pm 0.03$ & $4.14 \pm 0.04$ \\
\hline 2.8 & $0.633 \pm 0.038$ & $15.1 \pm 0.7$ & $0.998 \pm 0.094$ & $0.636 \pm 0.030$ & $1.68 \pm 0.02$ & $3.95 \pm 0.04$ \\
\hline 2.6 & $0.636 \pm 0.034$ & $15.1 \pm 0.7$ & $0.984 \pm 0.085$ & $0.614 \pm 0.028$ & $1.65 \pm 0.02$ & $3.71 \pm 0.04$ \\
\hline 2.4 & $0.645 \pm 0.030$ & $14.8 \pm 0.6$ & $0.969 \pm 0.077$ & $0.582 \pm 0.027$ & $1.61 \pm 0.01$ & $3.45 \pm 0.02$ \\
\hline 2.2 & $0.656 \pm 0.027$ & $14.4 \pm 0.7$ & $0.950 \pm 0.071$ & $0.543 \pm 0.026$ & $1.57 \pm 0.02$ & $3.16 \pm 0.03$ \\
\hline
\end{tabular}


Table 4. Bias parameters for $D_{1}$ with $q_{2}$ set free.

\begin{tabular}{|c|c|c|c|}
\hline 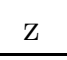 & $\beta$ & $b_{\tau \delta}$ & $b_{\tau \eta}$ \\
\hline \multicolumn{4}{|c|}{ Fiducial } \\
\hline 3.0 & $1.179 \pm 0.061$ & $.0010+0.0120$ & $0.128-0.00$ \\
\hline 2.8 & $1.256 \pm$ & 6096 & \\
\hline 2.6 & $1.292 \pm 0$ & $6206 \pm 0$ & $823 \pm$ \\
\hline 2.4 & $1.321 \pm 0$ & $36+0$ & $856+0.044$ \\
\hline 2.2 & $1.347 \pm 0.0$ & $06206+0$ & $.882 \pm 0.031$ \\
\hline \multicolumn{4}{|c|}{$\mathrm{R} 384 \mathrm{C}$} \\
\hline 3.0 & $1.200 \pm 0$ & $0.5981 \pm 0$. & $0.732 \pm 0.045$ \\
\hline 2.8 & $1.288 \pm 0$ & & $0.797 \pm 0.044$ \\
\hline 2.6 & 1.3 & 0.6 & $0.848 \pm$ \\
\hline 2.4 & $1.399 \pm 0.078$ & $0.6147 \pm 0$. & $0.887 \pm 0.050$ \\
\hline 2.2 & $1.426 \pm$ & & 047 \\
\hline \multicolumn{4}{|c|}{ L120 } \\
\hline 3.0 & $1.204=$ & 98 & $0.713 \pm 0.021$ \\
\hline 2.8 & $1.296 \pm 0$ & $0.5962 \pm 0$. & $0.791 \pm 0.021$ \\
\hline 2.6 & $1.365 \pm 0$ & $2 \pm 0$ & 036 \\
\hline 2.4 & $1.409 \pm 0$ & $0.6202 \pm 0$ & $0.901 \pm 0.034$ \\
\hline 2.2 & $1.431 \pm 0$ & $0.6249 \pm 0$ & $0.928 \pm 0.026$ \\
\hline \multicolumn{4}{|c|}{$\mathrm{L} 80$} \\
\hline 3.0 & 1.12 & & $\overline{032}$ \\
\hline 2.8 & $1.210 \pm($ & 0.6 & $0.774 \pm 0.023$ \\
\hline 2.6 & $1.280 \pm 0$ & $0.6344 \pm 0$ & $0.834 \pm 0.024$ \\
\hline 2.4 & $1.340 \pm 0$ & $0.6375 \pm 0.0078$ & $0.881 \pm 0.021$ \\
\hline 2.2 & $1.350 \pm 0.030$ & $0.6474 \pm 0.0120$ & $0.907 \pm 0.024$ \\
\hline \multicolumn{4}{|c|}{ Planck } \\
\hline 3.0 & $1.072 \pm 0.059$ & $0.6462 \pm 0$. & $0.657 \pm 0.045$ \\
\hline 2.8 & $1.155 \pm 0$ & $0.6573 \pm 0$. & $0.723 \pm 0.049$ \\
\hline 2.6 & $1.254 \pm$ & $0.6601 \pm 0$. & $0.791 \pm 0.034$ \\
\hline 2.4 & $1.290 \pm 0.074$ & $0.6784 \pm 0.0154$ & $0.840 \pm 0.056$ \\
\hline 2.2 & $1.316 \pm 0.057$ & $0.6751 \pm 0.0141$ & $0.858 \pm 0.044$ \\
\hline
\end{tabular}


Table 5. Non-linear fit parameters for $D_{1}$ with $q_{2}$ set free.

\begin{tabular}{|c|c|c|c|c|c|c|}
\hline $\mathrm{Z}$ & $q_{1}$ & $q_{2}$ & $k_{p}[h / M p c]$ & $k_{v}^{a_{v}}[h / M p c]^{a_{v}}$ & $a_{v}$ & $b_{v}$ \\
\hline \multicolumn{7}{|c|}{$\overline{\text { Fiducial }}$} \\
\hline 3.0 & $0.104 \pm 0.121$ & $0.444 \pm 0.093$ & $10.1 \pm 0.5$ & $0.516 \pm 0.114$ & $0.248 \pm 0.072$ & $1.66 \pm 0.02$ \\
\hline 2.8 & $0.086 \pm 0.110$ & $0.417 \pm 0.078$ & $9.9 \pm 0.5$ & $0.493 \pm 0.087$ & $0.217 \pm 0.069$ & $1.63 \pm 0.02$ \\
\hline 2.6 & $0.068 \pm 0.101$ & $0.390 \pm 0.063$ & $9.6 \pm 0.5$ & $0.483 \pm 0.052$ & $0.190 \pm 0.056$ & $1.61 \pm 0.02$ \\
\hline 2.4 & $0.057 \pm 0.085$ & $0.368 \pm 0.049$ & $9.2 \pm 0.3$ & $0.480 \pm 0.028$ & $0.156 \pm 0.045$ & $1.57 \pm 0.02$ \\
\hline 2.2 & $0.090 \pm 0.052$ & $0.316 \pm 0.027$ & $8.9 \pm 0.3$ & $0.493 \pm 0.016$ & $0.145 \pm 0.031$ & $1.54 \pm 0.01$ \\
\hline \multicolumn{7}{|c|}{ R384C } \\
\hline 3.0 & $0.207 \pm 0.208$ & $0.320 \pm 0.159$ & $11.2 \pm 1.0$ & $0.468 \pm 0.165$ & $0.208 \pm 0.120$ & $1.57 \pm 0.01$ \\
\hline 2.8 & $0.202 \pm 0.132$ & $0.289 \pm 0.094$ & $11.4 \pm 0.8$ & $0.462 \pm 0.111$ & $0.207 \pm 0.093$ & $1.57 \pm 0.01$ \\
\hline 2.6 & $0.203 \pm 0.073$ & $0.267 \pm 0.041$ & $11.2 \pm 0.4$ & $0.467 \pm 0.035$ & $0.198 \pm 0.042$ & $1.57 \pm 0.01$ \\
\hline 2.4 & $0.208 \pm 0.083$ & $0.246 \pm 0.035$ & $11.0 \pm 0.3$ & $0.475 \pm 0.019$ & $0.182 \pm 0.027$ & $1.54 \pm 0.01$ \\
\hline 2.2 & $0.233 \pm 0.062$ & $0.213 \pm 0.023$ & $10.8 \pm 0.2$ & $0.499 \pm 0.015$ & $0.181 \pm 0.019$ & $1.51 \pm 0.01$ \\
\hline \multicolumn{7}{|c|}{ L120 } \\
\hline 3.0 & $0.529 \pm 0.115$ & $0.117 \pm 0.102$ & $10.6 \pm 0.6$ & $0.684 \pm 0.196$ & $0.362 \pm 0.085$ & $1.54 \pm 0.01$ \\
\hline 2.8 & $0.398 \pm 0.112$ & $0.190 \pm 0.087$ & $10.5 \pm 0.6$ & $0.583 \pm 0.167$ & $0.290 \pm 0.076$ & $1.55 \pm 0.01$ \\
\hline 2.6 & $0.316 \pm 0.105$ & $0.226 \pm 0.070$ & $10.3 \pm 0.7$ & $0.529 \pm 0.118$ & $0.233 \pm 0.074$ & $1.54 \pm 0.01$ \\
\hline 2.4 & $0.264 \pm 0.091$ & $0.241 \pm 0.060$ & $9.9 \pm 0.6$ & $0.501 \pm 0.069$ & $0.183 \pm 0.065$ & $1.53 \pm 0.01$ \\
\hline 2.2 & $0.245 \pm 0.086$ & $0.232 \pm 0.051$ & $9.6 \pm 0.6$ & $0.497 \pm 0.046$ & $0.153 \pm 0.059$ & $1.51 \pm 0.01$ \\
\hline \multicolumn{7}{|l|}{$\mathrm{L} 80$} \\
\hline 3.0 & $0.144 \pm 0.232$ & $0.430 \pm 0.202$ & $8.9 \pm 0.8$ & $0.514 \pm 0.286$ & $0.212 \pm 0.158$ & $1.61 \pm 0.01$ \\
\hline 2.8 & $0.130 \pm 0.133$ & $0.394 \pm 0.108$ & $9.0 \pm 0.6$ & $0.490 \pm 0.108$ & $0.190 \pm 0.093$ & $1.59 \pm 0.01$ \\
\hline 2.6 & $0.117 \pm 0.110$ & $0.367 \pm 0.080$ & $8.9 \pm 0.5$ & $0.480 \pm 0.059$ & $0.167 \pm 0.071$ & $1.56 \pm 0.01$ \\
\hline 2.4 & $0.147 \pm 0.082$ & $0.320 \pm 0.054$ & $8.8 \pm 0.4$ & $0.493 \pm 0.041$ & $0.161 \pm 0.054$ & $1.53 \pm 0.01$ \\
\hline 2.2 & $0.120 \pm 0.083$ & $0.308 \pm 0.047$ & $8.5 \pm 0.4$ & $0.484 \pm 0.021$ & $0.123 \pm 0.050$ & $1.50 \pm 0.01$ \\
\hline \multicolumn{7}{|c|}{ Planck } \\
\hline 3.0 & $0.0004 \pm 0.2241$ & $0.787 \pm 0.220$ & $11.8 \pm 1.2$ & $0.552 \pm 0.201$ & $0.178 \pm 0.108$ & $1.64 \pm 0.03$ \\
\hline 2.8 & $-0.0168 \pm 0.1686$ & $0.711 \pm 0.143$ & $12.0 \pm 1.0$ & $0.548 \pm 0.126$ & $0.203 \pm 0.073$ & $1.65 \pm 0.03$ \\
\hline 2.6 & $0.0004 \pm 0.1241$ & $0.651 \pm 0.113$ & $11.8 \pm 1.0$ & $0.545 \pm 0.115$ & $0.195 \pm 0.073$ & $1.64 \pm 0.02$ \\
\hline 2.4 & $-0.0020 \pm 0.1108$ & $0.623 \pm 0.069$ & $10.9 \pm 0.5$ & $0.517 \pm 0.027$ & $0.152 \pm 0.035$ & $1.62 \pm 0.02$ \\
\hline 2.2 & $0.0405 \pm 0.0890$ & $0.534 \pm 0.049$ & $10.6 \pm 0.3$ & $0.534 \pm 0.022$ & $0.139 \pm 0.029$ & $1.60 \pm 0.01$ \\
\hline
\end{tabular}


Table 6. Bias parameters for Eulerian, Lagrangian and Planck simulations, for $q_{2}=0$.

\begin{tabular}{|c|c|c|c|}
\hline $\mathrm{z}$ & $\beta$ & $b_{\tau \delta}$ & $b_{\tau \eta}$ \\
\hline \multicolumn{4}{|c|}{ Fiducial } \\
\hline 3.0 & $1.21 \pm 0.049$ & $0.555 \pm 0.0086$ & $0.681 \pm 0.028$ \\
\hline 2.8 & $1.28 \pm 0.052$ & $0.558 \pm 0.0083$ & $0.733 \pm 0.030$ \\
\hline 2.6 & $1.34 \pm 0.055$ & $0.559 \pm 0.0084$ & $0.771 \pm 0.032$ \\
\hline 2.4 & $1.39 \pm 0.056$ & $0.557 \pm 0.0080$ & $0.796 \pm 0.032$ \\
\hline 2.2 & $1.40 \pm 0.061$ & $0.554 \pm 0.0079$ & $0.807 \pm 0.034$ \\
\hline \multicolumn{4}{|c|}{ Eulerian } \\
\hline 2.9 & $1.29 \pm 0.073$ & $0.557 \pm 0.011$ & $0.737 \pm 0.042$ \\
\hline 2.6 & $1.51 \pm 0.084$ & $0.561 \pm 0.010$ & $0.870 \pm 0.048$ \\
\hline 2.3 & $1.63 \pm 0.083$ & $0.557 \pm 0.011$ & $0.941 \pm 0.047$ \\
\hline \multicolumn{4}{|c|}{ Lagrangian } \\
\hline 3.0 & $1.03 \pm 0.055$ & $0.555 \pm 0.011$ & $0.583 \pm 0.032$ \\
\hline 2.8 & $1.14 \pm 0.059$ & $0.561 \pm 0.011$ & $0.653 \pm 0.034$ \\
\hline 2.6 & $1.24 \pm 0.064$ & $0.563 \pm 0.011$ & $0.720 \pm 0.037$ \\
\hline 2.4 & $1.33 \pm 0.069$ & $0.558 \pm 0.010$ & $0.769 \pm 0.039$ \\
\hline 2.2 & $1.47 \pm 0.070$ & $0.556 \pm 0.0097$ & $0.854 \pm 0.04$ \\
\hline \multicolumn{4}{|c|}{ Planck } \\
\hline 3.0 & $1.12 \pm 0.056$ & $0.588 \pm 0.0096$ & $0.627 \pm 0.036$ \\
\hline 2.8 & $1.22 \pm 0.055$ & $0.592 \pm 0.0094$ & $0.689 \pm 0.036$ \\
\hline 2.6 & $1.30 \pm 0.057$ & $0.591 \pm 0.0093$ & $0.736 \pm 0.037$ \\
\hline 2.4 & $1.39 \pm 0.058$ & $0.591 \pm 0.0095$ & $0.788 \pm 0.038$ \\
\hline 2.2 & $1.42 \pm 0.058$ & $0.581 \pm 0.0092$ & $0.796 \pm 0.038$ \\
\hline
\end{tabular}


Table 7. Non-linear fit parameters for the Eulerian, Lagrangian and Planck simulation, for $q_{2}=0$.

\begin{tabular}{|c|c|c|c|c|c|c|}
\hline $\mathrm{Z}$ & $q_{1}$ & $k_{p}[h / M p c]$ & $k_{v}^{a_{v}}[h / M p c]^{a_{v}}$ & $a_{v}$ & $b_{v}$ & $k_{n a}$ \\
\hline \multicolumn{7}{|c|}{ Fiducial } \\
\hline 3.0 & $0.648 \pm 0.042$ & $13.1 \pm 0.42$ & $1.01 \pm 0.10$ & $0.627 \pm 0.033$ & $1.66 \pm 0.013$ & $4.09 \pm 0.040$ \\
\hline 2.8 & $0.644 \pm 0.037$ & $13.4 \pm 0.46$ & $0.979 \pm 0.091$ & $0.610 \pm 0.030$ & $1.64 \pm 0.013$ & $3.89 \pm 0.033$ \\
\hline 2.6 & $0.652 \pm 0.034$ & $13.6 \pm 0.47$ & $0.970 \pm 0.084$ & $0.590 \pm 0.028$ & $1.61 \pm 0.011$ & $3.65 \pm 0.036$ \\
\hline 2.4 & $0.666 \pm 0.030$ & $13.5 \pm 0.48$ & $0.963 \pm 0.076$ & $0.561 \pm 0.027$ & $1.58 \pm 0.010$ & $3.39 \pm 0.030$ \\
\hline 2.2 & $0.677 \pm 0.027$ & $13.3 \pm 0.53$ & $0.961 \pm 0.07$ & $0.533 \pm 0.025$ & $1.54 \pm 0.0097$ & $3.11 \pm 0.028$ \\
\hline \multicolumn{7}{|c|}{ Eulerian } \\
\hline 2.9 & $0.719 \pm 0.064$ & $16.1 \pm 0.73$ & $0.734 \pm 0.10$ & $0.413 \pm 0.043$ & $1.65 \pm 0.038$ & $5.09 \pm 0.071$ \\
\hline 2.6 & $0.71 \pm 0.054$ & $18.0 \pm 1.2$ & $0.702 \pm 0.085$ & $0.394 \pm 0.037$ & $1.60 \pm 0.031$ & $4.76 \pm 0.064$ \\
\hline 2.3 & $0.73 \pm 0.046$ & $19.2 \pm 1.8$ & $0.711 \pm 0.082$ & $0.370 \pm 0.034$ & $1.55 \pm 0.028$ & $4.22 \pm 0.065$ \\
\hline \multicolumn{7}{|c|}{ Lagrange } \\
\hline 3.0 & $0.847 \pm 0.070$ & $16.4 \pm 0.95$ & $1.39 \pm 0.20$ & $0.664 \pm 0.049$ & $1.63 \pm 0.035$ & $4.87 \pm 0.07$ \\
\hline 2.8 & $0.822 \pm 0.063$ & $17.4 \pm 1.3$ & $1.31 \pm 0.18$ & $0.649 \pm 0.046$ & $1.61 \pm 0.032$ & $4.70 \pm 0.059$ \\
\hline 2.6 & $0.824 \pm 0.056$ & $18.1 \pm 1.4$ & $1.24 \pm 0.15$ & $0.619 \pm 0.043$ & $1.59 \pm 0.028$ & $4.46 \pm 0.054$ \\
\hline 2.4 & $0.836 \pm 0.050$ & $18.1 \pm 1.5$ & $1.16 \pm 0.13$ & $0.574 \pm 0.038$ & $1.56 \pm 0.026$ & $4.16 \pm 0.051$ \\
\hline 2.2 & $0.907 \pm 0.043$ & $15.8 \pm 0.8$ & $0.981 \pm 0.085$ & $0.463 \pm 0.029$ & $1.49 \pm 0.019$ & $3.57 \pm 0.034$ \\
\hline \multicolumn{7}{|c|}{ Planck } \\
\hline 3.0 & $0.792 \pm 0.055$ & $17.1 \pm 1.1$ & $1.16 \pm 0.15$ & $0.578 \pm 0.045$ & $1.63 \pm 0.035$ & $4.79 \pm 0.054$ \\
\hline 2.8 & $0.773 \pm 0.050$ & $19.2 \pm 1.4$ & $1.16 \pm 0.14$ & $0.608 \pm 0.040$ & $1.65 \pm 0.031$ & $4.50 \pm 0.053$ \\
\hline 2.6 & $0.781 \pm 0.044$ & $21.1 \pm 1.8$ & $1.15 \pm 0.12$ & $0.611 \pm 0.035$ & $1.64 \pm 0.027$ & $4.19 \pm 0.051$ \\
\hline 2.4 & $0.851 \pm 0.040$ & $19.5 \pm 1.9$ & $1.06 \pm 0.093$ & $0.548 \pm 0.029$ & $1.61 \pm 0.023$ & $3.63 \pm 0.031$ \\
\hline 2.2 & $0.867 \pm 0.035$ & $19.4 \pm 1.5$ & $1.06 \pm 0.081$ & $0.514 \pm 0.027$ & $1.60 \pm 0.021$ & $3.35 \pm 0.029$ \\
\hline
\end{tabular}


Table 8. Values of the bias parameters for different physical properties and for $q_{2}=0$.

\begin{tabular}{|c|c|c|c|}
\hline $\mathrm{z}$ & $\beta$ & $b_{\tau \delta}$ & $b_{\tau \eta}$ \\
\hline \multicolumn{4}{|c|}{ Fiducial $(\sigma=0.88, \log T=4.3)$} \\
\hline 3.0 & $1.205 \pm 0.049$ & $0.5546 \pm 0.0086$ & $0.681 \pm 0.028$ \\
\hline 2.8 & $1.284 \pm$ & $0.5577 \pm$ & $.733 \pm 0.030$ \\
\hline 6 & $3 \pm 0$ & $0.5588 \pm$ & $0.771 \pm$ \\
\hline 2.4 & $1.385 \pm 0$ & $0.5574 \pm 0$ & $0.796 \pm 0.032$ \\
\hline 2.2 & $1.405 \pm 0.061$ & $0.5536 \pm 0$ & $0.807 \pm 0.034$ \\
\hline \multicolumn{4}{|c|}{ S0.76 $(\sigma=0.76)$} \\
\hline 3.0 & $1.086 \pm 0.042$ & $0.6319 \pm 0.0096$ & $0.700 \pm 0.028$ \\
\hline 2.8 & $1.161 \pm 0$ & $0.6373 \pm 0$ & $0.757 \pm$ \\
\hline 2.6 & $1.219 \pm$ & $0.6409 \pm$ & $0.803 \pm 0.032$ \\
\hline 2.4 & $1.257 \pm 0$ & $0.6428 \pm$ & $0.833 \pm 0.033$ \\
\hline 2.2 & $1.284 \pm 0.050$ & $0.6401 \pm$ & \pm 0.033 \\
\hline \multicolumn{4}{|c|}{ S0.64 $(\sigma=0.64)$} \\
\hline 3.0 & $0.965 \pm 0.033$ & $0.7287=$ & $\overline{0.717 \pm}$ \\
\hline 2.8 & $1.035 \pm 0$ & $0.7366=$ & $0.780 \pm 0.028$ \\
\hline 2.6 & 1. & 0.7429 & 30 \\
\hline 2.4 & 1.1 & $0.747 \zeta$ & 031 \\
\hline 2.2 & $1.163 \pm 0.041$ & $0.7490=$ & $0.904 \pm$ \\
\hline \multicolumn{4}{|c|}{ G1.3 $(\gamma=1.3)$} \\
\hline 3.0 & $1.123 \pm 0.049$ & & .029 \\
\hline 2.8 & $1.216 \pm 0$ & 0.57 & 031 \\
\hline 2.6 & $1.290 \pm$ & $0.5788 \pm$ & $0.767 \pm 0.032$ \\
\hline 2.4 & $1.348 \pm$ & 0.57 & $0.801 \pm 0.033$ \\
\hline 2.2 & $1.385 \pm 0.060$ & $0.5705 \pm 0$ & $0.820 \pm 0.035$ \\
\hline \multicolumn{4}{|c|}{ G1.0 $(\gamma=1.0)$} \\
\hline 3.0 & $1.090 \pm 0.050$ & $0.5925 \pm 0$ & $0.658 \pm 0.030$ \\
\hline 2.8 & $1.191 \pm 0.051$ & $0.5930 \pm 0$ & $0.723 \pm 0.031$ \\
\hline 2.6 & $1.275 \pm 0$ & $0.5912 \pm$ & $0.774 \pm 0.034$ \\
\hline 2.4 & $3 \pm 0$ & $0.5869 \pm$ & $3 \pm 0.035$ \\
\hline 2.2 & $1.391 \pm 0.060$ & $0.5794 \pm 0$ & $0.836 \pm 0.035$ \\
\hline \multicolumn{4}{|c|}{ G1T4 $(\gamma=1.0, \log T=4.0)$} \\
\hline$\overline{3.0}$ & $1.168 \pm 0$ & $0.5682 \pm$ & $0.677 \pm 0.040$ \\
\hline 2.8 & $1.300 \pm 0$ & $0.5679 \pm$ & $0.756 \pm 0.041$ \\
\hline 2.6 & $1.425 \pm 0$ & $0.5645 \pm($ & $0.826 \pm 0.042$ \\
\hline 2.4 & $1.543 \pm 0.055$ & $0.5578 \pm 0.0050$ & $0.887 \pm 0.031$ \\
\hline 2.2 & $1.643 \pm 0.081$ & $0.5475 \pm 0.0068$ & $0.934 \pm 0.044$ \\
\hline
\end{tabular}


Table 9. Values of the fitting parameters for different physical properties and for $q_{2}=0$.

\begin{tabular}{|c|c|c|c|c|c|c|}
\hline Z & $q_{1}$ & $k_{p}[h / M p c]$ & $k_{v}^{a_{v}}[h / M p c]^{a_{v}}$ & $a_{v}$ & $b_{v}$ & $k_{n a}$ \\
\hline \multicolumn{7}{|c|}{ Fiducial $(\sigma=0.88, \log T=4.3)$} \\
\hline 3.0 & $0.648 \pm 0.042$ & $13.1 \pm 0.4$ & $1.007 \pm 0.103$ & $0.627 \pm 0.033$ & $1.66 \pm 0.01$ & $4.09 \pm 0.04$ \\
\hline 2.8 & $0.644 \pm 0.037$ & $13.4 \pm 0.5$ & $0.979 \pm 0.091$ & $0.610 \pm 0.030$ & $1.64 \pm 0.01$ & $3.89 \pm 0.03$ \\
\hline 2.6 & $0.652 \pm 0.034$ & $13.6 \pm 0.5$ & $0.970 \pm 0.084$ & $0.590 \pm 0.028$ & $1.61 \pm 0.01$ & $3.65 \pm 0.04$ \\
\hline 2.4 & $0.666 \pm 0.030$ & $13.5 \pm 0.5$ & $0.963 \pm 0.076$ & $0.561 \pm 0.027$ & $1.58 \pm 0.01$ & $3.39 \pm 0.03$ \\
\hline 2.2 & $0.677 \pm 0.027$ & $13.3 \pm 0.5$ & $0.961 \pm 0.070$ & $0.533 \pm 0.025$ & $1.54 \pm 0.01$ & $3.11 \pm 0.03$ \\
\hline \multicolumn{7}{|c|}{$\mathrm{G} 1.3(\gamma=1.3)$} \\
\hline 3.0 & $0.612 \pm 0.041$ & $13.6 \pm 0.5$ & $1.044 \pm 0.115$ & $0.633 \pm 0.036$ & $1.71 \pm 0.02$ & $4.22 \pm 0.04$ \\
\hline 2.8 & $0.603 \pm 0.038$ & $14.2 \pm 0.5$ & $0.996 \pm 0.103$ & $0.612 \pm 0.034$ & $1.70 \pm 0.01$ & $4.04 \pm 0.04$ \\
\hline 2.6 & $0.609 \pm 0.033$ & $14.6 \pm 0.6$ & $0.971 \pm 0.089$ & $0.586 \pm 0.031$ & $1.67 \pm 0.01$ & $3.81 \pm 0.03$ \\
\hline 2.4 & $0.625 \pm 0.029$ & $14.8 \pm 0.6$ & $0.951 \pm 0.080$ & $0.551 \pm 0.029$ & $1.64 \pm 0.01$ & $3.53 \pm 0.03$ \\
\hline 2.2 & $0.641 \pm 0.026$ & $14.7 \pm 0.7$ & $0.937 \pm 0.071$ & $0.514 \pm 0.026$ & $1.60 \pm 0.01$ & $3.23 \pm 0.01$ \\
\hline \multicolumn{7}{|c|}{$\mathrm{G} 1.0(\gamma=1.0)$} \\
\hline 3.0 & $0.611 \pm 0.042$ & $13.6 \pm 0.5$ & $1.034 \pm 0.114$ & $0.614 \pm 0.036$ & $1.72 \pm 0.02$ & $4.21 \pm 0.04$ \\
\hline 2.8 & $0.600 \pm 0.037$ & $14.2 \pm 0.5$ & $0.980 \pm 0.100$ & $0.593 \pm 0.034$ & $1.71 \pm 0.02$ & $4.04 \pm 0.04$ \\
\hline 2.6 & $0.605 \pm 0.033$ & $14.7 \pm 0.6$ & $0.949 \pm 0.090$ & $0.565 \pm 0.032$ & $1.68 \pm 0.01$ & $3.82 \pm 0.03$ \\
\hline 2.4 & $0.621 \pm 0.029$ & $14.9 \pm 0.6$ & $0.925 \pm 0.079$ & $0.528 \pm$ & $1.65 \pm 0.01$ & $3.55 \pm 0.02$ \\
\hline 2.2 & $0.638 \pm 0.027$ & $14.9 \pm 0.7$ & $0.909 \pm 0.071$ & $0.490 \pm$ & $1.61 \pm 0.01$ & $3.25 \pm 0.03$ \\
\hline \multicolumn{7}{|c|}{$\operatorname{G1T} 4(\gamma=1.0, \log T=4.0)$} \\
\hline 3.0 & $0.620 \pm 0.043$ & $14.4 \pm 0.5$ & $0.711 \pm 0.087$ & $0.318 \pm 0.039$ & $1.60 \pm 0.02$ & $4.66 \pm 0.06$ \\
\hline 2.8 & $0.594 \pm 0.039$ & $15.5 \pm 0.7$ & $0.683 \pm 0.079$ & $0.317 \pm 0.037$ & $1.60 \pm 0.02$ & $4.53 \pm 0.06$ \\
\hline 2.6 & $0.591 \pm 0.035$ & $16.5 \pm 0.9$ & $0.669 \pm 0.070$ & $0.306 \pm 0.034$ & $1.59 \pm 0.02$ & $4.30 \pm 0.04$ \\
\hline 2.4 & $0.604 \pm 0.020$ & $17.1 \pm 0.9$ & $0.658 \pm 0.044$ & $0.282 \pm 0.020$ & $1.57 \pm 0.02$ & $3.98 \pm 0.04$ \\
\hline 2.2 & $0.621 \pm 0.025$ & $17.4 \pm 1.1$ & $0.651 \pm 0.058$ & $0.254 \pm 0.028$ & $1.54 \pm 0.02$ & $3.61 \pm 0.02$ \\
\hline \multicolumn{7}{|c|}{ S0.76 $(\sigma=0.76)$} \\
\hline 3.0 & $0.762 \pm 0.055$ & $13.7 \pm 0.6$ & $1.383 \pm 0.173$ & $0.788 \pm 0.041$ & $1.66 \pm 0.03$ & $4.58 \pm 0.05$ \\
\hline 2.8 & $0.758 \pm 0.050$ & $14.1 \pm 0.7$ & $1.314 \pm 0.139$ & $0.763 \pm 0.035$ & $1.64 \pm 0.03$ & $4.37 \pm 0.04$ \\
\hline 2.6 & $0.767 \pm 0.045$ & $14.4 \pm 0.6$ & $1.270 \pm 0.122$ & $0.734 \pm 0.032$ & $1.61 \pm 0.02$ & $4.12 \pm 0.04$ \\
\hline 2.4 & $0.783 \pm 0.040$ & $14.6 \pm 0.7$ & $1.242 \pm 0.109$ & $0.700 \pm 0.031$ & $1.58 \pm 0.02$ & $3.83 \pm 0.04$ \\
\hline 2.2 & $0.804 \pm 0.035$ & $14.5 \pm 0.6$ & $1.211 \pm 0.096$ & $0.659 \pm 0.028$ & $1.55 \pm 0.02$ & $3.52 \pm 0.03$ \\
\hline \multicolumn{7}{|c|}{$\mathrm{S} 0.64(\sigma=0.64)$} \\
\hline 3.0 & $0.958 \pm 0.074$ & $13.6 \pm 0.6$ & $2.191 \pm 0.277$ & $0.994 \pm 0.044$ & $1.63 \pm 0.03$ & $5.17 \pm 0.05$ \\
\hline 2.8 & $0.949 \pm 0.069$ & $14.2 \pm 0.5$ & $2.015 \pm 0.238$ & $0.958 \pm 0.041$ & $1.61 \pm 0.02$ & $4.93 \pm 0.04$ \\
\hline 2.6 & $0.958 \pm 0.061$ & $14.7 \pm 0.6$ & $1.879 \pm 0.203$ & $0.915 \pm 0.038$ & $1.59 \pm 0.03$ & $4.67 \pm 0.04$ \\
\hline 2.4 & $0.979 \pm 0.054$ & $15.1 \pm 0.8$ & $1.772 \pm 0.172$ & $0.868 \pm 0.035$ & $1.56 \pm 0.02$ & $4.37 \pm 0.04$ \\
\hline 2.2 & $1.004 \pm 0.051$ & $15.3 \pm 0.8$ & $1.677 \pm 0.151$ & $0.818 \pm 0.032$ & $1.53 \pm 0.02$ & $4.03 \pm 0.04$ \\
\hline
\end{tabular}


Table 10. Bias parameters when varying $\bar{F}$ and $z$ independently.

\begin{tabular}{|c|c|c|c|}
\hline $\mathrm{z}, \bar{F}$ & $\beta$ & $b_{\tau \delta}$ & $b_{\tau \eta}$ \\
\hline \multicolumn{4}{|c|}{ Fiducial } \\
\hline 3.0 & $1.205 \pm 0.049$ & $0.5546 \pm 0.0086$ & $0.681 \pm 0.028$ \\
\hline 2.8 & $1.284 \pm 0.052$ & $0.5577 \pm 0.0083$ & $0.733 \pm 0.030$ \\
\hline 2.6 & $1.343 \pm 0.055$ & $0.5588 \pm 0.0084$ & $0.771 \pm 0.032$ \\
\hline 2.4 & $1.385 \pm 0.056$ & $0.5574 \pm 0.0080$ & $0.796 \pm 0.032$ \\
\hline 2.2 & $1.405 \pm 0.061$ & $0.5536 \pm 0.0079$ & $0.807 \pm 0.034$ \\
\hline
\end{tabular}

\begin{tabular}{c|ccc}
\hline \multicolumn{5}{l}{$\bar{F}$ fixed to 0.781} \\
\hline 3.0 & $1.274 \pm 0.048$ & $0.6038 \pm 0.0061$ & $0.790 \pm 0.021$ \\
2.8 & $1.310 \pm 0.052$ & $0.5813 \pm 0.0068$ & $0.782 \pm 0.026$ \\
2.6 & $1.344 \pm 0.056$ & $0.5588 \pm 0.0077$ & $0.721 \pm 0.031$ \\
2.4 & $1.383 \pm 0.060$ & $0.5356 \pm 0.0093$ & $0.768 \pm 0.039$ \\
2.2 & $1.429 \pm 0.065$ & $0.5121 \pm 0.0110$ & $0.751 \pm 0.052$ \\
\hline$z$ fixed to 2.6 & & \\
\hline 0.696 & $1.275 \pm 0.057$ & $0.517 \pm 0.008$ & $0.676 \pm 0.030$ \\
0.740 & $1.318 \pm 0.058$ & $0.536 \pm 0.008$ & $0.640 \pm 0.036$ \\
0.781 & $1.343 \pm 0.057$ & $0.558 \pm 0.008$ & $0.769 \pm 0.032$ \\
0.818 & $1.347 \pm 0.054$ & $0.581 \pm 0.008$ & $0.804 \pm 0.032$ \\
0.852 & $1.332 \pm 0.069$ & $0.609 \pm 0.008$ & $0.834 \pm 0.032$ \\
\hline \hline
\end{tabular}


Table 11. Non-linear fit parameters when varying $\bar{F}$ and $z$ independently.

\begin{tabular}{c|cccccc}
$\mathrm{z}$ & $q_{1}$ & $k_{p}[h / M p c]$ & $k_{v}^{a_{v}}[h / M p c]^{a_{v}}$ & $a_{v}$ & $b_{v}$ & $k_{n a}$ \\
\hline \hline \multicolumn{7}{l}{ Fiducial } \\
\hline 3.0 & $0.648 \pm 0.042$ & $13.1 \pm 0.4$ & $1.007 \pm 0.103$ & $0.627 \pm 0.033$ & $1.66 \pm 0.01$ & $4.09 \pm 0.04$ \\
2.8 & $0.644 \pm 0.037$ & $13.4 \pm 0.5$ & $0.979 \pm 0.091$ & $0.610 \pm 0.030$ & $1.64 \pm 0.01$ & $3.89 \pm 0.03$ \\
2.6 & $0.652 \pm 0.034$ & $13.6 \pm 0.5$ & $0.970 \pm 0.084$ & $0.590 \pm 0.028$ & $1.61 \pm 0.01$ & $3.65 \pm 0.04$ \\
2.4 & $0.666 \pm 0.030$ & $13.5 \pm 0.5$ & $0.963 \pm 0.076$ & $0.561 \pm 0.027$ & $1.58 \pm 0.01$ & $3.39 \pm 0.03$ \\
2.2 & $0.677 \pm 0.027$ & $13.3 \pm 0.5$ & $0.961 \pm 0.070$ & $0.533 \pm 0.025$ & $1.54 \pm 0.01$ & $3.11 \pm 0.03$ \\
\hline $\bar{F}$ fixed to 0.781 & & & & & \\
\hline 3.0 & $0.710 \pm 0.041$ & $16.0 \pm 0.8$ & $1.195 \pm 0.114$ & $0.703 \pm 0.033$ & $1.66 \pm 0.02$ & $4.12 \pm 0.04$ \\
2.8 & $0.683 \pm 0.036$ & $14.7 \pm 0.7$ & $1.070 \pm 0.093$ & $0.644 \pm 0.029$ & $1.64 \pm 0.02$ & $3.88 \pm 0.04$ \\
2.6 & $0.652 \pm 0.033$ & $13.6 \pm 0.5$ & $0.970 \pm 0.083$ & $0.590 \pm 0.029$ & $1.61 \pm 0.02$ & $3.65 \pm 0.03$ \\
2.4 & $0.620 \pm 0.030$ & $12.5 \pm 0.5$ & $0.876 \pm 0.071$ & $0.530 \pm 0.027$ & $1.58 \pm 0.02$ & $3.43 \pm 0.03$ \\
2.2 & $0.585 \pm 0.028$ & $11.6 \pm 0.4$ & $0.793 \pm 0.064$ & $0.473 \pm 0.026$ & $1.56 \pm 0.02$ & $3.22 \pm 0.04$ \\
\hline$z$ fixed to 2.6 & & & & & \\
\hline
\end{tabular}

